\title{
AdDitions to the floral WEALTH Of SIRMAUR District, HIMACHAL PRADESH FROM CHURDHAR WILDLIFE SANCTUARY
}

\author{
S.P. Subramani ${ }^{1}$, K.S. Kapoor ${ }^{2}$ \& G.S. Goraya ${ }^{3}$ \\ 1,2,3 Himalayan Forest Research Institute (HFRI), Conifer Campus, Panthaghati, Shimla, Himachal Pradesh 171009, India \\ ${ }^{1}$ Present Address: Institute of Forest Genetics and Tree Breeding (IFGTB), Forest Campus, R.S. Puram, Coimbatore, \\ Tamil Nadu 641002, India \\ ${ }^{3}$ Present Address: Deputy Director General, Indian Council of Foretry Research \& Education (ICFRE), Dehra Dun, \\ Uttarkhand 248006, India \\ ${ }^{1}$ subramanisp@icfre.org (corresponding author), ${ }^{2}$ kapoorks@icfre.org, ${ }^{3}$ gurinder9@hotmail.com
}

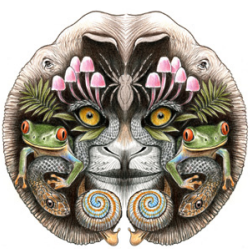

ISSN 0974-7907 (Online) ISSN 0974-7893 (Print)

\section{OPEN ACCESS}

Abstract: A total of 352 species of phenerogams comprising 251 dicotyledons, 97 monocotyledons and four species of gymnosperms belonging to 85 families collected from Nohra Forest Block of Churdhar Wildlife Sanctuary, District Sirmaur, Himachal Pradesh are reported as additions to the district. This includes 13 threatened species under different Red List assessments, both global and regional and 35 species are endemic to western Himalaya.

Keywords: Additions, Churdhar, flora, Himachal Pradesh, protected area, Sirmaur.

Protected areas (PAs) like wildlife reserves and national parks, cover more than $12 \%$ of the Earth's surface and are internationally recognized as a major tool in conservation programmes (Khan \& Bhagwat 2010). They maintain key habitats, provide refugia, allow species migration and movement and provide goods and services otherwise essential for sustainable use of natural resources. As a result, they store more than $15 \%$ of the terrestrial carbon (Holdgate 1999) and also serve as a tool for containing future exceptional loss of habitat. Accordingly, it has now been realized that inventorisation of plants occurring in such areas is absolutely necessary and so are the initiatives from the Ministry of Environment, Forests, \& Climate Change, Government of India, which is now showing great concern for compilation or documentation of floral elements in these areas of importance, ultimately leading towards ensuring the welfare of faunal elements. Though 20177 taxa of flowering plants have been reported from India (Karthikeyan 2009), several areas still remain underexplored and pockets of many forests botanically less studied.

Churdhar Wildlife Sanctuary (CWS) is one of the few remaining citadels of biodiversity that covers wide

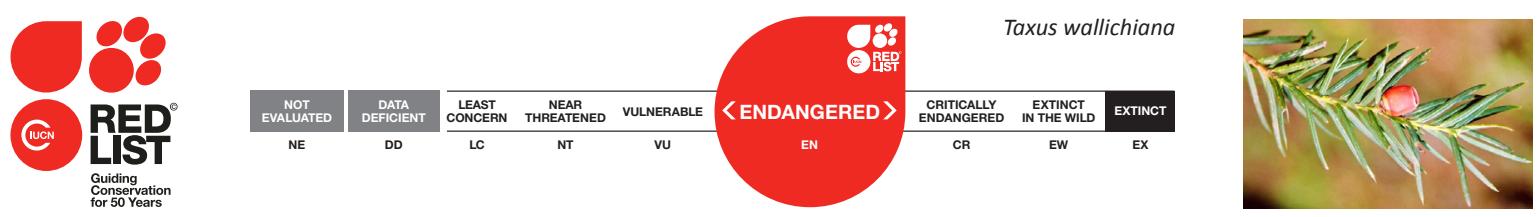

DOI: http://dx.doi.org/10.11609/JoTT.02845.6427-52

Editor: K. Ravikumar, FRLHT, Bengaluru, India.

Date of publication: 26 October 2014 (online \& print)

Manuscript details: Ms \# 02845 | Received 24 June 2011 | Final received 01 September 2014 | Finally accepted 23 September 2014

Citation: Subramani, S.P., K.S. Kapoor \& G.S. Goraya (2014). Additions to the floral wealth of Sirmaur District, Himachal Pradesh from Churdhar Wildlife Sanctuary. Journal of Threatened Taxa 6(11): 6427-6452; http://dx.doi.org/10.11609/JoTT.02845.6427-52

Copyright: @ Subramani et al. 2014. Creative Commons Attribution 4.0 International License. JoTT allows unrestricted use of this article in any medium, reproduction and distribution by providing adequate credit to the authors and the source of publication.

Funding: Self funded.

Competing Interest: The authors declare no competing interests.

Acknowledgements: The authors are thankful to the Director, Himalayan Forest Research Institute, Shimla for allowing us to use the related facilities and for encouragement during the study. Besides, we express our gratitude to Shri Vinay Tandon, IFS, PCCF Himachal Pradesh State Forest Department, Shimla for granting permission. We are also thankful to the Head, Botany Division FRI, Dehra Dun for providing all facilities at DD Herbarium, Dehra Dun during the matching of the voucher specimens. Help rendered by Dr. Vaneet Jishtu, Sh. Purushotham Ram and Dushyant Kumar at different stages of the study is thankfully acknowledged. 
altitudinal ranges where documentation is certainly inadequate. However, some parts of this sanctuary face heavy threats from a comparatively high density of livestock population belonging to the Gujjar community, residing in the area alongwith their herds for grazing purposes since long. Besides, visits of thousands and thousands of pilgrims annually to Chooreshwar Mahadev Temple at Chur peak-a religiously important placeis adding towards the voes since populations of a few species like Angelica glauca, Betula utilis, Cypripedium cordigerum, C. himalaicum, Dactylorhiza hatagirea, Rhododendron anthopogon, R. campanulatum, Taxus wallichaiana and Valeriana jatamansi etc. are in rapid decline during the recent decades.

Botanical explorations of Royle in 1823, Drummond during 1883-1885 and Clarke in 1884, as carried out in the past are some of the pioneering works from Chur Mountains. During the $19^{\text {th }}$ century Collett (1902), and Nair (1977) also made pilot surveys in this area. However, these two studies covered only the Pulbahal Forest Block of the sanctuary falling in Shimla District, Himachal Pradesh. Studies undertaken here in the recent past include the works of Gupta (1998) and Kumar (2004) but they recorded only the medicinal flora of the sanctuary. The first published account on the flora of Sirmaur District is of Kaur \& Sharma (2004), wherein they reported 911 species belonging to 544 genera under 139 families again only touching the boundaries of the sanctuary and its core area.

The present study had been carried out systematically for assessing the floristic wealth of Churdhar Wildlife Sanctuary including its rich potential of herbal medicinal plants. However, in this paper only Nohra Block of the sanctuary and the additions to the flora of district Sirmaur have been highlighted.

\section{STUDY AREA}

Churdhar Wildlife Sanctuary-one of the important protected areas of the Himalayan region-is located in the Trans-Giri hill ranges of Sirmaur and Shimla districts in the state of Himachal Pradesh. The sanctuary which falls around Chur Peak $(3,647 \mathrm{~m})$, one of the Shivalik's highest summits in sub-tropical Himalaya, was established in the year 1985 with a view to preserve the biological wealth and floral diversity in the Panchmuda Range of Churdhar. The sanctuary comprises an approximate area of $66.70 \mathrm{~km}^{2}$ with wide altitudinal ranges varying from approximately $1900-3647 m$ and lies in between $30^{\circ} 48^{\prime} 37^{\prime \prime}-30^{\circ} 54^{\prime} 39^{\prime \prime} \mathrm{N} \& 77^{\circ} 23^{\prime} 32^{\prime \prime}-$ $77^{\circ} 29^{\prime} 49^{\prime \prime} \mathrm{E}$. From a management point of view, the sanctuary area has been divided into two forest blocks namely, Nohra and Pulbahal. Approximately, 65\% of the study area falls in Nohra Block that has three forest beats, i.e., Nohra, Churas and Chhog Thali falling in Sirmaur District (Fig. 1) whereas the remaining 35\% of the sanctuary area is in Pulbahal Block which has two forest beats namely Sarpat and Bharog and forms a part of district Shimla (Anonymous 1994-1995; Anonymous 2005-2006). Based on the altitude, aspect and climatic conditions, the sanctuary harbours a wide array of vegetation types ranging from anthropogenic grasslands in lower hill ranges to alpine meadows in the mountain peaks whereas the oak forests, moist deodar forests and western mixed conifer forests occupy the major portion of the middle hill ranges. Because of its rich floral elements in distinct altitudinal zonations including good faunal wealth, the area is spectacular and is quite important for detailed investigations.

\section{MATERIAL AND MethodS}

For complete botanical explorations and phytosociological studies for quantification of the floristic elements in different altitudinal zonations of the sanctuary, periodic field trips were undertaken starting

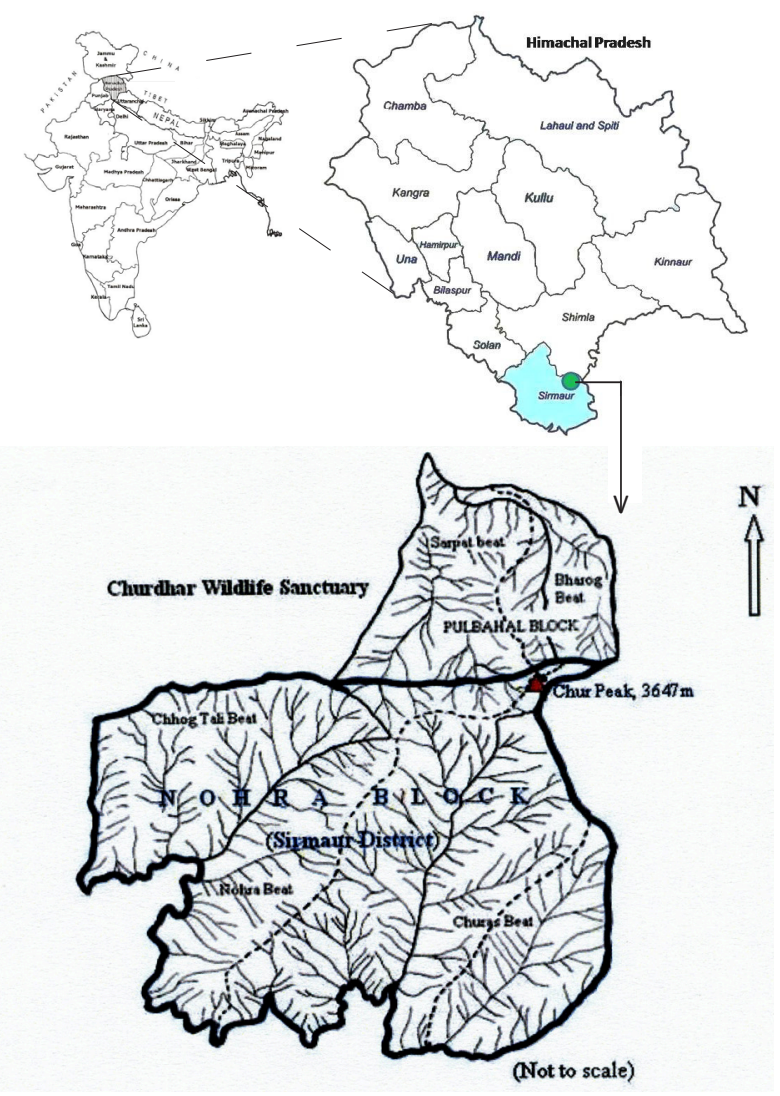

Figure 1. Churdhar Wildlife Sanctuary showing location of Nohra Block, Sirmaur District, Himachal Pradesh 
from March 2002 till September 2008 in different forest types including macro- and micro-habitats. The voucher specimens thus collected, were identified using local Floras (Chowdhery \& Wadhwa 1984; Polunin \& Stainton 1984; Collett 1902; Kaur \& Sharma 2004) and species indentity was further confirmed by comparing the authentic specimens housed in DD Herbarium of Forest Research Institute and Herbarium of Botanical Survey of India, Dehra Dun. Families of the specimens are arranged following Bentham and Hooker's classification and under each family, species were arranged alphabetically with their recent botanical name, habit, flowering season, forest area and altitudinal zonations. During the process of verification of herbarium specimens and their screening with existing literature for their earlier records many taxa collected from Nohra Block of Sirmaur area as highlighted in the present attempt, turned out to be new additions to the flora of district Sirmaur, Himachal Pradesh. Voucher specimens for each species are also deposited in the herbarium of Himalayan Forest Research Institute (HFRI), Shimla.

\section{RESULTS AND DISCUSSIONS}

A total of 793 taxa with 748 angiosperms, eight gymnosperms and 37 pteridophytes belonging to 431 genera distributed under 143 families were collected during the survey period. A further scrutiny of the literature revealed that out of the total 756 species of phenerogams, 352 species representing 251 dicotyledons, 97 monocotyledons and four species of gymnosperms spread over to 85 families were hitherto unreported from district Sirmaur, Himachal Pradesh (Table 1). In this paper the floral elements from Nohra Block of CWS has been listed which forms new records for Sirmaur District, Himachal Pradesh. Kaur \& Sharma (2004) in their earlier studies had documented only 911 taxa from district Sirmaur having a total geographical area of $2,825 \mathrm{~km}^{2}$, whereas, the outcome of the present intensive botanical investigation in CWS alone has yielded 756 taxa of flowering plants and conifers within the $66.70 \mathrm{~km}^{2}$ area of the sanctuary. It clearly reflects that this particular sanctuary is the citadel of plant diversity and accordingly, a number of plant lovers in the past, appreciated its ecological importance and subsequently developed an interest in this area, though they did not have much of the scientific data to explain its relevance. Hence, the present study besides, providing the firm scientific footing to the earlier accounts, has further pointed out that the focused approach for the management of the sanctuary is essentially required from its conservation point of view.
In this enumeration as carried out in the sanctuary, the dominant 10 families are Poaceae followed by Asteraceae, Orchidaceae, Cyperaceae, Ranunculaceae, Scrophulariaceae, Apiaceae, Papilionoideae, Rosaceae and Caprifoliaceae which contribute $45 \%$ of the total collections (Fig. 2). Though the habit of plants are exhibited in all life forms, herbaceous habit was found to be the most dominant among others, followed by shrubs, trees, climbers and others as shown in Fig. 3.

Out of the total 352 species, 35 taxa, viz., Acer acuminatum, A. caesium, Aconitum laeve, Aconogonum rumicifolium, Arenaria festucoides, Bupleurum lanceolatum, Buxus wallichiana, Chaerophyllum reflexum var. acuminatum, Clematis barbellata, Cortusa brotheri, Corylus jacquemontii, Delphinium vestitum, Hedysarum microcalyx, Impatiens glauca, Jaeschkea canaliculata, Lindelofia longiflora, Meconopsis aculeata, Nepeta connata, Pedicularis bicornuta, P. hoffmeisteri, P. pectinata, P. pyramidata, Pleurospermum brunonis, P. candollei, Polygonum paronychioides, Primula reidii, Rhamnus procumbens, R. purpurea, Salix denticulata, Selinum vaginatum, Silene indica var. edgeworthii, $S$. laxantha, Syringa emodi, Trollius acaulis and Wulfeniopsis amherstiana are endemic to western Himalaya, one of the mega endemic centres in India (Nayar 1996). Acer caesium, Cypripedium cordigerum, C. himalaicum, Dioscorea deltoidea and Hedysarum microcalyx included in the Red Data Book of Indian Plants (Nayar \& Sastry $1987,1990)$, were also collected from the study area. The images of live plants of some important species are shown in Images 1-55.

Dactylorhiza hatagirea and Malaxis muscifera (Critically Endangered); Angelica glauca, Betula utilis, Meconopsis aculeata, Paris polyphylla and Polygonatum cirrhifolium (Endangered); Polygonatum verticillatum, Rhodiola heterodonta, Rhododendron anthopogon, and $R$. lepidotum (Vulnerable) were also located in the study area as assessed regionally for Himachal Pradesh (Ved et al. 2003). Taxus wallichiana is assessed as Endangered and Abies spectabilis is assessed as Near Threatened by the IUCN Red List of Threatened Species (Thomas \& Farjon 2011).

Though Churdhar Wildlife Sanctuary is a small study area when compared to the total geo-graphical area of Sirmaur District, it is certainly a potential hunting ground for continued floristic investigations so as to unearth new species and new distribution records. A knowledge of its flora and fauna is certainly of utmost significance in addition to other aspects of ecosystem functioning for better management of this protected area. This study also indicates the positive response of in situ 


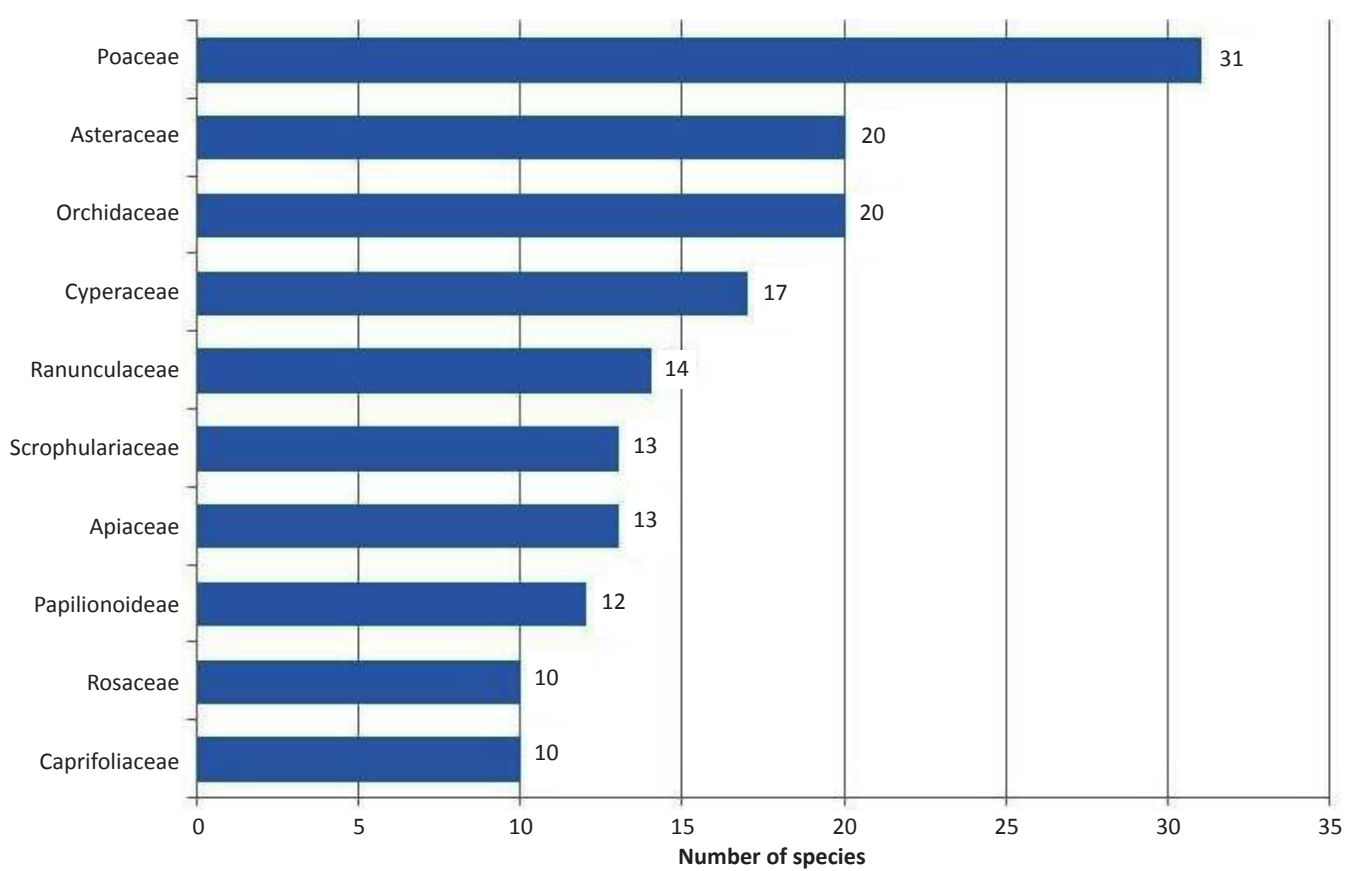

Figure 2. Ten dominant families with respect to species of the additions to the flora of Sirmaur District, Himachal Prades

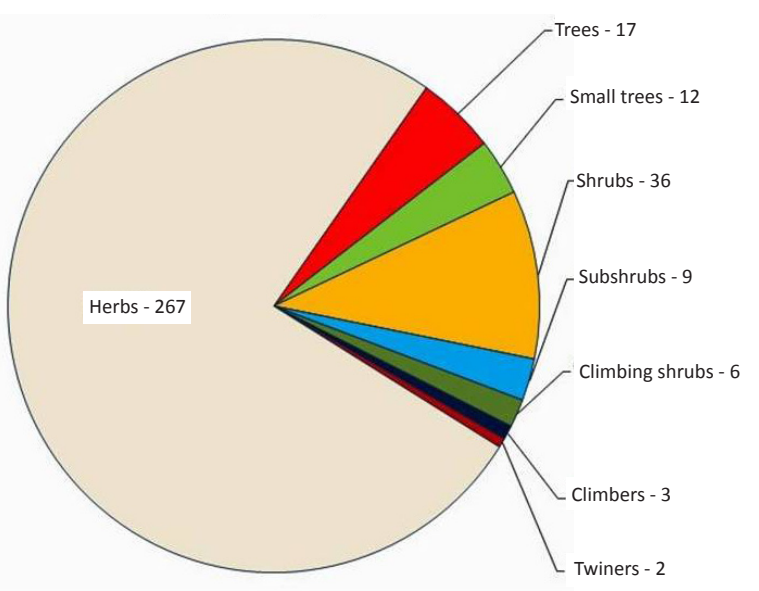

Figure 3. Habit wise break up of the addtions to the flora of Sirmaur District, Himachal Prades (352 taxa)

conservation of ecosystems and natural habitats under protected area networking (Singh 2002) in Convention on Biological Diversity's (CBD) Article 8 which is one of the most widely accepted and practically approachable strategies to biodiversity conservation.

\section{REFERENCES}

Anonymous (1994-95). Revised Working Plan for the Forests of Chopal Forest Division, Himachal Pradesh. Vol.1: 356pp.

Anonymous (2005-06). Revised Working Plan for the Forests of
Rajgarh Forest Division, Himachal Pradesh. Vol.1: 387pp.

Chowdhery, H.J. \& B.M. Wadhwa (1984). Flora of Himachal Pradesh, Analysis Vols. 1-3. Botanical Survey of India, Calcutta, 860pp.

Collett, H. (1902). Flora Simlensis: A Handbook of the Flowering Plants of Simla and the Neighbourhood. Thacker Spink and Co., Calcutta, $652 \mathrm{pp}$.

Gupta, H. (1998). Comparative studies on the medicinal and aromatic flora of Churdhar and Rohtang areas of Himachal Pradesh. MSc Thesis. Dr. Y.S. Parmar University of Horticulture and Forestry, Nauni, Solan (unpuplished).

Holdgate, M. (1999). The Green Web - A Union for World Conservation. Earthscan, London, UK.

Karthikeyan, S. (2009). Flowering Plants of India in $19^{\text {th }}$ and $21^{\text {st }}$ Centuries - comparison, pp. 19-30. In: Krishnan, S. \& D. Bhat (eds). Plant and Fungal Biodiversity and Bioprospecting. Boradway Book Centre, Goa.

Kaur, H. \& M. Sharma (2004). Flora of Sirmaur (Himachal Pradesh). Bishen Singh Mahendra Pal Singh, Dehra Dun, 770 pp.

Khan, M.S. \& S.A. Bhagwat (2010). Protected areas: a resource or constraint for local people? - A study at Chitral Gol National Park, North-West Frontier Province, Pakistan. Mountain Research and Development 30(1): 14-24.

Kumar, N. (2004). Studies on the distribution and importance of medicinal and aromatic plants of Nahan area, District Sirmaur (H.P.). MSc Thesis. Dr. Y.S. Parmar University of Horticulture and Forestry, Nauni, Solan (unpublished), 226pp.

Nair, N.C. (1977). Flora of Bashahr Himalaya. International Bioscience Publishers, Hissar, 360pp.

Nayar, M.P. (1996). Hot Spots of Endemic Plants of India, Nepal and Bhutan. Tropical Botanic Garden and Research Institute, Palode, Thiruvananthapuram, 252pp.

Nayar, M.P. \& A.R.K. Sastry (eds) $(1987,1990)$. Red Data Book of Indian Plants. Vol.1 - (1987) \& Vol. 3 - (1990). Botanical Survey of India, Howrah.

Polunin, O. \& A. Stainton (1984). Flowers of the Himalaya. Oxford University Press, New Delhi, 580pp.

Singh, J.S. (2002). The biodiversity crisis: A multifaceted review. Current Science 82: 638-647. 
Table 1. List of the plant species which are additions to the Flora of Sirmaur District, Himachal Pradesh.

\begin{tabular}{|c|c|c|c|c|c|c|c|}
\hline & Botanical Name & Habit & $\begin{array}{l}\text { HFRI } \\
\text { collection } \\
\text { no. }\end{array}$ & $\begin{array}{l}\text { Flowering \& } \\
\text { fruiting }\end{array}$ & $\begin{array}{l}\text { Altitudinal } \\
\text { range }(\mathrm{m})\end{array}$ & Forest area & Remarks \\
\hline \multicolumn{8}{|c|}{ Dicotyledons } \\
\hline \multicolumn{8}{|c|}{ Ranunculaceae } \\
\hline 1 & Aconitum laeve Royle & $\mathrm{H}$ & SPS 3526 & July-Sept. & 2950-3000 & CT & $\begin{array}{l}\text { Rare, in humus rich soil, under Fir, } \\
\text { Oak forests }\end{array}$ \\
\hline 2 & $\begin{array}{l}\text { Actaea spicata L. var. acuminata } \\
\text { (Wall. ex Royle) H. Hara }\end{array}$ & $\mathrm{H}$ & SPS 4173 & June-Sept. & $3200-3600$ & $\mathrm{~N}, \mathrm{C}$ & Rare, in subalpine meadows \\
\hline 3 & Aquilegia pubiflora Wall. ex Royle & $\mathrm{H}$ & $\begin{array}{l}\text { SPS } 3583 \\
3675,3779\end{array}$ & May-July & $2900-3200$ & CT & Occasional in mixed conifer forests \\
\hline 4 & Clematis barbellata Edgew. & CS & SPS 4151 & April-July & $2600-3100$ & $\mathrm{~N}$ & Occasional along waysides \\
\hline 5 & Delphinium vestitum Wall. ex Royle & $\mathrm{H}$ & $\begin{array}{l}\text { SPS 3960, } \\
4042\end{array}$ & June-Sept. & $2800-3600$ & $\mathrm{~N}$ & Frequent, in subalpine meadows \\
\hline 6 & Ranunculus adoxifolius Hand.-Mazz. & $\mathrm{H}$ & SPS 3841 & May-Aug. & $3000-3450$ & $\mathrm{~N}, \mathrm{CT}$ & $\begin{array}{l}\text { Occasional, in damp places of alpine } \\
\text { and subalpines }\end{array}$ \\
\hline 7 & Ranunculus pulchellus C.A. Mey. & $\mathrm{H}$ & SPS 4383 & May-July & $2900-3450$ & $\mathrm{~N}$ & $\begin{array}{l}\text { Common, in wet and marshy } \\
\text { habitats }\end{array}$ \\
\hline 8 & $\begin{array}{l}\text { Ranunculus trichophyllus Chaix ex } \\
\text { Vill. }\end{array}$ & $\mathrm{H}$ & SPS 4301 & May-Sep. & $2300-2600$ & C & Rare, in marshy habitats \\
\hline 9 & Thalictrum alpinum $\mathrm{L}$. & $\mathrm{H}$ & $\begin{array}{l}\text { SPS 3708, } \\
4007\end{array}$ & June-July & $3150-3550$ & $\mathrm{~N}, \mathrm{C}$ & $\begin{array}{l}\text { Frequent, in alpines below Chur } \\
\text { peak }\end{array}$ \\
\hline 10 & Thalictrum cultratum Wall. & $\mathrm{H}$ & SPS 3858 & June-Sep. & $3000-3640$ & $\mathrm{~N}$ & $\begin{array}{l}\text { Common, among boulders below } \\
\text { Chur peak }\end{array}$ \\
\hline 11 & Thalictrum minus $\mathrm{L}$. & H & SPS 3723 & May-July & $2800-3100$ & C & $\begin{array}{l}\text { Rare, among rocky crevices, Churas } \\
\text { beat }\end{array}$ \\
\hline 12 & Thalictrum pedunculatum Edgew. & $\mathrm{H}$ & SPS 3574 & April-Aug. & $2900-3400$ & $\mathrm{~N}$ & $\begin{array}{l}\text { Rare, among boulders, below Chur } \\
\text { peak }\end{array}$ \\
\hline 13 & Thalictrum reniforme Wall. & $\mathrm{H}$ & $\begin{array}{l}\text { SPS 4052, } \\
4302\end{array}$ & June-Oct. & $2600-3150$ & $\mathrm{~N}, \mathrm{CT}$ & Rare, inhabits damp places \\
\hline 14 & Trollius acaulis Lindl. & H & SPS 3630 & June-Aug. & $3300-3600$ & CT & Rare, in alpine slopes \\
\hline \multicolumn{8}{|c|}{ Berberidaceae } \\
\hline 15 & Berberis aristata DC. & S & SPS 4021 & April-May & $2000-2600$ & C & $\begin{array}{l}\text { Occasional, in the outskirts of } \\
\text { forests }\end{array}$ \\
\hline 16 & Berberis coriaria Royle ex Lindl. & S & SPS 3518 & April-June & $1900-2400$ & CT & $\begin{array}{l}\text { Occasional, in the outskirts of } \\
\text { forests }\end{array}$ \\
\hline 17 & Berberis jaeschkeana C.K. Schneid. & S & $\begin{array}{l}\text { SPS } 3617, \\
3714\end{array}$ & May-July & $2900-3400$ & $\mathrm{~N}, \mathrm{C}$ & $\begin{array}{l}\text { Frequent, above timber-line and in } \\
\text { sub-alpine scrub }\end{array}$ \\
\hline \multicolumn{8}{|c|}{ Papaveraceae } \\
\hline 18 & Meconopsis aculeata Royle & H & $\begin{array}{l}\text { SPS } 3695 \\
3727\end{array}$ & June-Sep. & $2900-3400$ & $\mathrm{~N}, \mathrm{C}$ & Rare, found in moist, rock crevices \\
\hline \multicolumn{8}{|c|}{ Fumariaceae } \\
\hline 19 & Corydalis vaginans Royle & $\mathrm{H}$ & $\begin{array}{l}\text { SPS 3752, } \\
3974\end{array}$ & April-June & $3200-3500$ & $\mathrm{C}, \mathrm{CT}$ & Occasional, in subalpine meadows \\
\hline \multicolumn{8}{|c|}{ Brassicaceae } \\
\hline 20 & Arabis pterosperma Edgew. & H & SPS 4435 & Mar.-June & 2900-3300 & $\mathrm{N}, \mathrm{CT}$ & Occasional, in moist localities \\
\hline 21 & Cardamine macrophylla Willd. & $\mathrm{H}$ & SPS 3872 & May-July & $2900-3300$ & $\mathrm{~N}$ & Rare, in moist places, stony slopes \\
\hline 22 & $\begin{array}{l}\text { Crucihimalaya stricta (Cambess.) Al- } \\
\text { Shehbaz, O'Kane \& R.A. Prince }\end{array}$ & H & SPS 3847 & June-July & $1500-3000$ & $\mathrm{~N}$ & $\begin{array}{l}\text { Frequent, in shady places along } \\
\text { waysides }\end{array}$ \\
\hline 23 & Thlaspi arvense $\mathrm{L}$. & H & SPS 4305 & April-July & $2600-2900$ & CT & $\begin{array}{l}\text { Occasional, in exposed slopes, } \\
\text { outskirts of forests }\end{array}$ \\
\hline 24 & Thlaspi cochleariforme DC. & $\mathrm{H}$ & SPS 4033 & June-Aug. & $2900-3200$ & C & $\begin{array}{l}\text { Occasional, under Kharshu oak } \\
\text { forests }\end{array}$ \\
\hline \multicolumn{8}{|c|}{ Violaceae } \\
\hline 25 & Viola betonicifolia Sm. & $\mathrm{H}$ & SPS 4009 & July-Aug. & $2650-2900$ & $\mathrm{~N}$ & Rare, in exposed stony slopes \\
\hline 26 & Viola pilosa Blume & H & SPS 3666 & April-Sep. & $2800-3050$ & $\mathrm{~N}$ & $\begin{array}{l}\text { Occasional, in shady places, stony } \\
\text { slopes }\end{array}$ \\
\hline \multicolumn{8}{|c|}{ Polygalaceae } \\
\hline 27 & $\begin{array}{l}\text { Polygala crotalarioides Buch.-Ham. } \\
\text { ex DC. }\end{array}$ & $\mathrm{H}$ & SPS 4446 & July-Sep. & $1900-2200$ & C & $\begin{array}{l}\text { Common, in grassy slopes, and in } \\
\text { foothills }\end{array}$ \\
\hline 28 & Polygala hottentotta C.PresI. & $\mathrm{H}$ & SPS 3903 & May-Sep. & $2100-2300$ & CT & Occasional, in exposed stony slopes \\
\hline
\end{tabular}




\begin{tabular}{|c|c|c|c|c|c|c|c|}
\hline & Botanical Name & Habit & $\begin{array}{l}\text { HFRI } \\
\text { collection } \\
\text { no. }\end{array}$ & $\begin{array}{l}\text { Flowering \& } \\
\text { fruiting }\end{array}$ & $\begin{array}{l}\text { Altitudinal } \\
\text { range }(\mathrm{m})\end{array}$ & Forest area & Remarks \\
\hline \multicolumn{8}{|c|}{ Caryophyllaceae } \\
\hline 29 & Arenaria festucoides Benth. & $\mathrm{H}$ & SPS 3533 & July-Sep. & $3150-3450$ & $N$ & $\begin{array}{l}\text { Occasional, in dripping slopes at } \\
\text { alpines meadows }\end{array}$ \\
\hline 30 & $\begin{array}{l}\text { Lepyrodiclis holosteoides (C.A. Mey.) } \\
\text { Fenzl ex Fisch. \& C.A. Mey. }\end{array}$ & $\mathrm{H}$ & SPS 3746 & June-July & $3000-3400$ & $N$ & $\begin{array}{l}\text { Frequent, moist, shady places in } \\
\text { subalpine scrub }\end{array}$ \\
\hline 31 & Myosoton aquaticum (L.) Moench & $\mathrm{H}$ & SPS 4503 & May-Oct. & $2200-3200$ & $N$ & Common, in moist, shady localities \\
\hline 32 & Silene indica (Roxb.) Roxb. ex Otth & $\mathrm{H}$ & $\begin{array}{l}\text { SPS 3722, } \\
3627\end{array}$ & July-Oct. & $2900-3250$ & CT & $\begin{array}{l}\text { Frequent, under shrubberies of } \\
\text { temperate forests }\end{array}$ \\
\hline 33 & $\begin{array}{l}\text { Silene indica (Roxb.) Roxb. ex Otth } \\
\text { var. edgeworthii (Bacquet) Y.J. Nasir }\end{array}$ & $\mathrm{H}$ & SPS 3783 & Aug.-Oct. & 2100-3000 & $N$ & $\begin{array}{l}\text { Frequent, under shrubberies of } \\
\text { Deodar forests }\end{array}$ \\
\hline 34 & Silene laxantha Majumdar & $\mathrm{H}$ & $\begin{array}{l}\text { SPS 3778, } \\
3862\end{array}$ & June-Aug. & $3000-3250$ & $N$ & Rare, under Rhododendron forests \\
\hline 35 & Silene viscosa (L.) Pers. & $\mathrm{H}$ & SPS 3782 & May-Aug. & $3050-3300$ & CT & Frequent, in subalpine slopes \\
\hline 36 & Silene vulgaris (Moench) Garcke & $\mathrm{H}$ & SPS 3674 & April-Aug. & $2800-3050$ & $\mathrm{~N}, \mathrm{CT}$ & $\begin{array}{l}\text { Occasional, in exposed slopes, } \\
\text { shady places }\end{array}$ \\
\hline 37 & $\begin{array}{l}\text { Stellaria monosperma Buch.-Ham. } \\
\text { ex D. Don }\end{array}$ & $\mathrm{H}$ & SPS 3784 & July-Aug. & $2800-3100$ & $N$ & $\begin{array}{l}\text { Frequent, in humus covered } \\
\text { grounds under conifer forests }\end{array}$ \\
\hline \multicolumn{8}{|c|}{ Hypericaceae } \\
\hline 38 & $\begin{array}{l}\text { Hypericum choisianum Wall. ex } \mathrm{N} \text {. } \\
\text { Robson }\end{array}$ & $\mathrm{s}$ & SPS 3957 & June-Sep. & $2400-2900$ & $\mathrm{~N}, \mathrm{C}$ & Rare, in outskirts of forests \\
\hline 39 & Hypericum dyeri Rehder & SS & SPS 3504 & April-Aug. & $2200-2450$ & CT & Rare, in deep slopes, rocky places \\
\hline 40 & Hypericum perforatum $\mathrm{L}$. & $\mathrm{H}$ & SPS 3997 & April-Sep. & $2100-2500$ & CT & $\begin{array}{l}\text { Rare, in shady places under } \\
\text { shrubberies }\end{array}$ \\
\hline \multicolumn{8}{|c|}{ Malvaceae } \\
\hline 41 & Malva neglecta Wallr. & $\mathrm{H}$ & SPS 4542 & May-Sep. & $2200-3100$ & CT & $\begin{array}{l}\text { Frequent, in waysides, heavy grazed } \\
\text { forest areas }\end{array}$ \\
\hline \multicolumn{8}{|c|}{ Geraniaceae } \\
\hline 42 & Erodium cicutarium (L.) L' Her. & $\mathrm{H}$ & SPS 4561 & July-Aug. & $3000-3200$ & C & Occasional, in damp places \\
\hline 43 & Geranium robertianum L. & $\mathrm{H}$ & SPS 4512 & June-Sep. & $2200-2450$ & C, CT & Occasional, in moist slopes \\
\hline \multicolumn{8}{|c|}{ Balsaminaceae } \\
\hline 44 & Impatiens amplexicaulis Edgew. & $\mathrm{H}$ & $\begin{array}{l}\text { SPS 3834, } \\
3956 \\
\end{array}$ & July-Sep. & $2900-3200$ & $N$ & $\begin{array}{l}\text { Frequent, a moisture loving plant, } \\
\text { on dripping slopes }\end{array}$ \\
\hline 45 & $\begin{array}{l}\text { Impatiens arguta Hook.f. \& } \\
\text { Thomson }\end{array}$ & $\mathrm{H}$ & SPS 4069 & July-Sep. & $2600-3100$ & $\mathrm{~N}, \mathrm{CT}$ & $\begin{array}{l}\text { Occasional, in shady places and on } \\
\text { decayed woods }\end{array}$ \\
\hline 46 & Impatiens brachycentra Kar. \& Kir. & $\mathrm{H}$ & SPS 3895 & Aug.-Sep. & $1900-2100$ & $\mathrm{~N}$ & Common, along water channels \\
\hline 47 & Impatiens glandulifera Royle & $\mathrm{H}$ & SPS 3973 & July-Sep. & $2400-3100$ & $\mathrm{~N}, \mathrm{CT}$ & $\begin{array}{l}\text { Common, along water channels, } \\
\text { damp places }\end{array}$ \\
\hline 48 & $\begin{array}{l}\text { Impatiens glauca Hook.f. \& } \\
\text { Thomson }\end{array}$ & $\mathrm{H}$ & SPS 3534 & July-Aug. & $3200-3350$ & C & $\begin{array}{l}\text { Rare, recorded only few individuals } \\
\text { at dry alpine slopes }\end{array}$ \\
\hline 49 & Impatiens laxiflora Edgew. & $\mathrm{H}$ & SPS 3587 & June-Sep. & $2800-3200$ & $N$ & Frequent, in moist, shady places \\
\hline 50 & Impatiens racemosa DC. & $\mathrm{H}$ & SPS 4514 & July-Oct. & 2500-3000 & $\mathrm{N}$ & Frequent, in moist, shady places \\
\hline 51 & Impatiens sulcata Wall. & $\mathrm{H}$ & SPS 3800 & July-Sep. & $2700-3100$ & $N$ & Occasional, in moist places \\
\hline \multicolumn{8}{|c|}{ Rutaceae } \\
\hline 52 & $\begin{array}{l}\text { Skimmia laureola (DC.) Sieb. \& Zucc. } \\
\text { ex Walp. }\end{array}$ & 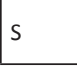 & $\begin{array}{l}\text { SPS 4022, } \\
4311\end{array}$ & April-June & $2600-3100$ & $\mathrm{~N}, \mathrm{C}, \mathrm{CT}$ & Rare, under conifer forests \\
\hline \multicolumn{8}{|c|}{ Simaroubaceae } \\
\hline 53 & Ailanthus altissima (Mill.) Swingle & ST & SPS 4511 & May-Sep. & $1900-2200$ & C, CT & Occasional, along nallahs \\
\hline \multicolumn{8}{|c|}{ Celastraceae } \\
\hline 54 & Euonymus echinatus Wall. & CS & SPS 3928 & April-Oct. & $2400-3450$ & c & $\begin{array}{l}\text { Frequent, creeping over dead trees, } \\
\text { moist rocks }\end{array}$ \\
\hline 55 & Euonymus hamiltonianus Wall. & ST & $\begin{array}{l}\text { SPS 3877, } \\
3942,4010\end{array}$ & June-Sep. & $2900-3200$ & $\mathrm{~N}, \mathrm{CT}$ & Occasional, under conifer forests \\
\hline 56 & Euonymus lucidus D. Don & $\mathrm{T}$ & SPS 3901 & April-July & $2600-3000$ & $\mathrm{~N}$ & Occasional, under Oak forests \\
\hline \multicolumn{8}{|c|}{ Rhamnaceae } \\
\hline 57 & Rhamnus procumbens Edgew. & s & SPS 3642 & May-Oct. & $2400-2650$ & C, CT & Occasional, on exposed rocky slopes \\
\hline 58 & Rhamnus purpurea Edgew. & $\mathrm{s}$ & $\begin{array}{l}\text { SPS 3532, } \\
3656,4008\end{array}$ & May-July & $1900-2200$ & $N$ & Rare, under Oak forests \\
\hline
\end{tabular}




\begin{tabular}{|c|c|c|c|c|c|c|c|}
\hline & Botanical Name & Habit & $\begin{array}{l}\text { HFRI } \\
\text { collection } \\
\text { no. }\end{array}$ & $\begin{array}{l}\text { Flowering \& } \\
\text { fruiting }\end{array}$ & $\begin{array}{l}\text { Altitudinal } \\
\text { range }(m)\end{array}$ & Forest area & Remarks \\
\hline \multicolumn{8}{|c|}{ Vitaceae } \\
\hline 59 & $\begin{array}{l}\text { Tetrastigma serrulatum (Roxb.) } \\
\text { Planch. }\end{array}$ & CS & SPS 4441 & Aug.-Nov. & $1900-2150$ & C & $\begin{array}{l}\text { Frequent, spreading over on moist } \\
\text { rocks in slopes }\end{array}$ \\
\hline \multicolumn{8}{|c|}{ Leeaceae } \\
\hline 60 & Leea asiatica (L.) Ridsdale & D & SPS 4318 & July-Dec. & 1900-2000 & C & $\begin{array}{l}\text { Occasional, foothills along water- } \\
\text { bodies }\end{array}$ \\
\hline \multicolumn{8}{|c|}{ Hippocastanaceae } \\
\hline 61 & $\begin{array}{l}\text { Aesculus indica (Wall. ex Cambess.) } \\
\text { Hook. }\end{array}$ & $\mathrm{T}$ & SPS 4088 & June-Sep. & $2650-2900$ & CT & Rare, along water channels \\
\hline \multicolumn{8}{|c|}{ Aceraceae } \\
\hline 62 & Acer acuminatum Wall. ex D. Don & ST & SPS 4029 & Feb.-May & $2450-2900$ & $\mathrm{~N}, \mathrm{C}$ & Rare, under conifer forests \\
\hline 63 & Acer caesium Wall. ex Brandis & $\mathrm{T}$ & SPS 3915 & Mar.-Oct. & $2650-3150$ & $\mathrm{~N}$ & Rare, under Oak forests \\
\hline 64 & Acer cappadocicum Gled. & ST & SPS 4030 & Mar.-June & $2700-3200$ & C & Rare, in open hilly slopes \\
\hline 65 & $\begin{array}{l}\text { Acer pentapomicum Stewart ex } \\
\text { Brandis }\end{array}$ & $\mathrm{T}$ & $\begin{array}{l}\text { SPS 3659, } \\
3869\end{array}$ & April-July & $2650-2900$ & $\mathrm{~N}$ & Rare, under Oak forests \\
\hline \multicolumn{8}{|c|}{ Staphyleaceae } \\
\hline 66 & Staphylea emodi Wall. & $\mathrm{S}$ & SPS 3581 & April-July & $2900-3200$ & $\mathrm{~N}$ & $\begin{array}{l}\text { Occasional, associated with } \\
\text { Rhododendron campanulatum and } \\
\text { Kharshu oaks }\end{array}$ \\
\hline \multicolumn{8}{|c|}{ Anacardiaceae } \\
\hline 67 & $\begin{array}{l}\text { Toxicodendron wallichii (Hook. f.) } \\
\text { Kuntze }\end{array}$ & $\mathrm{T}$ & SPS 4549 & June-Oct. & $1900-2150$ & C & $\begin{array}{l}\text { Occasional, in conifers and broad } \\
\text { leaved mixed forests }\end{array}$ \\
\hline \multicolumn{8}{|c|}{ Papilionoideae } \\
\hline 68 & Caragana brevispina Benth. & S & SPS 4535 & June-Aug. & $2550-2800$ & CT & Occasional, under Deodar forests \\
\hline 69 & Hedysarum microcalyx Baker & $\mathrm{H}$ & SPS 3906 & June-Aug. & 2700-3000 & CT & Rare, along stream sides, wet slopes \\
\hline 70 & Indigofera heterantha Brandis & SS & SPS 3898 & May-Sep. & $1900-2500$ & $\mathrm{~N}$ & Common, in open slopes \\
\hline 71 & Indigofera hebepetala Baker & SS & SPS 3810 & May-Aug. & $2400-2650$ & $\mathrm{~N}$ & Occasional, in shady places \\
\hline 72 & Lathyrus emodi (Fritsch) Ali & $\mathrm{H}$ & SPS 4034 & April-May & $2400-2650$ & CT & $\begin{array}{l}\text { Occasional, in foothills and with } \\
\text { waysides }\end{array}$ \\
\hline 73 & $\begin{array}{l}\text { Piptanthus nepalensis (Hook.) D. } \\
\text { Don }\end{array}$ & $\mathrm{s}$ & SPS 4086 & Mar.-May & $2700-2950$ & C & $\begin{array}{l}\text { Occasional, under mixed conifer } \\
\text { forests }\end{array}$ \\
\hline 74 & Sophora mollis (Royle) Baker & S & SPS 4313 & May-July & $1900-2150$ & C & $\begin{array}{l}\text { Rare, in foothills, hill slopes, } \\
\text { associated with Ban oak forests }\end{array}$ \\
\hline 75 & Trifolium dubium Sibth. & $\mathrm{H}$ & SPS 3711 & April-July & $2400-3000$ & $\mathrm{~N}$ & Common, in moist, shady places \\
\hline 76 & Trifolium pratense $\mathrm{L}$. & $\mathrm{H}$ & $\begin{array}{l}\text { SPS 3672, } \\
3678\end{array}$ & May-Sep. & $2300-3400$ & $\mathrm{~N}$ & Common, in moist, shady places \\
\hline 77 & Trigonella emodi Benth. & $\mathrm{H}$ & SPS 4567 & June-Sep. & $3150-3370$ & CT & Common, along pathways \\
\hline 78 & Trigonella gracilis Benth. & $\mathrm{H}$ & SPS 4527 & July-Sep. & $3200-3450$ & $\mathrm{~N}, \mathrm{C}$ & Common, along pathways \\
\hline \multirow[t]{2}{*}{79} & Vicia tenera Benth. & $\mathrm{Cl}$ & SPS 3921 & April-June & $2200-2450$ & $\mathrm{~N}$ & Occasional, in the forest outskirts \\
\hline & Caesalpinioideae & & & & & & \\
\hline 80 & $\begin{array}{l}\text { Chamaecrista nomame (Sieber) H. } \\
\text { Ohashi }\end{array}$ & $\mathrm{H}$ & SPS 3950 & July-Oct. & $1900-2200$ & N & Common, in exposed hilly slopes \\
\hline \multicolumn{8}{|c|}{ Rosaceae } \\
\hline 81 & Agrimonia pilosa Ledeb. & $\mathrm{H}$ & SPS 4163 & July-Sep. & $1900-2200$ & $\mathrm{~N}$ & $\begin{array}{l}\text { Common, under shrubberies in } \\
\text { conifers and Oak forests. }\end{array}$ \\
\hline 82 & $\begin{array}{l}\text { Cerasus cerasoides (Buch.-Ham. ex } \\
\text { D.Don) S.Y. Sokolov }\end{array}$ & ST & SPS 4423 & April-May & $1900-2550$ & C, CT & $\begin{array}{l}\text { Occasional, in open hilly sides, } \\
\text { associated with Lyonia and Ban } \\
\text { oaks. }\end{array}$ \\
\hline 83 & Cotoneaster bacillaris Wall. ex Lindl. & s & SPS 3565 & May-Oct. & $2450-2900$ & $\mathrm{~N}$ & Occasional, along with waysides \\
\hline 84 & Cotoneaster roseus Edgew. & S & SPS 3616 & May-Aug. & $2950-3400$ & C & $\begin{array}{l}\text { Occasional, associated with Rosa } \\
\text { macrophylla, Viburnum spp. }\end{array}$ \\
\hline 85 & $\begin{array}{l}\text { Filipendula vestita (Wall. ex G.Don) } \\
\text { Maxim. }\end{array}$ & $\mathrm{H}$ & SPS 4357 & July-Sep. & $3400-3650$ & $\mathrm{~N}$ & $\begin{array}{l}\text { Occasional, in subalpine shrubbries } \\
\text { open slopes below Chur peak }\end{array}$ \\
\hline 86 & $\begin{array}{l}\text { Potentilla fruticosa L. var. arbuscula } \\
\text { (D. Don) Maxim. }\end{array}$ & $\mathrm{H}$ & SPS 3698 & June-Sep. & $2950-3500$ & $\mathrm{~N}$ & $\begin{array}{l}\text { Frequent, in open alpine slopes } \\
\text { below Chur peak }\end{array}$ \\
\hline 87 & Potentilla supina $\mathrm{L}$. & $\mathrm{H}$ & $\begin{array}{l}\text { SPS 3507, } \\
3641\end{array}$ & July-Aug. & $3000-3400$ & C & $\begin{array}{l}\text { Frequent, in open alpine slopes } \\
\text { below Chur peak }\end{array}$ \\
\hline 88 & $\begin{array}{l}\text { Rosa macrophylla Lindl. var. minor } \\
\text { Lindl. ex Wall. }\end{array}$ & CS & SPS 3609 & May-Oct. & $2650-3150$ & $\mathrm{~N}$ & $\begin{array}{l}\text { Rare, with shrubberies under } \\
\text { Kharshu oak and conifer forests }\end{array}$ \\
\hline
\end{tabular}




\begin{tabular}{|c|c|c|c|c|c|c|c|}
\hline & Botanical Name & Habit & $\begin{array}{l}\text { HFRI } \\
\text { collection } \\
\text { no. }\end{array}$ & $\begin{array}{l}\text { Flowering \& } \\
\text { fruiting }\end{array}$ & $\begin{array}{l}\text { Altitudinal } \\
\text { range }(\mathrm{m})\end{array}$ & Forest area & Remarks \\
\hline 89 & Rubus paniculatus Sm. & CS & SPS 4454 & April-July & $2300-2550$ & c & $\begin{array}{l}\text { Occasional, in forest outskirts, Ban } \\
\text { oak forests }\end{array}$ \\
\hline 90 & Sorbaria tomentosa (Lindl.) Rehder & $S$ & SPS 3867 & May-July & $2200-2550$ & $\mathrm{~N}$ & Frequent, in foothills, moist slopes \\
\hline \multicolumn{8}{|c|}{ Saxifragaceae } \\
\hline 91 & $\begin{array}{l}\text { Astilbe rivularis Buch.-Ham. ex } \\
\text { D.Don }\end{array}$ & $\mathrm{H}$ & SPS 4534 & June-Aug. & $3050-3300$ & $\mathrm{~N}, \mathrm{C}, \mathrm{CT}$ & $\begin{array}{l}\text { Frequent, as shrubbries in moist, } \\
\text { shady places }\end{array}$ \\
\hline 92 & Saxifraga brachypoda D.Don & $\mathrm{H}$ & SPS 4000 & June-Sep. & $3400-3600$ & $\mathrm{~N}$ & $\begin{array}{l}\text { Rare, on wet rocks and in alpine } \\
\text { meadows }\end{array}$ \\
\hline 93 & Saxifraga brunonis Wall. ex Ser. & $\mathrm{H}$ & \begin{tabular}{|l} 
SPS 4040, \\
3806 \\
\end{tabular} & July-Sep. & $2900-3200$ & $\mathrm{~N}, \mathrm{CT}$ & Occasional, in moist, shady places \\
\hline 94 & $\begin{array}{l}\text { Saxifraga moorcroftiana (Ser.) Wall. } \\
\text { ex Sternb. }\end{array}$ & $\mathrm{H}$ & SPS 3594 & July-Sep. & $3200-3600$ & $\mathrm{~N}, \mathrm{C}$ & $\begin{array}{l}\text { Rare, in alpine meadows below } \\
\text { Chur peak }\end{array}$ \\
\hline 95 & Saxifraga mucronulata Royle & $\mathrm{H}$ & SPS 3540 & July-Sep. & $3200-3600$ & $N$ & Rare, in alpine meadows \\
\hline 96 & Saxifraga pallida Wall. ex Ser. & $\mathrm{H}$ & SPS 3739 & June-Aug. & $3000-3200$ & $\mathrm{~N}$ & Occasional, on wet rocks \\
\hline 97 & Saxifraga sibirica L. & $\mathrm{H}$ & SPS 4142 & June-Aug. & $2700-3400$ & $\mathrm{~N}$ & Occasional, on dripping rocky slopes \\
\hline \multicolumn{8}{|c|}{ Philadelphaceae } \\
\hline 98 & Deutzia corymbosa R.Br. ex G.Don & $s$ & SPS 4420 & May-Aug. & $2450-2900$ & C & Rare, in exposed, hilly slopes \\
\hline \multicolumn{8}{|c|}{ Grossulariaceae } \\
\hline 99 & Ribes alpestre Wall. ex Decne. & $S$ & SPS 3603 & Aug.-Sep. & $2950-3200$ & $N$ & $\begin{array}{l}\text { Rare, spreading over on exposed } \\
\text { rocks below Chur peak }\end{array}$ \\
\hline 100 & Ribes glaciale Wall. & $S$ & \begin{tabular}{|l} 
SPS 3568, \\
3717,3853, \\
3863
\end{tabular} & June-July & $2950-3600$ & $\mathrm{~N}, \mathrm{C}$ & $\begin{array}{l}\text { Rare, spreading over on exposed } \\
\text { rocks below Chur peak }\end{array}$ \\
\hline 101 & Ribes orientale Desf. & $S$ & SPS 3572 & July-Sep. & $3200-3450$ & CT & $\begin{array}{l}\text { Rare, on spreading over exposed } \\
\text { rocks below Chur peak }\end{array}$ \\
\hline \multicolumn{8}{|c|}{ Crassulaceae } \\
\hline 102 & $\begin{array}{l}\text { Hylotelephium ewersii (Ledeb.) H. } \\
\text { Ohba }\end{array}$ & $\mathrm{H}$ & $\begin{array}{l}\text { SPS 4003, } \\
4183\end{array}$ & July-Sep. & $2900-3600$ & $N$ & $\begin{array}{l}\text { Frequent, on wet rocks in alpine } \\
\text { and subalpine meadows }\end{array}$ \\
\hline 103 & $\begin{array}{l}\text { Rhodiola heterodonta (Hook. f. \& } \\
\text { Thomson) Boriss. }\end{array}$ & $\mathrm{H}$ & SPS 3852 & June-Aug. & $2900-3600$ & $N$ & Rare, in alpine meadows \\
\hline 104 & Rhodiola himalensis (D. Don) S.H. Fu & $\mathrm{H}$ & SPS 3619 & June-Sep. & $3200-3640$ & $\mathrm{~N}$ & Rare, in alpine meadows \\
\hline 105 & $\begin{array}{l}\text { Rhodiola tibetica (Hook. f. \& } \\
\text { Thomson) S.H. Fu }\end{array}$ & $\mathrm{H}$ & SPS 3947 & July-Sep. & $3150-3400$ & CT & $\begin{array}{l}\text { Rare, in alpine meadows below } \\
\text { Chur peak }\end{array}$ \\
\hline 106 & Rhodiola wallichiana (Hook.) S.H. Fu & $\mathrm{H}$ & SPS 3988 & July-Sep. & $3000-3550$ & $\mathrm{~N}, \mathrm{CT}$ & $\begin{array}{l}\text { Rare, in alpine meadows below } \\
\text { Chur peak }\end{array}$ \\
\hline 107 & $\begin{array}{l}\text { Rosularia adenotricha (Wall. ex } \\
\text { Edgew.) C.A. Jansson }\end{array}$ & $\mathrm{H}$ & SPS 3892 & May-June & $2700-3450$ & $\mathrm{~N}$ & Occasional, on wet rocks \\
\hline \multicolumn{8}{|c|}{ Lythraceae } \\
\hline 108 & $\begin{array}{l}\text { Rotala rotundifolia (Buch.-Ham. ex } \\
\text { Roxb.) Koehne }\end{array}$ & $\mathrm{H}$ & SPS 4501 & Mar.-June & $1900-2300$ & $N$ & Occasional, in damp places \\
\hline \multicolumn{8}{|c|}{ Onagraceae } \\
\hline 109 & $\begin{array}{l}\text { Circaea alpina L. subsp. imaicola } \\
\text { (Asch. \& Magnus) Kitam. }\end{array}$ & $\mathrm{H}$ & \begin{tabular}{|l} 
SPS 4074, \\
4343 \\
\end{tabular} & July-Sep. & $2900-3400$ & $\mathrm{~N}, \mathrm{C}$ & Occasional, along water courses \\
\hline 110 & Epilobium brevifolium D. Don & $\mathrm{H}$ & SPS 3769 & June-Aug. & $2450-3500$ & $\mathrm{~N}$ & $\begin{array}{l}\text { Occasional, in degraded forest } \\
\text { slopes }\end{array}$ \\
\hline 111 & Epilobium cylindricum D. Don & $\mathrm{H}$ & SPS 4448 & Aug.-Oct. & $2400-3200$ & $N$ & $\begin{array}{l}\text { Frequent, in exposed slopes, grazed } \\
\text { areas }\end{array}$ \\
\hline 112 & Epilobium latifolium L. & $\mathrm{H}$ & SPS 4368 & June-Sep. & $2150-3200$ & $N$ & $\begin{array}{l}\text { Common, along water courses in } \\
\text { alpine meadows }\end{array}$ \\
\hline 113 & Epilobium laxum Royle & $\mathrm{H}$ & \begin{tabular}{|l} 
SPS 3721, \\
3726,3986 \\
\end{tabular} & July-Oct. & $3150-3600$ & $N$ & $\begin{array}{l}\text { Occasional, in shady places, under } \\
\text { shrubberies }\end{array}$ \\
\hline 114 & Epilobium royleanum Hausskn. & $\mathrm{H}$ & SPS 4317 & Aug.-Nov. & $2400-3600$ & $\mathrm{~N}$ & Frequent, in damp places \\
\hline \multicolumn{8}{|c|}{ Cucurbitaceae } \\
\hline 115 & Solena amplexicaulis (Lam.) Gandhi & $\mathrm{Cl}$ & SPS 4444 & May-Aug. & $1900-2100$ & c & $\begin{array}{l}\text { Frequent, on thickets, forest } \\
\text { outskirts }\end{array}$ \\
\hline \multicolumn{8}{|c|}{ Apiaceae } \\
\hline 116 & $\begin{array}{l}\text { Acronema hookeri (C.B. Clarke) H. } \\
\text { Wolff }\end{array}$ & $\mathrm{H}$ & SPS 4161 & June-July & $3400-3550$ & $\mathrm{~N}$ & Rare, in alpine meadows \\
\hline 117 & Angelica glauca Edgew. & $\mathrm{H}$ & SPS 3843 & July-Aug. & $2900-3400$ & C & Rare, in subalpine slopes \\
\hline 118 & Bupleurum aitchisonii $\mathrm{H}$. Wolff & $\mathrm{H}$ & $\begin{array}{l}\text { SPS 3991, } \\
4072\end{array}$ & July-Sep. & $3000-3400$ & c & $\begin{array}{l}\text { Rare, among rock boulders, below } \\
\text { Chur peak }\end{array}$ \\
\hline
\end{tabular}




\begin{tabular}{|c|c|c|c|c|c|c|c|}
\hline & Botanical Name & Habit & $\begin{array}{l}\text { HFRI } \\
\text { collection } \\
\text { no. }\end{array}$ & $\begin{array}{l}\text { Flowering \& } \\
\text { fruiting }\end{array}$ & $\begin{array}{l}\text { Altitudinal } \\
\text { range }(\mathrm{m})\end{array}$ & Forest area & Remarks \\
\hline 119 & Bupleurum falcatum L. & $\mathrm{H}$ & SPS 4299 & May-Sep. & $2150-3200$ & $N$ & $\begin{array}{l}\text { Common, in shady places under } \\
\text { Deodar forests }\end{array}$ \\
\hline 120 & Bupleurum lanceolatum Wall. ex DC. & $\mathrm{H}$ & SPS 3516 & July-Oct. & $2150-2900$ & $\mathrm{~N}$ & $\begin{array}{l}\text { Frequent, in shady places under } \\
\text { Deodar forests }\end{array}$ \\
\hline 121 & Bupleurum maddenii C.B. Clarke & $\mathrm{H}$ & SPS 3505 & May-July & $3150-3400$ & $\mathrm{~N}, \mathrm{C}, \mathrm{CT}$ & $\begin{array}{l}\text { Rare, between boulders, below } \\
\text { Chur peak }\end{array}$ \\
\hline 122 & $\begin{array}{l}\text { Chaerophyllum reflexum var. } \\
\text { acuminatum (Lindl.) Hedge \& } \\
\text { Lamond }\end{array}$ & $\mathrm{H}$ & SPS 4364 & June-Sep. & $2700-3370$ & $N$ & Frequent, in moist, exposed slopes \\
\hline 123 & Chaerophyllum villosum Wall. ex DC. & $\mathrm{H}$ & SPS 3912 & June-Aug. & $2450-3150$ & $\mathrm{~N}$ & Common, in moist, exposed slopes \\
\hline 124 & $\begin{array}{l}\text { Pleurospermum brunonis Benth. ex } \\
\text { C.B. Clarke }\end{array}$ & $\mathrm{H}$ & $\begin{array}{l}\text { SPS 3967, } \\
4024,4066 \\
\end{array}$ & July-Sep. & $3150-3600$ & N & $\begin{array}{l}\text { Rare, in alpine meadows and in } \\
\text { open rocky crevices }\end{array}$ \\
\hline 125 & $\begin{array}{l}\text { Pleurospermum candollei Benth. ex } \\
\text { C.B. Clarke }\end{array}$ & $\mathrm{H}$ & SPS 3512 & July-Sep. & $3400-3640$ & $N$ & $\begin{array}{l}\text { Rare, in alpine slopes, between } \\
\text { boulders, Nohra beat }\end{array}$ \\
\hline 126 & $\begin{array}{l}\text { Pleurospermum stellatum (D.Don) } \\
\text { Benth. ex C.B. Clarke }\end{array}$ & $\mathrm{H}$ & SPS 3924 & July-Sep. & $3400-3640$ & $N$ & Rare, in alpine meadows \\
\hline 127 & Selinum vaginatum C.B. Clarke & $\mathrm{H}$ & $\begin{array}{l}\text { SPS 3976, } \\
4038\end{array}$ & July-Sep. & $2900-3300$ & $\mathrm{~N}, \mathrm{C}$ & Rare, in subalpine scrub and slopes \\
\hline 128 & Seseli libanotis (L.) Koch & $\mathrm{H}$ & SPS 3859 & July-Sep. & $2900-3200$ & $N$ & $\begin{array}{l}\text { Occasional, among boulders below } \\
\text { Chur peak }\end{array}$ \\
\hline \multicolumn{8}{|c|}{ Cornaceae } \\
\hline 129 & Cornus macrophylla Wall. & $T$ & $\begin{array}{l}\text { SPS 3547, } \\
3555\end{array}$ & July-Aug. & $2400-2700$ & N. CT & $\begin{array}{l}\text { Occasional, along water channels } \\
\text { at foothills }\end{array}$ \\
\hline \multicolumn{8}{|c|}{ Alangiaceae } \\
\hline 130 & Alangium chinense (Lour.) Harms & ST & SPS 4185 & Mar.-July & $2150-2400$ & C & Occasional, in outskirts of forests \\
\hline \multicolumn{8}{|c|}{ Caprifoliaceae } \\
\hline 131 & Leycesteria formosa Wall. & $\mathrm{s}$ & \begin{tabular}{|l} 
SPS 4087, \\
4319 \\
\end{tabular} & May-July & $2650-2900$ & $\mathrm{~N}, \mathrm{CT}$ & $\begin{array}{l}\text { Frequent, shrubbries under Oak } \\
\text { forests }\end{array}$ \\
\hline 132 & Lonicera angustifolia Wall. ex DC. & SS & SPS 4565 & May-Oct. & $2500-3100$ & C & Occasional, in exposed forests \\
\hline 133 & $\begin{array}{l}\text { Lonicera asperifolia Hook. f. \& } \\
\text { Thomson }\end{array}$ & $\mathrm{s}$ & SPS 3562 & May-July & $2900-3300$ & $N$ & $\begin{array}{l}\text { Occasional, spreading over rocks at } \\
\text { subalpines meadows }\end{array}$ \\
\hline 134 & $\begin{array}{l}\text { Lonicera hispida Pall. ex Roem. \& } \\
\text { Schult. var. bracteata (Royle) Rehder } \\
\text { ex Airy Shaw }\end{array}$ & $\mathrm{s}$ & SPS 3530 & July-Sep. & $2900-3200$ & $\mathrm{~N}$ & $\begin{array}{l}\text { Occasional, spreading over rocks at } \\
\text { subalpines meadows }\end{array}$ \\
\hline 135 & Lonicera hypoleuca Decne. & $\mathrm{s}$ & SPS 3710 & June-Oct. & $2550-2900$ & $\mathrm{~N}, \mathrm{C}$ & $\begin{array}{l}\text { Frequent, hanging over on stony } \\
\text { slopes }\end{array}$ \\
\hline 136 & Lonicera obovata Royle & $\mathrm{s}$ & $\begin{array}{l}\text { SPS 3613, } \\
3741,4141 \\
\end{array}$ & Mar.-July & $2950-3600$ & $\mathrm{~N}$ & $\begin{array}{l}\text { Rare, on spreading over rocks in } \\
\text { alpine meadows }\end{array}$ \\
\hline 136 & Lonicera orientalis Lam. & $s$ & SPS 4116 & June-Aug. & 2700-3300 & $\mathrm{N}$ & Occasional, in exposed stony slopes \\
\hline 137 & Lonicera quinquelocularis Hardw. & $\mathrm{s}$ & SPS 3593 & June-Sep. & $2400-2900$ & $\mathrm{~N}, \mathrm{CT}$ & $\begin{array}{l}\text { Frquent, in Oaks \& conifer forest } \\
\text { slopes }\end{array}$ \\
\hline 139 & Lonicera webbiana Wall. ex DC. & $\mathrm{s}$ & SPS 4559 & July-Aug. & $2650-3200$ & $\mathrm{~N}, \mathrm{C}$ & Rare, in forest outskirts \\
\hline 140 & $\begin{array}{l}\text { Viburnum mullaha Buch.-Ham. ex } \\
\text { D.Don }\end{array}$ & $s$ & $\begin{array}{l}\text { SPS } 3543, \\
3586,3873 \\
3899\end{array}$ & Sep.-Oct. & $2100-2400$ & $\mathrm{~N}, \mathrm{CT}$ & Frequent, along water-channels \\
\hline \multicolumn{8}{|c|}{ Rubiaceae } \\
\hline 141 & Argostemma verticillatum $\mathrm{L}$. & $\mathrm{H}$ & SPS 4361 & Aug.-Oct. & $2150-2500$ & C & Common, in moist stony slopes \\
\hline 142 & Neanotis hirsuta (L.f.) W.H. Lewis & $\mathrm{H}$ & SPS 4416 & July-Oct. & $1900-2100$ & $\mathrm{~N}, \mathrm{C}$ & $\begin{array}{l}\text { Common, foothills and in open } \\
\text { grasslands }\end{array}$ \\
\hline \multicolumn{8}{|c|}{ Valerianaceae } \\
\hline 143 & Valeriana hardwickii Wall. & $\mathrm{H}$ & $\begin{array}{l}\text { SPS 4098, } \\
4362,4089, \\
4341\end{array}$ & May-Sep. & $2400-2700$ & $\mathrm{~N}$ & $\begin{array}{l}\text { Rare, in shady places under conifer } \\
\text { forests }\end{array}$ \\
\hline \multicolumn{8}{|c|}{ Asteraceae } \\
\hline 144 & Ainsliaea aptera DC. & $\mathrm{H}$ & SPS 3914 & Mar.-Sep. & $2650-3100$ & $N$ & $\begin{array}{l}\text { Rare, in humus rich soils, under } \\
\text { shrubberies }\end{array}$ \\
\hline 145 & Ainsliaea latifolia (D.Don) Sch. Bip. & $\mathrm{H}$ & SPS 4366 & Mar.-May & $2000-3100$ & $N$ & $\begin{array}{l}\text { Frequent, in humus rich soils, under } \\
\text { Deodar \& Kharshu oak forests }\end{array}$ \\
\hline 146 & $\begin{array}{l}\text { Anaphalis nepalensis (Spreng.) } \\
\text { Hand.-Mazz. }\end{array}$ & $\mathrm{H}$ & SPS 3990 & June-Sep. & $3300-3600$ & $N$ & Frequent, in alpine meadows \\
\hline 147 & $\begin{array}{l}\text { Anaphalis triplinervis (Sims) Sims ex } \\
\text { C.B. Clarke }\end{array}$ & $\mathrm{H}$ & SPS 4294 & July-Sep. & $2900-3600$ & $N$ & Common, in alpine meadows \\
\hline
\end{tabular}




\begin{tabular}{|c|c|c|c|c|c|c|c|}
\hline & Botanical Name & Habit & \begin{tabular}{|l} 
HFRI \\
collection \\
no. \\
\end{tabular} & $\begin{array}{l}\text { Flowering \& } \\
\text { fruiting }\end{array}$ & $\begin{array}{l}\text { Altitudinal } \\
\text { range }(\mathrm{m})\end{array}$ & Forest area & Remarks \\
\hline 148 & Artemisia indica Willd. & $\mathrm{H}$ & SPS 3994 & July-Oct. & $1900-2800$ & c & $\begin{array}{l}\text { Frequent, in exposed hilly slopes, } \\
\text { grazed areas }\end{array}$ \\
\hline 149 & $\begin{array}{l}\text { Aster molliusculus (Lindl. ex DC.) } \\
\text { C.B.Clarke }\end{array}$ & $\mathrm{H}$ & SPS 3668 & May-Sep. & $2200-2900$ & $N$ & Common, in exposed waste places \\
\hline 150 & Bidens tripartita $\mathrm{L}$. & $\mathrm{H}$ & SPS 4293 & Aug.-Oct. & $1900-2400$ & $\mathrm{~N}$ & $\begin{array}{l}\text { Common, in waste places and in } \\
\text { exposed slopes }\end{array}$ \\
\hline 151 & Carpesium cernuum L. & $\mathrm{H}$ & SPS 4187 & July-Sep. & $1900-2150$ & c & $\begin{array}{l}\text { Frequent, along roadsides and } \\
\text { pathways }\end{array}$ \\
\hline 152 & $\begin{array}{l}\text { Cephalorrhynchus macrorhizus } \\
\text { (Royle) Tuisl }\end{array}$ & $\mathrm{H}$ & $\begin{array}{l}\text { SPS 3962, } \\
4047 \\
\end{array}$ & June-Sep. & $2900-3600$ & $N$ & Rare, in alpine slopes, rocky crevices \\
\hline 153 & Erigeron alpinus $\mathrm{L}$. & $\mathrm{H}$ & SPS 4252 & May-Sep. & $2900-3600$ & $\mathrm{~N}, \mathrm{C}$ & $\begin{array}{l}\text { Common, in exposed slopes and in } \\
\text { subalpine meadows }\end{array}$ \\
\hline 154 & $\begin{array}{l}\text { Erigeron multiradiatus (Lindl. ex DC.) } \\
\text { C.B. Clarke }\end{array}$ & $\mathrm{H}$ & SPS 4320 & April-Sep. & $2200-2900$ & $\mathrm{~N}, \mathrm{C}, \mathrm{CT}$ & $\begin{array}{l}\text { Common in exposed slopes and in } \\
\text { shady places }\end{array}$ \\
\hline 155 & Gerbera gossypina (Royle) Beauv. & $\mathrm{H}$ & SPS 4412 & May-Sep. & $2000-2900$ & $\mathrm{~N}, \mathrm{C}, \mathrm{CT}$ & Common, in moist, open hilly slopes \\
\hline 156 & $\begin{array}{l}\text { Lactuca brunoniana (Wall. ex DC.) } \\
\text { C.B. Clarke }\end{array}$ & $\mathrm{H}$ & SPS 4084 & June-Oct & $2450-3100$ & $\mathrm{~N}, \mathrm{C}, \mathrm{CT}$ & $\begin{array}{l}\text { Frequent, in humus rich soils, shady } \\
\text { places }\end{array}$ \\
\hline 157 & Ligularia amplexicaulis DC. & $\mathrm{H}$ & $\begin{array}{l}\text { SPS 4064, } \\
4291 \\
\end{array}$ & July-Oct & $2650-3100$ & $\mathrm{~N}, \mathrm{C}, \mathrm{CT}$ & $\begin{array}{l}\text { Common, under Abies spectabilis } \\
\text { forests }\end{array}$ \\
\hline 158 & Saussurea auriculata (DC.) Sch. Bip. & $\mathrm{H}$ & SPS 3519 & July-Sep. & $2950-3400$ & $\mathrm{~N}$, & Frequent, in alpine slopes \\
\hline 159 & Saussurea piptathera Edgew. & $\mathrm{H}$ & SPS 4045 & July-Sep. & $3200-3600$ & $\mathrm{~N}$ & $\begin{array}{l}\text { Common, in alpine and subalpine } \\
\text { meadows below Chur peak }\end{array}$ \\
\hline 160 & Saussurea taraxacifolia Wall. ex DC. & $\mathrm{H}$ & $\begin{array}{l}\text { SPS 3520, } \\
4063\end{array}$ & July-Sep. & $3150-3640$ & $\mathrm{~N}, \mathrm{C}$ & $\begin{array}{l}\text { Common, in alpine meadows below } \\
\text { Chur peak }\end{array}$ \\
\hline 161 & Senecio kunthianus Wall. ex DC. & $\mathrm{H}$ & SPS 4044 & July-Sep. & $3150-3640$ & $\mathrm{~N}, \mathrm{C}, \mathrm{CT}$ & $\begin{array}{l}\text { Common, in alpine meadows below } \\
\text { Chur peak }\end{array}$ \\
\hline 162 & Senecio rufinervis DC. & $\mathrm{H}$ & SPS 3508 & Aug.-Oct. & $2400-3150$ & $\mathrm{~N}, \mathrm{C}, \mathrm{CT}$ & $\begin{array}{l}\text { Frequent, in Conifer-Kharshu oak } \\
\text { forests }\end{array}$ \\
\hline 163 & Tanacetum longifolium Wall. ex DC. & $\mathrm{H}$ & SPS 4458 & July-Oct. & $2900-3600$ & $\mathrm{~N}, \mathrm{C}, \mathrm{CT}$ & Common, in alpine meadows \\
\hline \multicolumn{8}{|c|}{ Campanulaceae } \\
\hline 164 & $\begin{array}{l}\text { Campanula argyrotricha Wall. ex } \\
\text { A. DC. }\end{array}$ & $\mathrm{H}$ & SPS 3876 & May-Sep. & $2950-3400$ & N & $\begin{array}{l}\text { Rare, on rocky crevices below Chur } \\
\text { peak }\end{array}$ \\
\hline 165 & Campanula latifolia $\mathrm{L}$. & $\mathrm{H}$ & SPS 3808 & June-Sep. & $2400-2900$ & $\mathrm{~N}, \mathrm{C}, \mathrm{CT}$ & $\begin{array}{l}\text { Frequent, under shrubberies in } \\
\text { conifer forests }\end{array}$ \\
\hline \multicolumn{8}{|c|}{ Ericaceae } \\
\hline 166 & Cassiope fastigiata (Wall.) D. Don & $\mathrm{H}$ & \begin{tabular}{|l} 
SPS 3701, \\
3992 \\
\end{tabular} & June-Sep. & $3000-3600$ & $N$ & $\begin{array}{l}\text { Frequent, on wet rocks in alpine } \\
\text { scrub }\end{array}$ \\
\hline 167 & Gaultheria trichophylla Royle & $\mathrm{H}$ & SPS 3954 & June-Aug. & $3150-3600$ & $N$ & Common, in alpine slopes \\
\hline 168 & Lyonia ovalifolia (Wall.) Drude & $T$ & SPS 3998 & May-July & $1900-2800$ & $\mathrm{~N}, \mathrm{C}, \mathrm{CT}$ & $\begin{array}{l}\text { Common, in association with Ban } \\
\text { oaks \& Rhododendron arboreum }\end{array}$ \\
\hline 169 & Rhododendron anthopogon D. Don & $S$ & $\begin{array}{l}\text { SPS 3558, } \\
3980,4028 \\
\end{array}$ & June-Sep. & $3150-3500$ & $N$ & Rare, in subalpine scrub \\
\hline 170 & $\begin{array}{l}\text { Rhododendron lepidotum Wall. ex } \\
\text { G.Don }\end{array}$ & $s$ & $\begin{array}{l}\text { SPS 3622, } \\
3706\end{array}$ & June-Oct. & $3150-3600$ & $N$ & $\begin{array}{l}\text { Rare, in subalpine scrub, rocky } \\
\text { crevices }\end{array}$ \\
\hline \multicolumn{8}{|c|}{ Primulaceae } \\
\hline 171 & $\begin{array}{l}\text { Androsace sempervivoides Jacq. } \\
\text { ex Duby }\end{array}$ & $\mathrm{H}$ & SPS 4288 & April-July & $2400-2700$ & $N$ & $\begin{array}{l}\text { Occasional, in subalpine, alpine } \\
\text { slopes }\end{array}$ \\
\hline 172 & Cortusa brotheri Pax ex Lipsky & $\mathrm{H}$ & SPS 3513 & July-Aug. & $3000-3300$ & $N$ & $\begin{array}{l}\text { Rare, in shady places, in ledges of } \\
\text { rocks below Chur peak }\end{array}$ \\
\hline 173 & Lysimachia ferruginea Edgew. & $\mathrm{H}$ & \begin{tabular}{|l} 
SPS 3628, \\
3918,3936 \\
\end{tabular} & June-Aug. & $1900-2450$ & $\mathrm{~N}, \mathrm{C}$ & $\begin{array}{l}\text { Frequent, in dripping slopes, along } \\
\text { rivulets }\end{array}$ \\
\hline 174 & Primula involucrata Wall. ex Duby & $\mathrm{H}$ & SPS 3522 & July-Sep. & $3200-3600$ & $N$ & $\begin{array}{l}\text { Occasional, wet places, in alpine } \\
\text { meadows }\end{array}$ \\
\hline 175 & Primula petiolaris Wall. & $\mathrm{H}$ & $\begin{array}{l}\text { SPS 3502, } \\
3932\end{array}$ & April-Sep. & $2900-3400$ & $\mathrm{~N}, \mathrm{C}$ & $\begin{array}{l}\text { Occasional, in wet places, alpine } \\
\text { meadows }\end{array}$ \\
\hline 176 & Primula reidii Duthie & $\mathrm{H}$ & $\begin{array}{l}\text { SPS 3734, } \\
4048\end{array}$ & June-Aug. & $3000-3400$ & $\mathrm{~N}, \mathrm{CT}$ & $\begin{array}{l}\text { Rare, shady places in subalpine } \\
\text { scrub }\end{array}$ \\
\hline \multicolumn{8}{|c|}{ Myrsinaceae } \\
\hline 177 & Myrsine semiserrata Wall. & $\mathrm{H}$ & $\begin{array}{l}\text { SPS 3734, } \\
4048\end{array}$ & Sep.-Nov. & $2000-2300$ & C, CT & $\begin{array}{l}\text { Occasional, in foothills and in Ban } \\
\text { oak forests }\end{array}$ \\
\hline \multicolumn{8}{|c|}{ Oleaceae } \\
\hline 178 & Chionanthus ramiflorus Roxb. & ST & SPS 3689 & April-Aug. & $2600-2800$ & c & Rare, along rivulets \\
\hline
\end{tabular}




\begin{tabular}{|c|c|c|c|c|c|c|c|}
\hline & Botanical Name & Habit & $\begin{array}{l}\text { HFRI } \\
\text { collection } \\
\text { no. }\end{array}$ & $\begin{array}{l}\text { Flowering \& } \\
\text { fruiting }\end{array}$ & $\begin{array}{l}\text { Altitudinal } \\
\text { range }(\mathrm{m})\end{array}$ & Forest area & Remarks \\
\hline 179 & Syringa emodi Wall. ex Royle & ST & SPS 4337 & May-July & $2900-3400$ & $N$ & $\begin{array}{l}\text { Common, associated with Betula- } \\
\text { Rhododendron forests }\end{array}$ \\
\hline \multicolumn{8}{|c|}{ Asclepiadaceae } \\
\hline 180 & Pergularia roylei (Wight) D. Dietr. & CS & SPS 3913 & May-Sep. & $2000-2200$ & CT & $\begin{array}{l}\text { Occasional, at foothills on thickets, } \\
\text { Deodar and Ban oak forests }\end{array}$ \\
\hline \multicolumn{8}{|c|}{ Gentianaceae } \\
\hline 181 & Comastoma tenellum (Rottb.) Toyok. & $\mathrm{H}$ & SPS 4523 & July-Sep. & 2950-3300 & $N$ & Common in alpine meadows \\
\hline 182 & Gentianopsis paludosa (Hook.f.) Ma & $\mathrm{H}$ & SPS 3523 & July-Sep. & $2900-3400$ & $N$ & Frequent, in open alpine slopes \\
\hline 183 & Halenia elliptica D.Don & $\mathrm{H}$ & $\begin{array}{l}\text { SPS 3794, } \\
4051\end{array}$ & Aug.-Sep. & $2400-3300$ & $\mathrm{~N}, \mathrm{CT}$ & $\begin{array}{l}\text { Frequent, in alpine and subalpine } \\
\text { slopes }\end{array}$ \\
\hline 184 & $\begin{array}{l}\text { Jaeschkea canaliculata (Royle ex } \\
\text { G.Don) Knobl. }\end{array}$ & $\mathrm{H}$ & SPS 4287 & July-Sep. & $2950-3200$ & $N$ & Frequent, in subalpine slopes \\
\hline 185 & Swertia alternifolia Royle & $\mathrm{H}$ & SPS 3750 & Aug.-Sep. & $3150-3370$ & $N$ & $\begin{array}{l}\text { Rare, in subalpines under } \\
\text { Rhododendron campanulatum } \\
\text { forests }\end{array}$ \\
\hline 186 & $\begin{array}{l}\text { Swertia ciliata (D.Don ex G. Don) } \\
\text { B.L. Burtt }\end{array}$ & $\mathrm{H}$ & $\begin{array}{l}\text { SPS 3549, } \\
3550 \\
\end{array}$ & Aug.-Nov. & $2150-3300$ & $\mathrm{~N}, \mathrm{CT}$ & $\begin{array}{l}\text { Common, in grassy slopes and in } \\
\text { exposed places }\end{array}$ \\
\hline 187 & Swertia cuneata Wall. ex D.Don & $\mathrm{H}$ & \begin{tabular}{|l|} 
SPS 3748, \\
4157 \\
\end{tabular} & Aug.-Sep. & $3150-3600$ & $N, C$ & $\begin{array}{l}\text { Frequent, in alpine meadows, below } \\
\text { Chur peak }\end{array}$ \\
\hline 188 & Swertia speciosa D.Don & $\mathrm{H}$ & SPS 3535 & Aug.-Sep. & $2900-3600$ & $N$ & $\begin{array}{l}\text { Common, in subalpine and alpine } \\
\text { meadows }\end{array}$ \\
\hline \multicolumn{8}{|c|}{ Polemoniaceae } \\
\hline 189 & Polemonium caeruleum L. & $\mathrm{H}$ & $\begin{array}{l}\text { SPS 3538, } \\
3751,3849\end{array}$ & July-Sep. & $2900-3300$ & $\mathrm{~N}, \mathrm{C}, \mathrm{CT}$ & $\begin{array}{l}\text { Frequent, under Rhododendron- } \\
\text { Betula forests }\end{array}$ \\
\hline \multicolumn{8}{|c|}{ Boraginaceae } \\
\hline 190 & $\begin{array}{l}\text { Cynoglossum microglochin var. } \\
\text { nervosum (Benth. ex C.B. Clarke) } \\
\text { Y.J. Nasir }\end{array}$ & $\mathrm{H}$ & $\begin{array}{l}\text { SPS 3720, } \\
4061\end{array}$ & June-Sep. & $2400-3300$ & $N, C$ & $\begin{array}{l}\text { Frequent, in alpine slopes, below } \\
\text { Chur peak }\end{array}$ \\
\hline 191 & $\begin{array}{l}\text { Hackelia uncinata (Benth.) C.E.C. } \\
\text { Fisch. }\end{array}$ & $\mathrm{H}$ & SPS 3985 & July-Sep. & $2400-3100$ & $\mathrm{~N}, \mathrm{C}, \mathrm{CT}$ & $\begin{array}{l}\text { Common, humus rich soil, under } \\
\text { shrubberies in conifer forests }\end{array}$ \\
\hline 192 & Lindelofia longiflora (Benth.) Baill. & $\mathrm{H}$ & SPS 4254 & July-Sep. & $2600-3300$ & $\mathrm{~N}, \mathrm{C}, \mathrm{CT}$ & Common, in alpine meadows \\
\hline 193 & $\begin{array}{l}\text { Mertensia racemosa Benth. ex C.B. } \\
\text { Clarke }\end{array}$ & $\mathrm{H}$ & SPS 4037 & June-Sep. & $2900-3200$ & $N$ & Frequent, in subalpine scrub \\
\hline 194 & Myosotis sylvatica Ehrh. ex Hoffm. & $\mathrm{H}$ & SPS 3770 & April-Sep. & $2650-3150$ & $\mathrm{~N}, \mathrm{C}, \mathrm{CT}$ & $\begin{array}{l}\text { Common, in shrubberies under } \\
\text { Abies spectabilis forests }\end{array}$ \\
\hline \multicolumn{8}{|c|}{ Solanaceae } \\
\hline 196 & Solanum pseudocapsicum L. & $\mathrm{H}$ & SPS 4375 & July-Sep. & $1900-2200$ & C & $\begin{array}{l}\text { Occasional, along roadsides and } \\
\text { waysides }\end{array}$ \\
\hline \multicolumn{8}{|c|}{ Scrophulariaceae } \\
\hline 196 & Hemiphragma heterophyllum Wall. & $\mathrm{H}$ & $\begin{array}{l}\text { SPS 3524, } \\
4140 \\
\end{array}$ & June-Sep. & $2200-2900$ & $\mathrm{~N}, \mathrm{C}, \mathrm{CT}$ & $\begin{array}{l}\text { Frequent, in shady places under } \\
\text { conifer forests }\end{array}$ \\
\hline 197 & $\begin{array}{l}\text { Leptorhabdos parviflora (Benth.) } \\
\text { Benth. }\end{array}$ & $\mathrm{H}$ & SPS 4536 & July-Oct. & $1900-2300$ & $N$ & $\begin{array}{l}\text { Frequent, at foothills and grazed } \\
\text { areas }\end{array}$ \\
\hline 198 & Pedicularis bicornuta Klotzsch & $\mathrm{H}$ & SPS 3729 & July-Aug. & $3100-3400$ & $N$ & $\begin{array}{l}\text { Frequent, in damp places below } \\
\text { Chur peak }\end{array}$ \\
\hline 199 & Pedicularis gracilis Wall. ex Benth. & $\mathrm{H}$ & \begin{tabular}{|l|} 
SPS 3977, \\
4168 \\
\end{tabular} & July-Sep. & $2800-3100$ & $\mathrm{~N}, \mathrm{C}, \mathrm{CT}$ & $\begin{array}{l}\text { Common, in subalpine slopes under } \\
\text { shrubberies }\end{array}$ \\
\hline 200 & Pedicularis hoffmeisteri Klotzsch & $\mathrm{H}$ & \begin{tabular}{|l} 
SPS 4060, \\
3768,4166 \\
\end{tabular} & July-Sep. & $2900-3500$ & $\mathrm{~N}, \mathrm{C}, \mathrm{CT}$ & $\begin{array}{l}\text { Frequent, in damp places below } \\
\text { Chur peak }\end{array}$ \\
\hline 201 & $\begin{array}{l}\text { Pedicularis longiflora Rudolph var. } \\
\text { tubiformis (Klotzch) Tsoong }\end{array}$ & $\mathrm{H}$ & SPS 4071 & June-Sep. & $3100-3300$ & CT & Rare, in alpine meadows \\
\hline 202 & Pedicularis pectinata Wall. ex Benth. & $\mathrm{H}$ & $\begin{array}{l}\text { SPS 3715, } \\
4059,4136 \\
\end{array}$ & July-Sep. & $2900-3500$ & $\mathrm{~N}, \mathrm{C}, \mathrm{CT}$ & $\begin{array}{l}\text { Common, in open alpine slopes, } \\
\text { damp places }\end{array}$ \\
\hline 203 & Pedicularis punctata Decne. & $\mathrm{H}$ & SPS 4150 & July-Sep. & $3100-3500$ & $N$ & $\begin{array}{l}\text { Common, in alpine slopes, wet } \\
\text { habitats }\end{array}$ \\
\hline 204 & $\begin{array}{l}\text { Pedicularis pyramidata Royle ex } \\
\text { Benth. }\end{array}$ & $\mathrm{H}$ & $\begin{array}{l}\text { SPS 3743, } \\
4137 \\
\end{array}$ & July-Sep. & $3400-3600$ & $\mathrm{~N}, \mathrm{C}, \mathrm{CT}$ & Frequent, under subalpine scrub \\
\hline 205 & $\begin{array}{l}\text { Veronica alpina L. subsp. pumila } \\
\text { (All.) Dostal }\end{array}$ & $\mathrm{H}$ & SPS 3728 & June-Aug. & $2900-3400$ & CT & Common, in subalpine slopes \\
\hline 206 & Veronica biloba $\mathrm{L}$. & $\mathrm{H}$ & SPS 4505 & May-Sep. & $2400-3300$ & $\mathrm{~N}, \mathrm{C}$ & $\begin{array}{l}\text { Common, in moist places, along } \\
\text { water channels }\end{array}$ \\
\hline 207 & Veronica melissifolia Desf. ex Poir. & $\mathrm{H}$ & SPS 4569 & June-Aug. & $2700-3000$ & $\mathrm{~N}, \mathrm{C}, \mathrm{CT}$ & $\begin{array}{l}\text { Common, in subalpine slopes and } \\
\text { shrubberies }\end{array}$ \\
\hline
\end{tabular}




\begin{tabular}{|c|c|c|c|c|c|c|c|}
\hline & Botanical Name & Habit & $\begin{array}{l}\text { HFRI } \\
\text { collection } \\
\text { no. }\end{array}$ & $\begin{array}{l}\text { Flowering \& } \\
\text { fruiting }\end{array}$ & $\begin{array}{l}\text { Altitudinal } \\
\text { range }(\mathrm{m})\end{array}$ & Forest area & Remarks \\
\hline 208 & $\begin{array}{l}\text { Wulfeniopsis amherstiana (Benth.) } \\
\text { D.Y. Hong }\end{array}$ & $\mathrm{H}$ & SPS 4171 & June-Aug. & $2700-3150$ & CT & $\begin{array}{l}\text { Frequent, in humus rich soils under } \\
\text { conifers, Oak forests }\end{array}$ \\
\hline \multicolumn{8}{|c|}{ Orobanchaceae } \\
\hline 209 & Orobanche alba Stephan & $\mathrm{H}$ & SPS 3822 & July-Sep. & $2400-3000$ & $\mathrm{~N}, \mathrm{CT}$ & $\begin{array}{l}\text { Frequent, in humus rich soils under } \\
\text { conifer, Oak forests }\end{array}$ \\
\hline \multicolumn{8}{|c|}{ Gesneriaceae } \\
\hline 210 & Chirita bifolia D. Don & $\mathrm{H}$ & SPS 4286 & July-Sep. & $2200-2500$ & $\mathrm{~N}, \mathrm{C}$ & $\begin{array}{l}\text { Frequent, in damp places at foot } \\
\text { hills }\end{array}$ \\
\hline \multicolumn{8}{|c|}{ Acanthaceae } \\
\hline 211 & Strobilanthes atropurpureus Nees & SS & SPS 3631 & July-Oct. & $2600-2950$ & $\mathrm{~N}, \mathrm{CT}$ & $\begin{array}{l}\text { Common, as undergrowth in the } \\
\text { forests }\end{array}$ \\
\hline 212 & Strobilanthes wallichii Nees & SS & SPS 4546 & July-Oct. & $1900-2700$ & $\mathrm{~N}, \mathrm{C}$ & $\begin{array}{l}\text { Occasional, as undergrowth in Fir } \\
\text { and Kharshu oak forests }\end{array}$ \\
\hline \multicolumn{8}{|c|}{ Lamiaceae } \\
\hline 213 & Elsholtzia ciliata (Thunb.) Hyl. & $\mathrm{H}$ & SPS 3866 & July-Sep. & $2200-2650$ & $\mathrm{~N}, \mathrm{C}, \mathrm{CT}$ & $\begin{array}{l}\text { Common, in waysides and grazed } \\
\text { areas }\end{array}$ \\
\hline 214 & Lamium album $\mathrm{L}$. & $\mathrm{H}$ & SPS 4531 & April-Oct. & $2400-2700$ & $\mathrm{~N}, \mathrm{C}, \mathrm{CT}$ & $\begin{array}{l}\text { Frequent, in waysides and forest } \\
\text { margins }\end{array}$ \\
\hline 215 & Leonurus cardiaca L. & $\mathrm{H}$ & SPS 3789 & June-Sep. & $2100-2800$ & C, CT & Common, in moist and shady places \\
\hline 216 & Nepeta connata Royle ex Benth. & $\mathrm{H}$ & SPS 4548 & July-Sep. & $2900-3300$ & $\mathrm{~N}, \mathrm{C}, \mathrm{CT}$ & Frequent, in subalpine scrub \\
\hline 217 & $\begin{array}{l}\text { Nepeta laevigata (D.Don) Hand.- } \\
\text { Mazz. }\end{array}$ & $\mathrm{H}$ & $\begin{array}{l}\text { SPS 3993, } \\
4143\end{array}$ & July-Oct. & $2900-3150$ & $\mathrm{~N}, \mathrm{C}, \mathrm{CT}$ & $\begin{array}{l}\text { Frequent, in moist and shady } \\
\text { places, forest shrubberies }\end{array}$ \\
\hline 218 & Phlomis macrophylla Benth. & $\mathrm{H}$ & SPS 3625 & July-Sep. & $3400-3640$ & $\mathrm{CT}, \mathrm{C}$ & Rare, in alpine meadows \\
\hline 219 & Scutellaria scandens D. Don & $\mathrm{H}$ & SPS 3773 & April-July & $2300-3050$ & $\mathrm{~N}, \mathrm{C}, \mathrm{CT}$ & $\begin{array}{l}\text { Common, in open slopes, shady } \\
\text { places, shrubberies }\end{array}$ \\
\hline 220 & Stachys melissifolia Benth. & $\mathrm{H}$ & SPS 4566 & July-Sep. & $2200-3150$ & $\mathrm{~N}, \mathrm{C}, \mathrm{CT}$ & $\begin{array}{l}\text { Common, in shady places under } \\
\text { conifer forests }\end{array}$ \\
\hline \multicolumn{8}{|c|}{ Phytolaccaceae } \\
\hline 221 & Phytolacca acinosa Roxb. & SS & $\begin{array}{l}\text { SPS 3796, } \\
3935\end{array}$ & May-Sep. & $2400-2800$ & $\mathrm{CT}, \mathrm{C}$ & $\begin{array}{l}\text { Frequent, in shrubberies under } \\
\text { Deodar forests }\end{array}$ \\
\hline \multicolumn{8}{|c|}{ Polygonaceae } \\
\hline 222 & $\begin{array}{l}\text { Aconogonon rumicifolium (Royle ex } \\
\text { Bab.) H. Hara }\end{array}$ & $\mathrm{H}$ & \begin{tabular}{|l|} 
SPS 3647, \\
3724,3963 \\
\end{tabular} & July-Sep. & $3150-3600$ & $\mathrm{~N}, \mathrm{CT}$ & $\begin{array}{l}\text { Common, in alpine slopes, damp } \\
\text { places }\end{array}$ \\
\hline 223 & Bistorta affinis (D. Don) Greene & $\mathrm{H}$ & SPS 4335 & June-Sep. & $3050-3300$ & $\mathrm{~N}, \mathrm{CT}$ & Common in moist, shady places \\
\hline 224 & Bistorta emodi (Meisn.) H. Hara & $\mathrm{H}$ & SPS 4027 & July-Sep. & $3000-3500$ & $\mathrm{~N}, \mathrm{C}, \mathrm{CT}$ & $\begin{array}{l}\text { Occasional, in moist slopes of } \\
\text { alpine, subalpines }\end{array}$ \\
\hline 225 & Persicaria vivipara (L.) Ronse Decr. & $\mathrm{H}$ & $\begin{array}{l}\text { SPS 3660, } \\
3732\end{array}$ & June-Sep. & $3150-3600$ & $\mathrm{~N}, \mathrm{CT}$ & $\begin{array}{l}\text { Common, in alpine meadows, } \\
\text { below Chur peak }\end{array}$ \\
\hline 226 & Persicaria wallichii Greuter \& Burdet & SS & SPS 4395 & July-Oct. & $3050-3450$ & $\mathrm{~N}, \mathrm{C}, \mathrm{CT}$ & $\begin{array}{l}\text { Common, in wet places, in } \\
\text { degraded areas }\end{array}$ \\
\hline 227 & Polygonum delicatulum Meisn. & $\mathrm{H}$ & SPS 4139 & July-Sep. & $2300-3500$ & $\mathrm{~N}, \mathrm{C}, \mathrm{CT}$ & $\begin{array}{l}\text { Common, in wet rocky slopes, along } \\
\text { water cources }\end{array}$ \\
\hline 228 & Polygonum filicaule Wall. ex Meisn. & $\mathrm{H}$ & SPS 4281 & July-Sep. & $2400-3000$ & $\mathrm{~N}, \mathrm{C}, \mathrm{CT}$ & $\begin{array}{l}\text { Common, in wet rocky slopes, along } \\
\text { water cources }\end{array}$ \\
\hline 229 & $\begin{array}{l}\text { Polygonum paronychioides C.A. } \\
\text { Mey. }\end{array}$ & $\mathrm{H}$ & SPS 4530 & June-Oct. & $2650-2900$ & $\mathrm{~N}, \mathrm{C}, \mathrm{CT}$ & $\begin{array}{l}\text { Common, in wet places, along } \\
\text { watercources }\end{array}$ \\
\hline \multicolumn{8}{|c|}{ Lauraceae } \\
\hline 230 & Neolitsea lanuginosa (Nees) Gamble & ST & SPS 3600 & Mar.-June & $2400-2650$ & C, CT & Occasional, along river beds \\
\hline 231 & Neolitsea umbrosa (Nees) Gamble & $T$ & SPS 4378 & Sep.-Oct. & $2150-2700$ & c & $\begin{array}{l}\text { Occasional, along river beds and in } \\
\text { Ban oak forests }\end{array}$ \\
\hline \multirow[t]{2}{*}{232} & Persea odoratissima (Nees) Kosterm. & $\mathrm{T}$ & $\begin{array}{l}\text { SPS 3596, } \\
3545,3905\end{array}$ & Mar.-July & $1900-2200$ & C, CT & $\begin{array}{l}\text { Occasional, along river beds and in } \\
\text { Ban oak forests }\end{array}$ \\
\hline & Thymelaeaceae & & & & & & \\
\hline \multirow[t]{2}{*}{233} & $\begin{array}{l}\text { Wikstroemia canescens Wall. ex } \\
\text { Meisn. }\end{array}$ & $\mathrm{H}$ & $\begin{array}{l}\text { SPS 3579, } \\
3894 \\
\end{array}$ & July-Sep. & $2550-3150$ & $\mathrm{~N}, \mathrm{C}, \mathrm{CT}$ & $\begin{array}{l}\text { Common, in shady places under } \\
\text { Deodar forests }\end{array}$ \\
\hline & Elaeagnaceae & & & & & & \\
\hline \multirow[t]{2}{*}{234} & Elaeagnus parvifolia Wall. ex Royle & s & SPS 4279 & April-July & $1900-2400$ & $\mathrm{~N}$ & Common, in dry open slopes \\
\hline & Loranthaceae & & & & & & \\
\hline 235 & Scurrula cordifolia G. Don & $P$ & SPS 4427 & Aug.-Oct. & $1900-2300$ & C, CT & $\begin{array}{l}\text { Frequent, on branches of Pyrus, } \\
\text { Prunus and Quercus spp. }\end{array}$ \\
\hline
\end{tabular}




\begin{tabular}{|c|c|c|c|c|c|c|c|}
\hline & Botanical Name & Habit & $\begin{array}{l}\text { HFRI } \\
\text { collection } \\
\text { no. }\end{array}$ & $\begin{array}{l}\text { Flowering \& } \\
\text { fruiting }\end{array}$ & $\begin{array}{l}\text { Altitudinal } \\
\text { range }(\mathrm{m})\end{array}$ & Forest area & Remarks \\
\hline & Euphorbiaceae & & & & & & \\
\hline 236 & Euphorbia maddenii Boiss. & $\mathrm{H}$ & SPS 3548 & May-Aug. & $2150-2400$ & $C T, C$ & $\begin{array}{l}\text { Occasional, in degraded and grazing } \\
\text { areas }\end{array}$ \\
\hline 237 & Euphorbia parviflora L. & H & SPS 4538 & May-Aug. & $1900-2450$ & $\mathrm{~N}, \mathrm{C}, \mathrm{CT}$ & $\begin{array}{l}\text { Common in open slopes, pathways, } \\
\text { forest margins }\end{array}$ \\
\hline \multirow[t]{2}{*}{238} & Euphorbia stracheyi Boiss. & H & $\begin{array}{l}\text { SPS } 3699, \\
3733,4025\end{array}$ & May-June & $2950-3600$ & $\mathrm{~N}, \mathrm{CT}$ & Common in alpine meadows, slopes \\
\hline & Buxaceae & & & & & & \\
\hline \multirow[t]{2}{*}{239} & Buxus wallichiana Baill. & ST & SPS 4354 & Mar.-May & $2100-2500$ & C & $\begin{array}{l}\text { Occasional, along ravines and other } \\
\text { shady places }\end{array}$ \\
\hline & Urticaceae & & & & & & \\
\hline 240 & $\begin{array}{l}\text { Lecanthus peduncularis (Wall. ex } \\
\text { Royle) Wedd. }\end{array}$ & $\mathrm{H}$ & $\begin{array}{l}\text { SPS 3984, } \\
3774\end{array}$ & July-Sep. & $2300-3200$ & $\mathrm{~N}, \mathrm{C}, \mathrm{CT}$ & $\begin{array}{l}\text { Common, in moist rocky crevices, } \\
\text { along water cources }\end{array}$ \\
\hline \multirow[t]{2}{*}{241} & Pouzolzia sanguinea (Blume) Merr. & $\mathrm{H}$ & SPS 4543 & June-Sep. & $1900-2550$ & $\mathrm{~N}, \mathrm{C}, \mathrm{CT}$ & Occasional, on drier slopes \\
\hline & Moraceae & & & & & & \\
\hline 242 & Ficus neriifolia $\mathrm{Sm}$. & $\mathrm{T}$ & SPS 4400 & May-Sep. & $1900-2400$ & $\mathrm{~N}, \mathrm{C}, \mathrm{CT}$ & $\begin{array}{l}\text { Occasional, in Ban oak forests and } \\
\text { in waysides }\end{array}$ \\
\hline 243 & Ficus sarmentosa Buch.-Ham. ex Sm. & $S$ & SPS 4408 & May-Aug. & $2200-2450$ & $\mathrm{~N}, \mathrm{C}, \mathrm{CT}$ & $\begin{array}{l}\text { Common, creeping over rockes } \\
\text { and trees }\end{array}$ \\
\hline \multirow[t]{2}{*}{244} & $\begin{array}{l}\text { Ficus semicordata Buch.-Ham. ex } \\
\text { Sm. }\end{array}$ & ST & SPS 4466 & Aug.-Sep. & $1900-2150$ & C & Occasional, in steep rocky slopes \\
\hline & Juglandaceae & & & & & & \\
\hline \multirow[t]{2}{*}{245} & Juglans regia $\mathrm{L}$. & $\mathrm{T}$ & SPS 4351 & Mar.-Sep. & $2400-2700$ & $N, C$ & $\begin{array}{l}\text { Occasional, planted along field } \\
\text { bunds }\end{array}$ \\
\hline & Betulaceae & & & & & & \\
\hline \multirow[t]{2}{*}{246} & Betula utilis D.Don & $\mathrm{T}$ & SPS 3961 & May-Sep. & $2900-3450$ & $\mathrm{~N}, \mathrm{C}, \mathrm{CT}$ & $\begin{array}{l}\text { Common, along tree-lines, } \\
\text { associated with Kharsu oak and } \\
\text { Rhododenron campanulatum } \\
\text { forests }\end{array}$ \\
\hline & Corylaceae & & & & & & \\
\hline \multirow[t]{2}{*}{247} & Corylus jacquemontii Decne. & $\mathrm{T}$ & $\begin{array}{l}\text { SPS } 3542, \\
3870\end{array}$ & Mar.-Aug. & $2300-2800$ & C, CT & $\begin{array}{l}\text { Occasional, associated with Kharshu } \\
\text { oak forests }\end{array}$ \\
\hline & Salicaceae & & & & & & \\
\hline 248 & Salix alba L. & $\mathrm{T}$ & $\begin{array}{l}\text { SPS } 4085, \\
4275\end{array}$ & April-June & $2150-3150$ & CT & Frequent, along ravines \\
\hline 249 & Salix denticulata Andresson & $\mathrm{s}$ & SPS 3694 & Mar.-June & $2650-3050$ & $\mathrm{~N}, \mathrm{C}$ & Common, along water cources \\
\hline 250 & Salix flagellaris Hultén & SS & $\begin{array}{l}\text { SPS } 3626, \\
3745\end{array}$ & June-Aug. & $2950-3400$ & $N$ & $\begin{array}{l}\text { Common, along water cources, in } \\
\text { grassy alpine slopes }\end{array}$ \\
\hline \multirow[t]{3}{*}{251} & Salix lindleyana Wall. ex Andresson & $\mathrm{s}$ & SPS 4545 & June-July & $2900-3300$ & $\mathrm{~N}, \mathrm{CT}$ & $\begin{array}{l}\text { Occasional, in moist watery slopes } \\
\text { at alpines }\end{array}$ \\
\hline & Monocotyledons & & & & & & \\
\hline & Orchidaceae & & & & & & \\
\hline 252 & Calanthe tricarinata Lindl. & $\mathrm{H}$ & $\begin{array}{l}\text { SPS } 3680, \\
3777\end{array}$ & May-July & $2400-3150$ & $\mathrm{~N}, \mathrm{C}, \mathrm{CT}$ & $\begin{array}{l}\text { Frequent, in humus rich soil under } \\
\text { conifer forests }\end{array}$ \\
\hline 253 & Cephalanthera longifolia (L.) Fritsch & $\mathrm{H}$ & $\begin{array}{l}\text { SPS 3944, } \\
4005\end{array}$ & May-July & $2400-2650$ & $\mathrm{~N}, \mathrm{C}, \mathrm{CT}$ & $\begin{array}{l}\text { Frequent, in shady places among } \\
\text { boulders }\end{array}$ \\
\hline 254 & Cypripedium cordigerum D.Don & $\mathrm{H}$ & SPS 3556 & May-uly & $2300-3150$ & $\mathrm{~N}, \mathrm{CT}$ & Rare, in conifer forests \\
\hline 255 & Cypripedium himalaicum Rolfe & $\mathrm{H}$ & SPS 3602 & June-July & $3450-3600$ & $N$ & $\begin{array}{l}\text { Rare, in alpine meadows below } \\
\text { Chur peak }\end{array}$ \\
\hline 256 & Dactylorhiza hatagirea (D.Don) Soo & H & $\begin{array}{l}\text { SPS } 3571 \\
3747,3883 \\
4054\end{array}$ & June-July & $2900-3450$ & $N$ & $\begin{array}{l}\text { Rare, in subalpine and alpine } \\
\text { meadows }\end{array}$ \\
\hline 257 & Dienia cylindrostachya Lindl. & $\mathrm{H}$ & SPS 4070 & July-Aug. & $2900-3300$ & $\mathrm{~N}, \mathrm{CT}$ & $\begin{array}{l}\text { Frequent, under shrubberies of } \\
\text { Rhododendron forests }\end{array}$ \\
\hline 258 & Epipactis helleborine (L.) Crantz & $\mathrm{H}$ & SPS 3787 & June-Aug. & $2450-2950$ & $\mathrm{~N}, \mathrm{CT}$ & $\begin{array}{l}\text { Rare, under shrubberies of } A \text { bies } \\
\text { spectablis }\end{array}$ \\
\hline 259 & Goodyera biflora (Lindl.) Hook. f. & $\mathrm{H}$ & SPS 3785 & July-Sep. & $2400-3000$ & $\mathrm{~N}, \mathrm{C}, \mathrm{CT}$ & $\begin{array}{l}\text { Common, in humus rich soils under } \\
\text { Deodar forests }\end{array}$ \\
\hline 260 & Goodyera fusca (Lindl.) Hook. f. & $\mathrm{H}$ & SPS 3978 & July-Oct. & $3150-3400$ & $N$ & $\begin{array}{l}\text { Occasional, in moist rock crevices } \\
\text { below Chur peak }\end{array}$ \\
\hline 261 & Goodyera repens (L.) R.Br. & $\mathrm{H}$ & $\begin{array}{l}\text { SPS 3589, } \\
3910\end{array}$ & July-Sep. & $2300-2900$ & $\mathrm{~N}, \mathrm{C}, \mathrm{CT}$ & $\begin{array}{l}\text { Frequent, in moist slopes under } \\
\text { conifer forests }\end{array}$ \\
\hline
\end{tabular}




\begin{tabular}{|c|c|c|c|c|c|c|c|}
\hline & Botanical Name & Habit & $\begin{array}{l}\text { HFRI } \\
\text { collection } \\
\text { no. }\end{array}$ & $\begin{array}{l}\text { Flowering \& } \\
\text { fruiting }\end{array}$ & $\begin{array}{l}\text { Altitudinal } \\
\text { range }(\mathrm{m})\end{array}$ & Forest area & Remarks \\
\hline 262 & $\begin{array}{l}\text { Habenaria commelinifolia (Roxb.) } \\
\text { Wall. ex Lindl. }\end{array}$ & $\mathrm{H}$ & SPS 3879 & Aug.-Sep & $2150-2650$ & $N$ & Occasional, in grasslands \\
\hline 263 & Habenaria marginata Colebr. & $\mathrm{H}$ & $\begin{array}{l}\text { SPS 3854, } \\
4160\end{array}$ & Aug.-Sep. & $2200-2950$ & $N$ & Rare, in grassy slopes \\
\hline 264 & Habenaria pectinata D.Don & $\mathrm{H}$ & SPS 4156 & July-Sep. & $2000-2400$ & $\mathrm{~N}, \mathrm{C}, \mathrm{CT}$ & Frequent, in grassy slopes \\
\hline 265 & Herminium monorchis (L.) R. Br. & $\mathrm{H}$ & SPS 4513 & July-Oct. & $2000-2900$ & $\mathrm{~N}, \mathrm{C}$ & Frequent, in open slopes \\
\hline 266 & Malaxis acuminata D.Don & $\mathrm{H}$ & SPS 3510 & July-Aug & $2150-3200$ & $\mathrm{~N}, \mathrm{C}, \mathrm{CT}$ & $\begin{array}{l}\text { Common, as undergrowth of } \\
\text { Rhododendron forests }\end{array}$ \\
\hline 267 & Malaxis muscifera (Lindl.) Kuntze & $\mathrm{H}$ & $\begin{array}{l}\text { SPS 3738, } \\
3890,3916, \\
4068\end{array}$ & July-Aug & 2900-3300 & $N$ & Rare, under Oaks-conifer forests \\
\hline 268 & Platanthera clavigera Lindl. & $\mathrm{H}$ & SPS 3671 & Aug.-Sep. & $2150-2500$ & $\mathrm{~N}, \mathrm{C}$ & Occasional, under Deodar forests \\
\hline 269 & $\begin{array}{l}\text { Platanthera edgeworthii (Hook. f. ex } \\
\text { Collett) R.K. Gupta }\end{array}$ & $\mathrm{H}$ & SPS 4155 & July-Aug & $2150-2500$ & $\mathrm{CT}, \mathrm{N}$ & Occasional, in open grassy slopes \\
\hline 270 & Ponerorchis chusua (D.Don) Soó & $\mathrm{H}$ & SPS 3736 & July-Aug. & $3400-3600$ & $N$ & Rare, in alpine meadows \\
\hline \multirow[t]{2}{*}{271} & Satyrium nepalense D. Don & $\mathrm{H}$ & $\begin{array}{l}\text { SPS 3506, } \\
3981\end{array}$ & July-Sep. & $2200-2650$ & $\mathrm{~N}, \mathrm{C}, \mathrm{CT}$ & $\begin{array}{l}\text { Frequent, under exposed conifer } \\
\text { forests }\end{array}$ \\
\hline & Zingiberaceae & & & & & & \\
\hline 272 & Roscoea alpina Royle & $\mathrm{H}$ & $\begin{array}{l}\text { SPS 3509, } \\
3646\end{array}$ & June-Aug. & $2900-3400$ & $\mathrm{~N}, \mathrm{CT}$ & $\begin{array}{l}\text { Frequent, on dripping rock } \\
\text { boulders, in moist localities }\end{array}$ \\
\hline \multirow[t]{2}{*}{273} & Roscoea purpurea Sm. & $\mathrm{H}$ & SPS 4330 & June-Sep. & $2150-2450$ & $\mathrm{~N}, \mathrm{C}, \mathrm{CT}$ & Frequent, in open grassy slopes \\
\hline & Iridaceae & & & & & & \\
\hline \multirow[t]{2}{*}{274} & Iris kemaonensis Wall. ex D.Don & $\mathrm{H}$ & SPS 4561 & Apr.-Aug. & $2150-2300$ & C, CT & $\begin{array}{l}\text { Occasional, as patches in grazing } \\
\text { grounds and in subalpine }\end{array}$ \\
\hline & Hypoxidaceae & & & & & & \\
\hline \multirow[t]{2}{*}{275} & Hypoxis aurea Lour. & $\mathrm{H}$ & SPS 3637 & July-Sep. & $1900-2450$ & C, CT & Common, grassy slopes at foothills \\
\hline & Dioscoreaceae & & & & & & \\
\hline 276 & Dioscorea deltoidea Wall. ex Griseb. & Tw & SPS 4193 & May-July & $2150-2400$ & C & $\begin{array}{l}\text { Rare, on shrubberies along forest } \\
\text { margins }\end{array}$ \\
\hline \multirow[t]{2}{*}{277} & $\begin{array}{l}\text { Dioscorea melanophyma Prain \& } \\
\text { Burkill }\end{array}$ & Tw & SPS 3871 & July-Sep. & $2150-2400$ & C, CT & $\begin{array}{l}\text { Rare, on shrubberies along forest } \\
\text { margins }\end{array}$ \\
\hline & Alliaceae & & & & & & \\
\hline 278 & Allium humile Kunth & $\mathrm{H}$ & $\begin{array}{l}\text { SPS } 3648, \\
3702,4006, \\
4026 \\
\end{array}$ & July-Sep. & $3050-3640$ & $\mathrm{~N}, \mathrm{C}$ & Rare, in alpine meadows \\
\hline 279 & Allium victorialis $\mathrm{L}$. & $\mathrm{H}$ & SPS 4017 & July-Sep. & $2800-3000$ & $\mathrm{~N}, \mathrm{CT}$ & Rare, in dripping slopes \\
\hline \multirow[t]{2}{*}{280} & Allium wallichii Kunth & $\mathrm{H}$ & $\begin{array}{l}\text { SPS 3953, } \\
3857,4041\end{array}$ & July-Sep. & $2900-3400$ & $\mathrm{~N}, \mathrm{CT}$ & $\begin{array}{l}\text { Rare, in subalpine meadows, rocky } \\
\text { crevices }\end{array}$ \\
\hline & Liliaceae & & & & & & \\
\hline 281 & $\begin{array}{l}\text { Cardiocrinum giganteum (Wall.) } \\
\text { Makino }\end{array}$ & $\mathrm{H}$ & SPS 4381 & June-Aug. & $2150-2400$ & $\mathrm{~N}, \mathrm{CT}$ & $\begin{array}{l}\text { Rare, damp places under dense } \\
\text { forests }\end{array}$ \\
\hline 282 & $\begin{array}{l}\text { Clintonia udensis var. alpina (Kunth } \\
\text { ex Baker) H. Hara }\end{array}$ & $\mathrm{H}$ & SPS 3886 & July-Sep. & $3150-3400$ & $\mathrm{~N}, \mathrm{CT}$ & $\begin{array}{l}\text { Occasional, damp places under } \\
\text { Betula forests }\end{array}$ \\
\hline 283 & Disporum cantoniense (Lour.) Merr. & $\mathrm{H}$ & SPS 3875 & May-Aug. & $2150-2450$ & $\mathrm{~N}, \mathrm{CT}$ & $\begin{array}{l}\text { Rare, at margins of Mohru oak } \\
\text { forests, slopes }\end{array}$ \\
\hline 284 & Gagea lutea (L.) Ker Gawl. & $\mathrm{H}$ & $\begin{array}{l}\text { SPS 3580, } \\
4012\end{array}$ & Apr.-June & $3050-3500$ & $\mathrm{~N}, \mathrm{C}, \mathrm{CT}$ & Frequent, in alpine meadows \\
\hline 285 & Lloydia serotina (L.) Rchb. & $\mathrm{H}$ & SPS 4013 & May-June & $3050-3500$ & $\mathrm{~N}, \mathrm{C}$ & Occasional, in alpine meadows \\
\hline 286 & Paris polyphylla Sm. & $\mathrm{H}$ & SPS 4272 & June-Aug. & $2900-3150$ & $\mathrm{~N}, \mathrm{CT}$ & $\begin{array}{l}\text { Rare, under shrubberies in dense } \\
\text { forests }\end{array}$ \\
\hline 287 & $\begin{array}{l}\text { Polygonatum cirrhifolium (Wall.) } \\
\text { Royle }\end{array}$ & $\mathrm{H}$ & SPS 3608 & May-June & $2900-3400$ & $\mathrm{~N}, \mathrm{CT}$ & $\begin{array}{l}\text { Rare, under conifers dominated } \\
\text { forests }\end{array}$ \\
\hline \multirow[t]{2}{*}{288} & Polygonatum verticillatum (L.) All. & $\mathrm{H}$ & $\begin{array}{l}\text { SPS } 3713, \\
3887\end{array}$ & June-Sep. & $2450-3500$ & $\mathrm{~N}, \mathrm{C}, \mathrm{CT}$ & $\begin{array}{l}\text { Common, in all type of forest } \\
\text { vegetations }\end{array}$ \\
\hline & Trilliaceae & & & & & & \\
\hline \multirow[t]{2}{*}{289} & Trillium govanianum Wall. ex D.Don & $\mathrm{H}$ & \begin{tabular}{|l} 
SPS 3552, \\
4015 \\
\end{tabular} & May-July & $2300-3400$ & $\mathrm{~N}, \mathrm{C}, \mathrm{CT}$ & $\begin{array}{l}\text { Common, under Rhododendron- } \\
\text { Betula forests, subalpine meadows }\end{array}$ \\
\hline & Smilacaceae & & & & & & \\
\hline 290 & Smilax elegans Wall. ex Kunth & $\mathrm{Cl}$ & SPS 3511 & May-Sep. & $2400-2700$ & $\mathrm{~N}, \mathrm{C}, \mathrm{CT}$ & $\begin{array}{l}\text { Frequent, in forest margins and on } \\
\text { thickets }\end{array}$ \\
\hline
\end{tabular}




\begin{tabular}{|c|c|c|c|c|c|c|c|}
\hline & Botanical Name & Habit & \begin{tabular}{|l} 
HFRI \\
collection \\
no. \\
\end{tabular} & $\begin{array}{l}\text { Flowering \& } \\
\text { fruiting }\end{array}$ & $\begin{array}{l}\text { Altitudinal } \\
\text { range }(\mathrm{m})\end{array}$ & Forest area & Remarks \\
\hline & Haemodoraceae & & & & & & \\
\hline 291 & $\begin{array}{l}\text { Aletris pauciflora (Klotzsch) Hand.- } \\
\text { Mazz. }\end{array}$ & $\mathrm{H}$ & $\begin{array}{l}\text { SPS 3707, } \\
3731,3850\end{array}$ & July-Aug. & $3150-3600$ & $\mathrm{~N}, \mathrm{C}, \mathrm{CT}$ & Common, in alpine meadows \\
\hline \multirow[t]{2}{*}{292} & Ophiopogon intermedius D.Don & $\mathrm{H}$ & SPS 3633 & May-Nov. & $2000-2300$ & $\mathrm{~N}, \mathrm{C}, \mathrm{CT}$ & Common, in open grasslands. \\
\hline & Juncaceae & & & & & & \\
\hline 293 & Juncus concinnus D.Don & $\mathrm{H}$ & SPS 3661 & June-Sep. & $2650-3300$ & $\mathrm{~N}, \mathrm{C}, \mathrm{CT}$ & $\begin{array}{l}\text { Common, in alpine meadows, } \\
\text { marshy habitats }\end{array}$ \\
\hline 294 & Juncus himalensis Klotzsch & $\mathrm{H}$ & $\begin{array}{l}\text { SPS 3712, } \\
4125\end{array}$ & June-Oct. & $3050-3500$ & $\mathrm{~N}, \mathrm{C}, \mathrm{CT}$ & $\begin{array}{l}\text { Common, in alpine meadows, } \\
\text { marshy habitats }\end{array}$ \\
\hline \multirow[t]{2}{*}{295} & Juncus thomsonii Buchen & $\mathrm{H}$ & $\begin{array}{l}\text { SPS 3754, } \\
4126 \\
\end{array}$ & June-Sep. & $2700-3400$ & $\mathrm{~N}, \mathrm{C}, \mathrm{CT}$ & $\begin{array}{l}\text { Common, in alpine meadows, } \\
\text { marshy habitats }\end{array}$ \\
\hline & Araceae & & & & & & \\
\hline 296 & Arisaema flavum (Forssk.) Schott & $\mathrm{H}$ & SPS 4329 & June-Sep. & $1900-2950$ & $\mathrm{~N}, \mathrm{C}, \mathrm{CT}$ & Common, in exposed sunny places \\
\hline 297 & Arisaema intermedium Blume & $\mathrm{H}$ & SPS 3821 & June-Aug. & 2300-3050 & $\mathrm{N}, \mathrm{C}$ & Rare, in shady places \\
\hline 298 & Arisaema utile Hook. f. ex Engl. & $\mathrm{H}$ & SPS 3537 & June-Aug. & $2800-3150$ & $\mathrm{~N}, \mathrm{CT}$ & $\begin{array}{l}\text { Occasional, in conifer dominated } \\
\text { forests }\end{array}$ \\
\hline \multirow[t]{2}{*}{299} & $\begin{array}{l}\text { Remusatia pumila (D. Don) H. Li \& } \\
\text { A.Hay }\end{array}$ & $\mathrm{H}$ & SPS 4405 & June-July & $1900-2450$ & $\mathrm{~N}, \mathrm{C}, \mathrm{CT}$ & $\begin{array}{l}\text { Frequent, rock crevices and in shady } \\
\text { slopes }\end{array}$ \\
\hline & Alismataceae & & & & & & \\
\hline \multirow[t]{2}{*}{300} & Alisma plantago-aquatica L. & $\mathrm{H}$ & SPS 4159 & May-Sep. & $2300-2550$ & $\mathrm{~N}, \mathrm{C}$ & Common, in marshy places \\
\hline & Cyperaceae & & & & & & \\
\hline 301 & Carex norvegica Retz. & $\mathrm{H}$ & SPS 3826 & June-Aug. & $2900-3400$ & $\mathrm{~N}, \mathrm{C}, \mathrm{CT}$ & Common, in alpine meadows \\
\hline 302 & Carex filicina Nees & $\mathrm{H}$ & SPS 4463 & June-Aug. & $2150-2900$ & $\mathrm{~N}, \mathrm{C}, \mathrm{CT}$ & $\begin{array}{l}\text { Common, in marshy and open } \\
\text { grassy slopes }\end{array}$ \\
\hline 303 & Carex wallichiana Spreng. & $\mathrm{H}$ & SPS 3833 & June-July & $2200-2900$ & $\mathrm{~N}, \mathrm{C}, \mathrm{CT}$ & $\begin{array}{l}\text { Common, in shady places, forest } \\
\text { margins }\end{array}$ \\
\hline 304 & Carex haematostoma Nees & $\mathrm{H}$ & SPS 4149 & June-Aug. & 2400-3000 & $\mathrm{N}, \mathrm{C}, \mathrm{CT}$ & Common, in open slopes \\
\hline 305 & $\begin{array}{l}\text { Carex hebecarpa C.A. Mey. subsp. } \\
\text { ligulata (Nees) T. Koyama }\end{array}$ & $\mathrm{H}$ & SPS 4464 & June-Sep. & $3150-3400$ & $\mathrm{~N}, \mathrm{C}, \mathrm{CT}$ & Common, in marshy places \\
\hline 306 & Carex nivalis Boott & $\mathrm{H}$ & SPS 3282 & June-Aug. & 2900-3370 & $\mathrm{N}, \mathrm{C}, \mathrm{CT}$ & Occasional, in alpine slopes \\
\hline 307 & Carex obscura Nees & $\mathrm{H}$ & \begin{tabular}{|l} 
SPS 3813, \\
3827 \\
\end{tabular} & July-Sep. & 2400-3000 & $\mathrm{N}, \mathrm{C}, \mathrm{CT}$ & Frequent, in marshy places \\
\hline 308 & Carex remota $\mathrm{L}$. & $\mathrm{H}$ & SPS 3836 & July-Sep. & $2900-3400$ & $\mathrm{~N}, \mathrm{C}, \mathrm{CT}$ & Occasional, in alpine slopes \\
\hline 309 & Cyperus niveus Retz. & $\mathrm{H}$ & SPS 4268 & May-Sep. & $2400-2900$ & $\mathrm{~N}, \mathrm{C}, \mathrm{CT}$ & Common, in grasslands \\
\hline 310 & Eleocharis congesta D. Don & $\mathrm{H}$ & SPS 4270 & Apr.-Aug. & 2900-3350 & $\mathrm{N}, \mathrm{C}, \mathrm{CT}$ & Common, in damp places \\
\hline 311 & $\begin{array}{l}\text { Eleocharis palustris (L.) Roem. \& } \\
\text { Schult. }\end{array}$ & $\mathrm{H}$ & SPS 3815 & May-July & $3050-3350$ & $\mathrm{~N}, \mathrm{C}, \mathrm{CT}$ & Common, in damp places \\
\hline 312 & $\begin{array}{l}\text { Kobresia capillifolia (Decne.) C.B. } \\
\text { Clarke }\end{array}$ & $\mathrm{H}$ & SPS 4131 & July-Sep. & $2900-3450$ & $\mathrm{~N}, \mathrm{CT}$ & Common, in alpine meadows \\
\hline 313 & Kobresia duthiei C.B. Clarke & $\mathrm{H}$ & \begin{tabular}{|l} 
SPS 3709, \\
3737 \\
\end{tabular} & July-Sep. & $3400-3640$ & $N$ & Frequent in alpine meadows \\
\hline 314 & Kobresia laxa Nees & $\mathrm{H}$ & SPS 3693 & June-Aug. & $2900-3640$ & $\mathrm{~N}, \mathrm{C}$ & Frequent, in alpine meadows \\
\hline 315 & Kobresia nepalensis (Nees) Kük. & $\mathrm{H}$ & SPS 4129 & June-Sep. & $2900-3600$ & $\mathrm{~N}, \mathrm{C}, \mathrm{CT}$ & Frequent, in alpine meadows \\
\hline 316 & $\begin{array}{l}\text { Kobresia pygmaea (C.B. Clarke) C.B. } \\
\text { Clarke }\end{array}$ & $\mathrm{H}$ & SPS 3830 & July-Aug. & $2900-3600$ & $\mathrm{~N}, \mathrm{C}, \mathrm{CT}$ & Frequent, in alpine meadows \\
\hline \multirow[t]{2}{*}{317} & $\begin{array}{l}\text { Schoenoplectiella juncoides (Roxb.) } \\
\text { Lye }\end{array}$ & $\mathrm{H}$ & SPS 4541 & June-Sep. & $2000-2600$ & $\mathrm{~N}, \mathrm{C}, \mathrm{CT}$ & $\begin{array}{l}\text { Common, in damp places, along } \\
\text { water bodies }\end{array}$ \\
\hline & Poaceae & & & & & & \\
\hline 318 & Agrostis munroana Aitch. \& Hemsl. & $\mathrm{H}$ & SPS 4118 & June-Sep. & $2800-3250$ & $\mathrm{~N}, \mathrm{C}, \mathrm{CT}$ & $\begin{array}{l}\text { Common, in moist slopes, } \\
\text { subalpine, alpine meadows }\end{array}$ \\
\hline 319 & Agrostis stolonifera $\mathrm{L}$. & $\mathrm{H}$ & SPS 3824 & July-Sep. & $2400-3400$ & $\mathrm{~N}, \mathrm{C}, \mathrm{CT}$ & $\begin{array}{l}\text { Common, in moist slopes, } \\
\text { subalpine, alpine meadows }\end{array}$ \\
\hline 320 & Arundinella nepalensis Trin. & $\mathrm{H}$ & $\begin{array}{l}\text { SPS 4406, } \\
4102\end{array}$ & Aug.-Nov. & $1900-2900$ & $\mathrm{~N}, \mathrm{C}, \mathrm{CT}$ & $\begin{array}{l}\text { Frequent, in shady places, grassy } \\
\text { slopes }\end{array}$ \\
\hline 321 & Brachiaria villosa (Lam.) A. Camus & $\mathrm{H}$ & SPS 4575 & Aug.-Nov. & $1900-2950$ & $\mathrm{~N}, \mathrm{C}, \mathrm{CT}$ & Frequent, along pathways \\
\hline 322 & Bromus ramosus Huds. & $\mathrm{H}$ & $\begin{array}{l}\text { SPS 4109, } \\
4169\end{array}$ & July-Sep. & $2900-3200$ & $\mathrm{~N}, \mathrm{C}, \mathrm{CT}$ & Frequent, in alpine slopes \\
\hline 323 & Calamagrostis emodensis Griseb. & $\mathrm{H}$ & $\begin{array}{l}\text { SPS } 3755 \\
3758,4146\end{array}$ & July-Sep. & $2650-3600$ & $\mathrm{~N}, \mathrm{C}, \mathrm{CT}$ & $\begin{array}{l}\text { Common, in alpine and subalpines, } \\
\text { rocky slopes }\end{array}$ \\
\hline
\end{tabular}




\begin{tabular}{|c|c|c|c|c|c|c|c|}
\hline & Botanical Name & Habit & $\begin{array}{l}\text { HFRI } \\
\text { collection } \\
\text { no. }\end{array}$ & $\begin{array}{l}\text { Flowering \& } \\
\text { fruiting }\end{array}$ & $\begin{array}{l}\text { Altitudinal } \\
\text { range }(\mathrm{m})\end{array}$ & Forest area & Remarks \\
\hline 324 & $\begin{array}{l}\text { Calamagrostis pseudophragmites } \\
\text { (Haller) Koeler }\end{array}$ & $\mathrm{H}$ & $\begin{array}{l}\text { SPS } 3654, \\
3756,4145, \\
4154\end{array}$ & July-Sep. & $3150-3600$ & $\mathrm{~N}, \mathrm{C}, \mathrm{CT}$ & $\begin{array}{l}\text { Common, in alpine and subalpines, } \\
\text { rocky slopes }\end{array}$ \\
\hline 325 & Chrysopogon serrulatus Trin. & $\mathrm{H}$ & SPS 4112 & July-Sep. & $1900-2650$ & $\mathrm{~N}, \mathrm{C}, \mathrm{CT}$ & $\begin{array}{l}\text { Common, in forest margins, } \\
\text { grasslands. }\end{array}$ \\
\hline 326 & Cynodon dactylon (L.) Pers. & H & SPS 3831 & Mar.-Oct. & $1900-2550$ & $\mathrm{~N}, \mathrm{C}, \mathrm{CT}$ & $\begin{array}{l}\text { Common, along roadsides and in } \\
\text { cultivated fields }\end{array}$ \\
\hline 327 & Digitaria cruciata (Nees) A. Camus & $\mathrm{H}$ & SPS 3651 & July-Oct. & $2150-2900$ & $\mathrm{~N}, \mathrm{C}, \mathrm{CT}$ & $\begin{array}{l}\text { Common, shady places along } \\
\text { watercourses }\end{array}$ \\
\hline 328 & Echinochloa colona (L.) Link & $\mathrm{H}$ & SPS 4165 & Mar.-Oct. & $1900-2200$ & $\mathrm{~N}, \mathrm{C}, \mathrm{CT}$ & $\begin{array}{l}\text { Common, in moist places, waysides } \\
\text { and rocky slopes }\end{array}$ \\
\hline 329 & Echinochloa crusgalli (L.) P. Beauv. & H & SPS 4585 & July-Nov. & $1900-2200$ & $\mathrm{~N}, \mathrm{C}, \mathrm{CT}$ & $\begin{array}{l}\text { Frequent, in moist places, } \\
\text { roadsides, foothills }\end{array}$ \\
\hline 330 & Eragrostis pilosa (L.) P. Beauv. & $\mathrm{H}$ & SPS 4328 & Mar.-Oct. & $1900-2650$ & $\mathrm{~N}, \mathrm{C}, \mathrm{CT}$ & Common, in open rocky slopes \\
\hline 331 & Festuca kashmiriana Stapf & $\mathrm{H}$ & SPS 4389 & June-Aug. & $2900-3600$ & $\mathrm{~N}, \mathrm{C}, \mathrm{CT}$ & $\begin{array}{l}\text { Common, in alpine and subalpine } \\
\text { grassy slopes }\end{array}$ \\
\hline 332 & $\begin{array}{l}\text { Festuca valesiaca Schleich. ex } \\
\text { Gaudin }\end{array}$ & $\mathrm{H}$ & $\begin{array}{l}\text { SPS } 3761 \\
3829,4153\end{array}$ & June-Sep. & $2900-3600$ & $\mathrm{~N}, \mathrm{C}, \mathrm{CT}$ & $\begin{array}{l}\text { Common, in alpine and subalpine } \\
\text { grassy slopes }\end{array}$ \\
\hline 333 & $\begin{array}{l}\text { Muhlenbergia himalayensis Hack. } \\
\text { ex Hook. f. }\end{array}$ & $\mathrm{H}$ & SPS 3946 & July-Oct. & $3450-3640$ & $\mathrm{C}, \mathrm{N}$ & Occasional, in alpine grassy slopes \\
\hline 334 & $\begin{array}{l}\text { Phacelurus speciosus (Steud.) C.E. } \\
\text { Hubb. }\end{array}$ & $\mathrm{H}$ & SPS 3760 & July-Sep. & $2150-2400$ & $\mathrm{~N}, \mathrm{CT}$ & Occasional, in shady places \\
\hline 335 & Phleum alpinum $\mathrm{L}$. & $\mathrm{H}$ & $\begin{array}{l}\text { SPS 3757, } \\
4121,4130\end{array}$ & June-Oct. & 2950-3600 & $\mathrm{N}, \mathrm{C}, \mathrm{CT}$ & Common, in alpine meadows \\
\hline 336 & Phleum himalaicum Mez. & $\mathrm{H}$ & $\begin{array}{l}\text { SPS 3807, } \\
3840\end{array}$ & June-Sep. & $2900-3600$ & $\mathrm{~N}, \mathrm{C}$ & $\begin{array}{l}\text { Common, in rocky crevices, alpine } \\
\text { meadows }\end{array}$ \\
\hline 337 & Poa alpina $\mathrm{L}$. & $\mathrm{H}$ & SPS 4105 & June-Sep. & $2900-3600$ & $\mathrm{~N}, \mathrm{C}, \mathrm{CT}$ & Common, in alpine grassy slopes \\
\hline 338 & Poa nemoralis $\mathrm{L}$. & $\mathrm{H}$ & $\begin{array}{l}\text { SPS 3762, } \\
4104\end{array}$ & May-Aug. & $2900-3600$ & $\mathrm{~N}, \mathrm{C}, \mathrm{CT}$ & Common, in alpine grassy slopes \\
\hline 339 & Poa stapfiana Bor & $\mathrm{H}$ & $\begin{array}{l}\text { SPS } 3759, \\
4108\end{array}$ & July-Aug. & 2900-3600 & $\mathrm{N}, \mathrm{C}, \mathrm{CT}$ & $\begin{array}{l}\text { Frequent, in alpines below Chur } \\
\text { peak }\end{array}$ \\
\hline 340 & Saccharum rufipilum Steud. & $\mathrm{H}$ & SPS 4113 & June-Sep. & $2000-2400$ & $\mathrm{~N}, \mathrm{C}, \mathrm{CT}$ & Frequent, in rocky slopes at foothills \\
\hline 341 & Saccharum spontaneum L & $\mathrm{H}$ & SPS 4091 & Aug.-Oct. & $1900-2100$ & $\mathrm{~N}, \mathrm{C}$ & Occasional, along rivulets \\
\hline 342 & Setaria palmifolia (J. Koenig) Stapf & $\mathrm{H}$ & SPS 4471 & Aug.-Oct. & $2200-2450$ & $\mathrm{~N}, \mathrm{C}, \mathrm{CT}$ & $\begin{array}{l}\text { Common, in moist places along } \\
\text { rivulets }\end{array}$ \\
\hline 343 & $\begin{array}{l}\text { Setaria pumila (Poir.) Roem. \& } \\
\text { Schult. }\end{array}$ & $\mathrm{H}$ & SPS 4162 & July-Sep. & $1900-2650$ & $\mathrm{~N}, \mathrm{C}, \mathrm{CT}$ & Frequent, in moist, shady places \\
\hline 344 & $\begin{array}{l}\text { Sinarundinaria falcata (Nees) C.S. } \\
\text { Chao \& Renvoize }\end{array}$ & S & SPS 4195 & June-July & $2000-2800$ & $\mathrm{~N}, \mathrm{C}, \mathrm{CT}$ & Rare, in steep slopes, along nallahs \\
\hline 345 & $\begin{array}{l}\text { Thamnocalamus spathiflorus (Trin.) } \\
\text { Munro }\end{array}$ & S & SPS 4200 & July-Aug. & $2850-3150$ & $\mathrm{~N}, \mathrm{CT}$ & $\begin{array}{l}\text { Rare, in steep slopes of midhills, } \\
\text { below subalpines }\end{array}$ \\
\hline 346 & $\begin{array}{l}\text { Trisetum aeneum (Hook.f.) R.R. } \\
\text { Stewart }\end{array}$ & $\mathrm{H}$ & $\begin{array}{l}\text { SPS 4106, } \\
4117\end{array}$ & June-Aug. & $2150-3400$ & $\mathrm{~N}, \mathrm{C}, \mathrm{CT}$ & $\begin{array}{l}\text { Common, in alpine slopes below } \\
\text { Chur peak }\end{array}$ \\
\hline 347 & $\begin{array}{l}\text { Trisetum clarkei (Hook.f.) R.R. } \\
\text { Stewart }\end{array}$ & $\mathrm{H}$ & SPS 4110 & July-Oct. & $3150-3640$ & $\mathrm{~N}, \mathrm{C}, \mathrm{CT}$ & $\begin{array}{l}\text { Common, in alpine slopes below } \\
\text { Chur peak }\end{array}$ \\
\hline \multirow[t]{3}{*}{348} & Trisetum spicatum (L.) K. Richt. & $\mathrm{H}$ & $\begin{array}{l}\text { SPS 4147, } \\
4163\end{array}$ & July-Aug. & $3150-3640$ & $\mathrm{~N}, \mathrm{C}, \mathrm{CT}$ & $\begin{array}{l}\text { Common, in alpine slopes below } \\
\text { Chur peak }\end{array}$ \\
\hline & Gymnosperms & & & & & & \\
\hline & Pinaceae & & & & & & \\
\hline 349 & Abies spectabilis (D. Don) Mirb. & $\mathrm{T}$ & $\begin{array}{l}\text { SPS 3563, } \\
4023\end{array}$ & May-July & $2900-3400$ & $\mathrm{~N}, \mathrm{C}, \mathrm{CT}$ & Common, in subalpine slopes \\
\hline 350 & Picea smithiana (Wall.) Boiss. & $\mathrm{T}$ & SPS 4265 & Sep.-Oct. & $2200-3300$ & $\mathrm{~N}, \mathrm{C}, \mathrm{CT}$ & Common \\
\hline \multirow[t]{2}{*}{351} & Pinus wallichiana A.B. Jacks. & $\mathrm{T}$ & SPS 4266 & Sep.-Oct. & 1900-3000 & $\mathrm{N}, \mathrm{C}, \mathrm{CT}$ & Common \\
\hline & Taxaceae & & & & & & \\
\hline 352 & Taxus wallichiana Zucc. & ST & SPS 3557 & Mar.-May & $2600-3000$ & C, CT & Occasional \\
\hline
\end{tabular}

T - Trees; ST - Small trees; S - Shrub; SS - Subshrub; U - Undershrub; Cl - Climber; Tw - Twiner; P - Parasites; H - Herbs, N - Nauradhar beat; CT - Chhog Tali beat; C - Churas beat; SPS - S.P. Subramani 


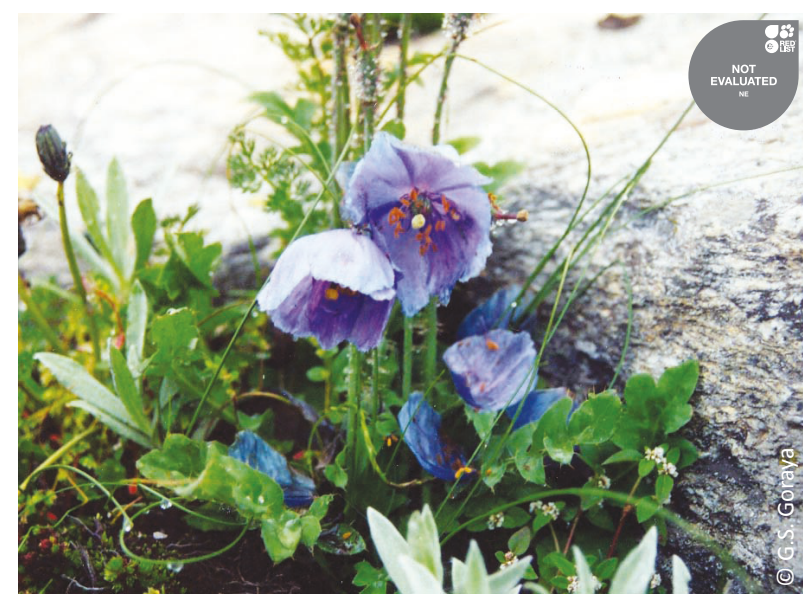

Image 1. Meconopsis aculeata Royle

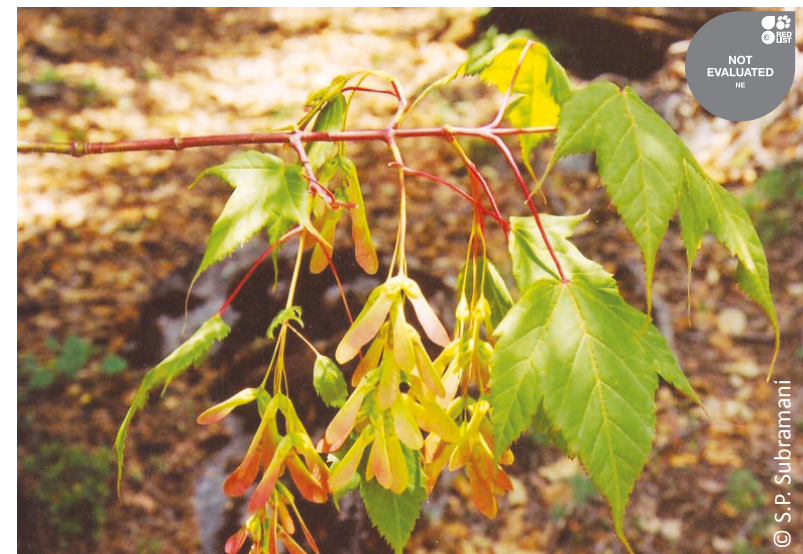

Image 3. Acer acuminatum Wall. ex D.Don

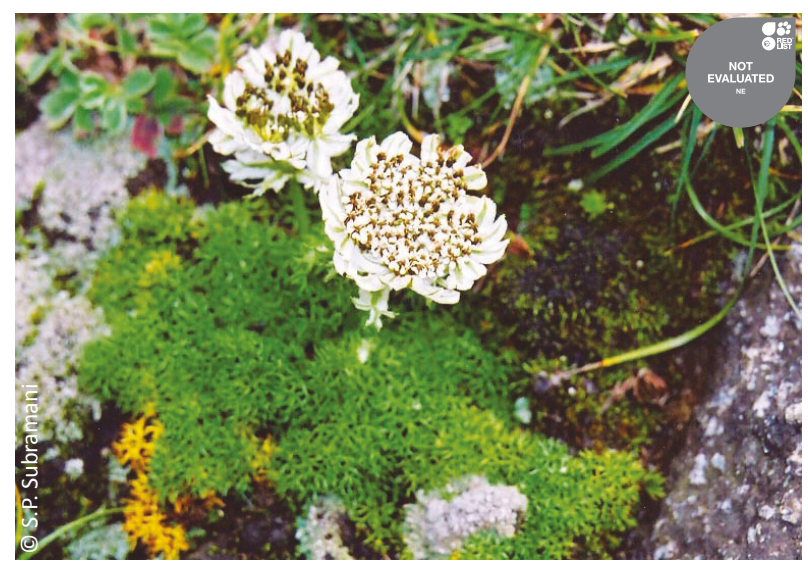

Image 5. Pleurospermum brunonis Benth. ex C.B. Clarke

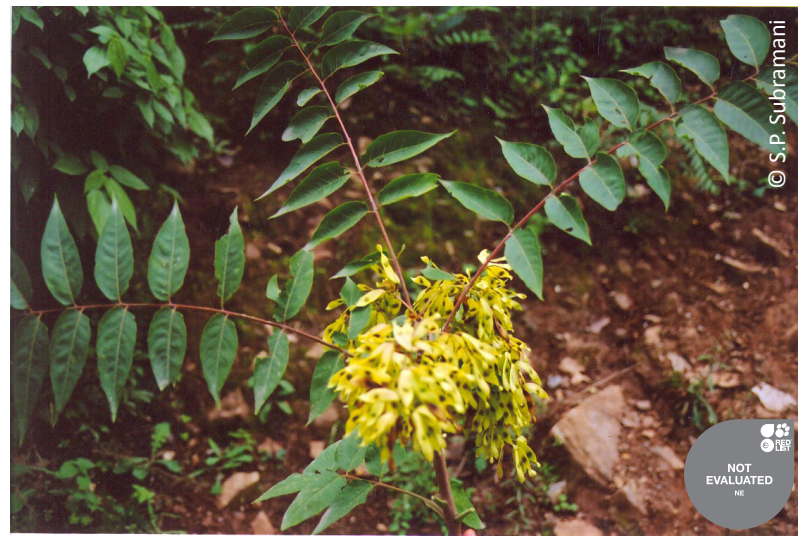

Imgage 2. Ailanthus altissima (Mill.) Swingle

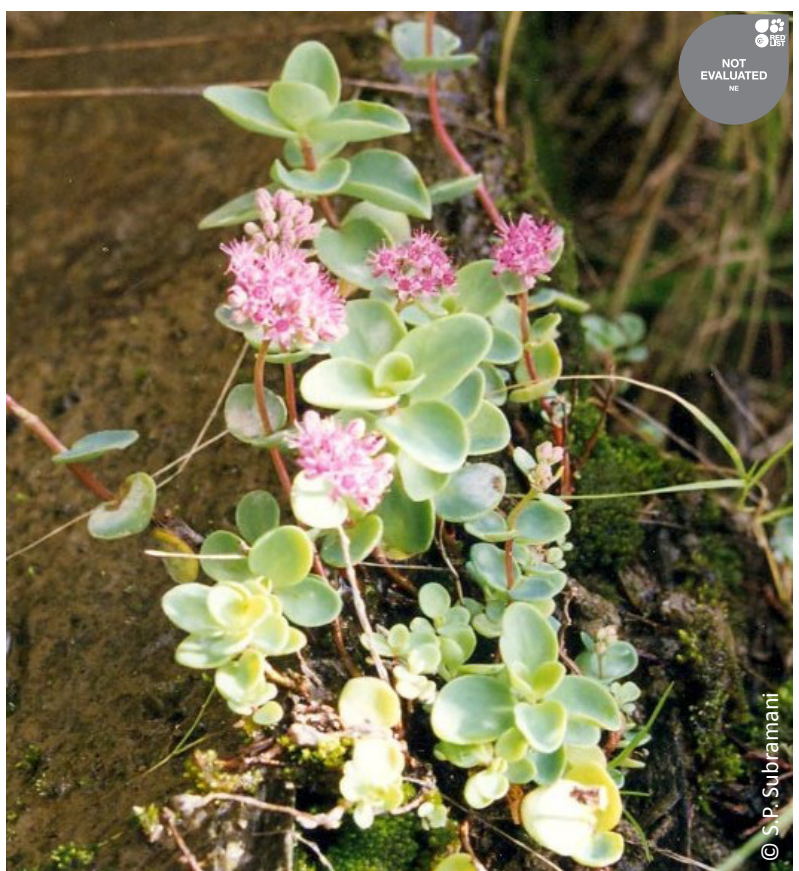

Image 4. Hylotelephium ewersii (Ledeb.) H. Ohbi

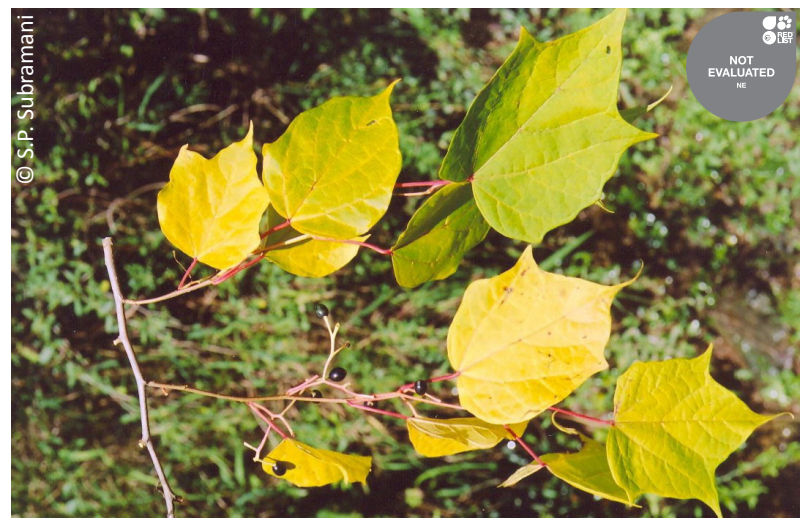

Image 6. Alangium chinense (Lour.) Harms 


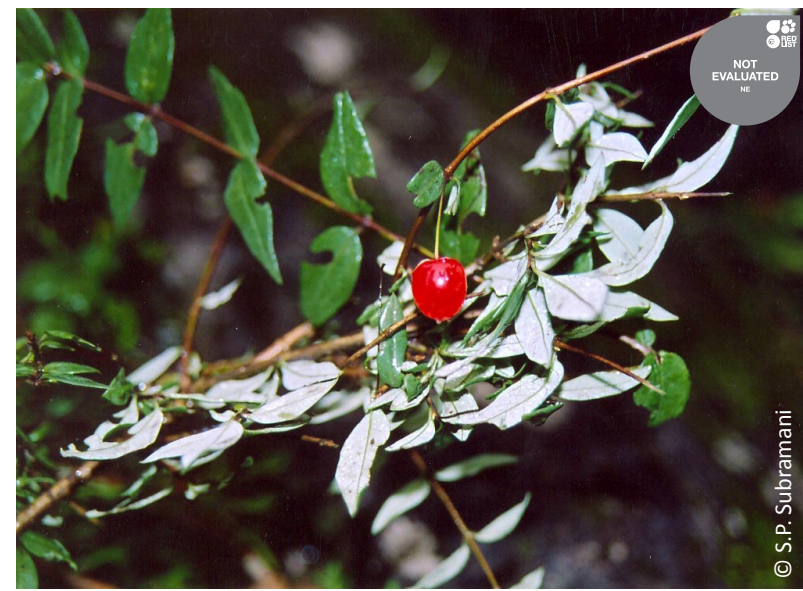

Image 7. Lonicera angustifolia Wall. ex DC.

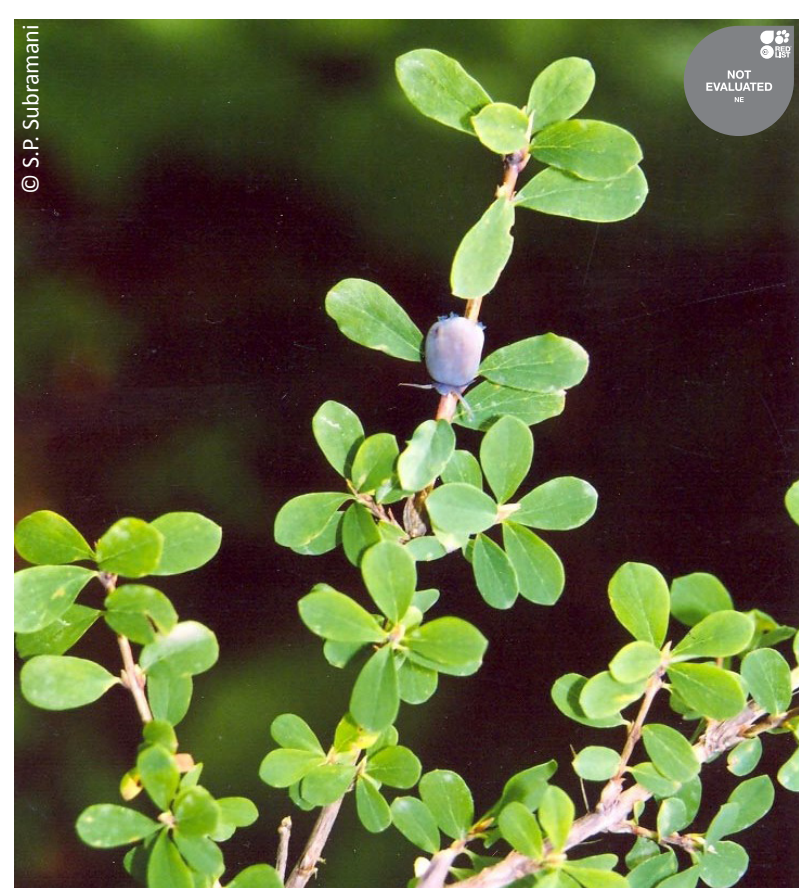

Image 9. Lonicera obovata Royle

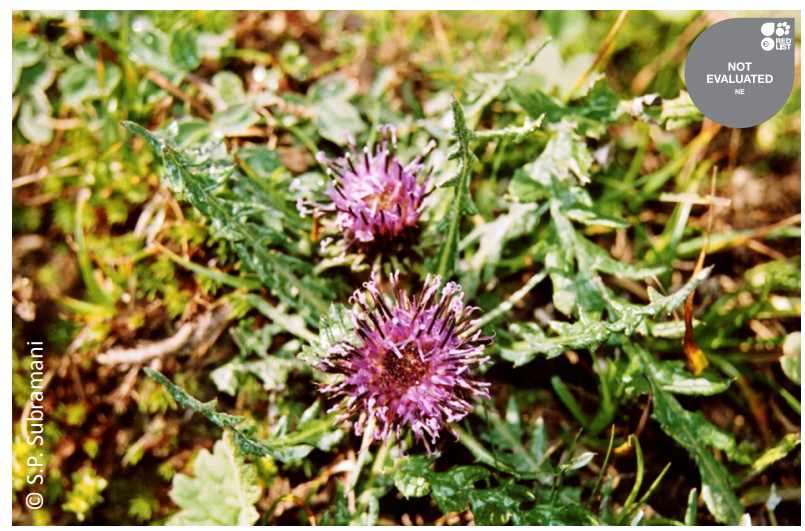

Image 11. Saussurea taraxacifolia Wall. ex DC.

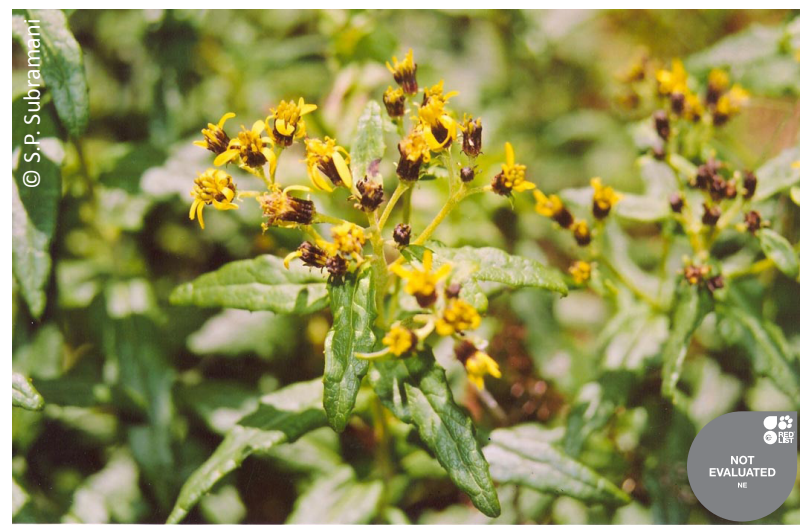

Image 8. Senecio kunthianus Wall. ex DC.

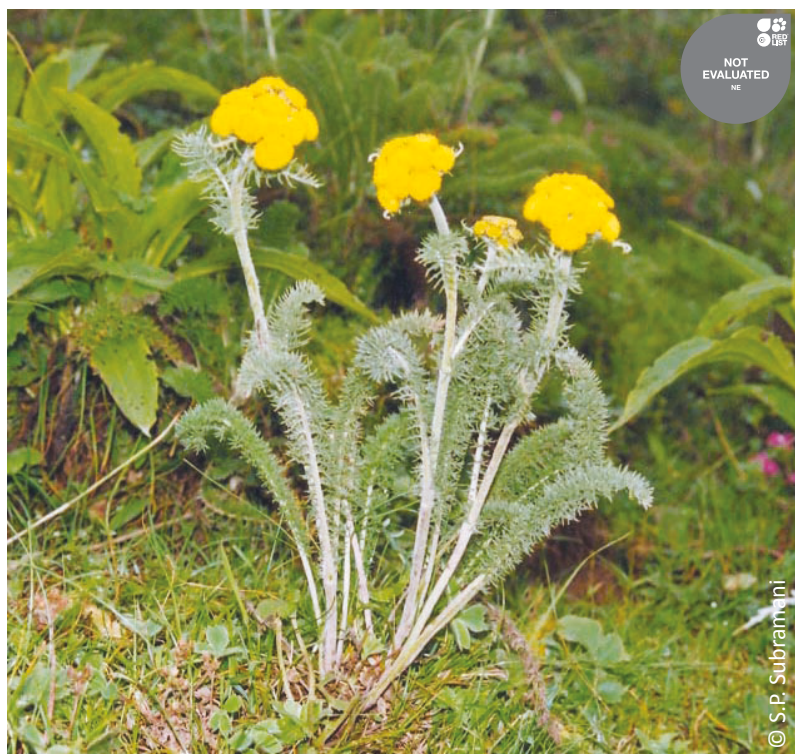

Image 10. Tanacetum longifolium Wall. ex DC.

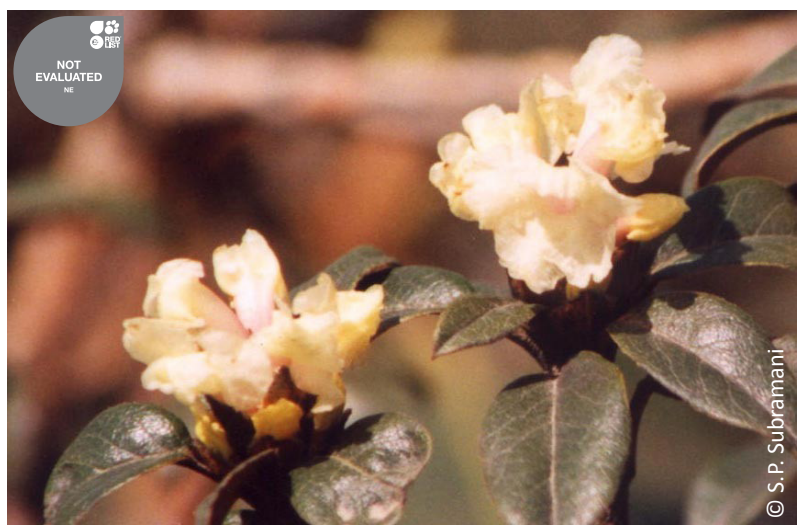

Image 12. Rhododendron anthopogon D.Don 


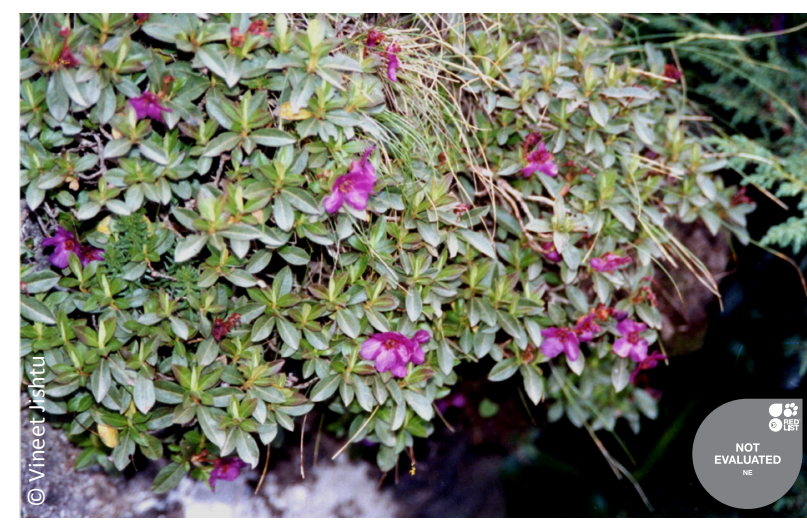

Image 13. Rhododendron lepidotum Wall. ex G.Don

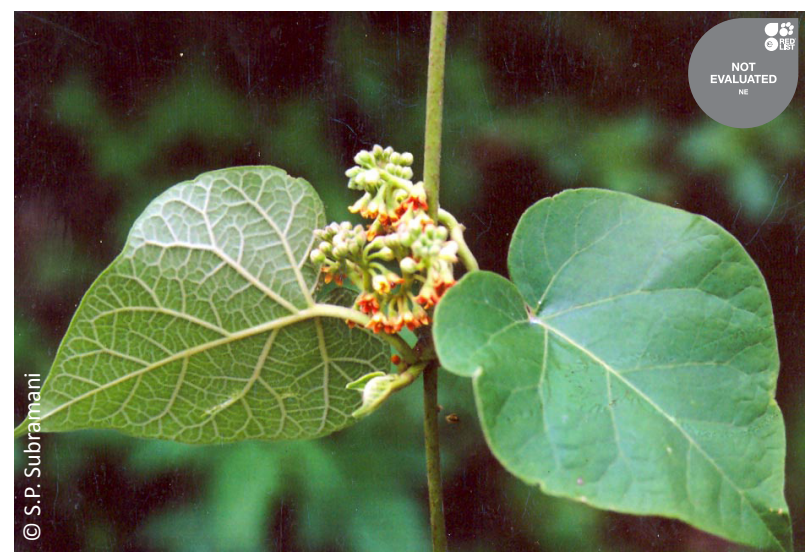

Image 15. Pergularia roylei (Wight) D. Dietr.

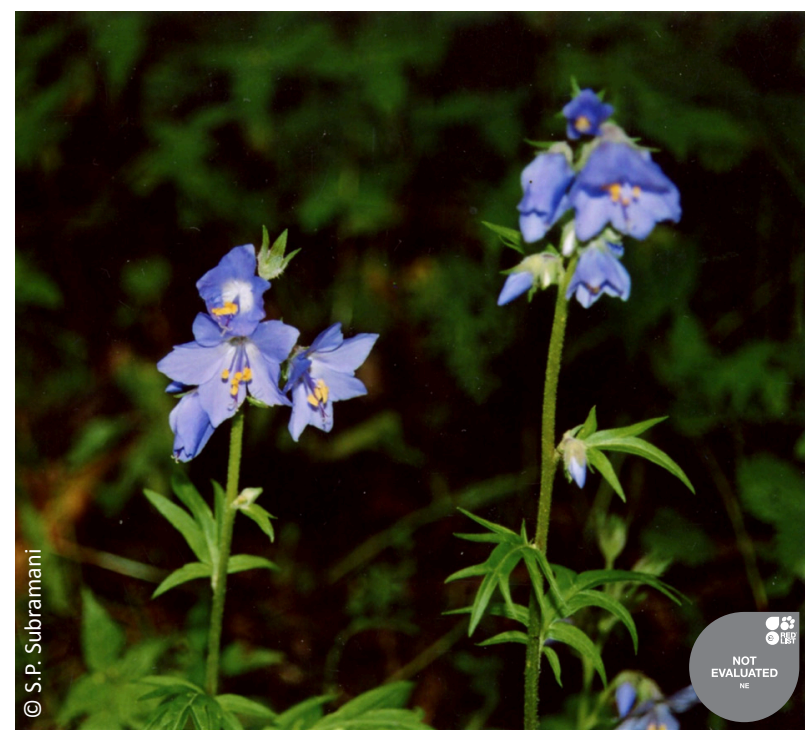

Image 16. Polemonium caeruleum L.

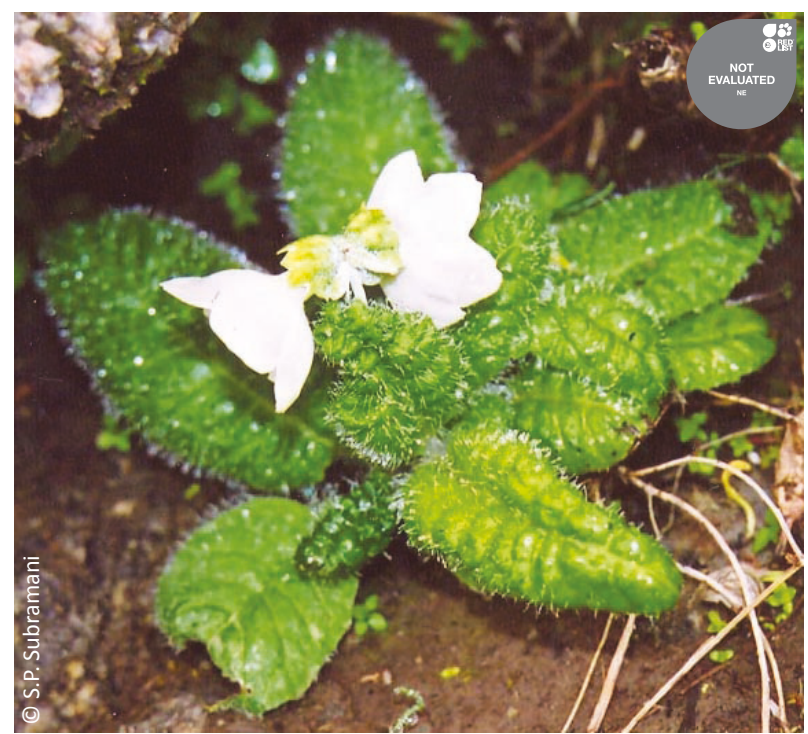

Image 14. Primula reidii Duthie

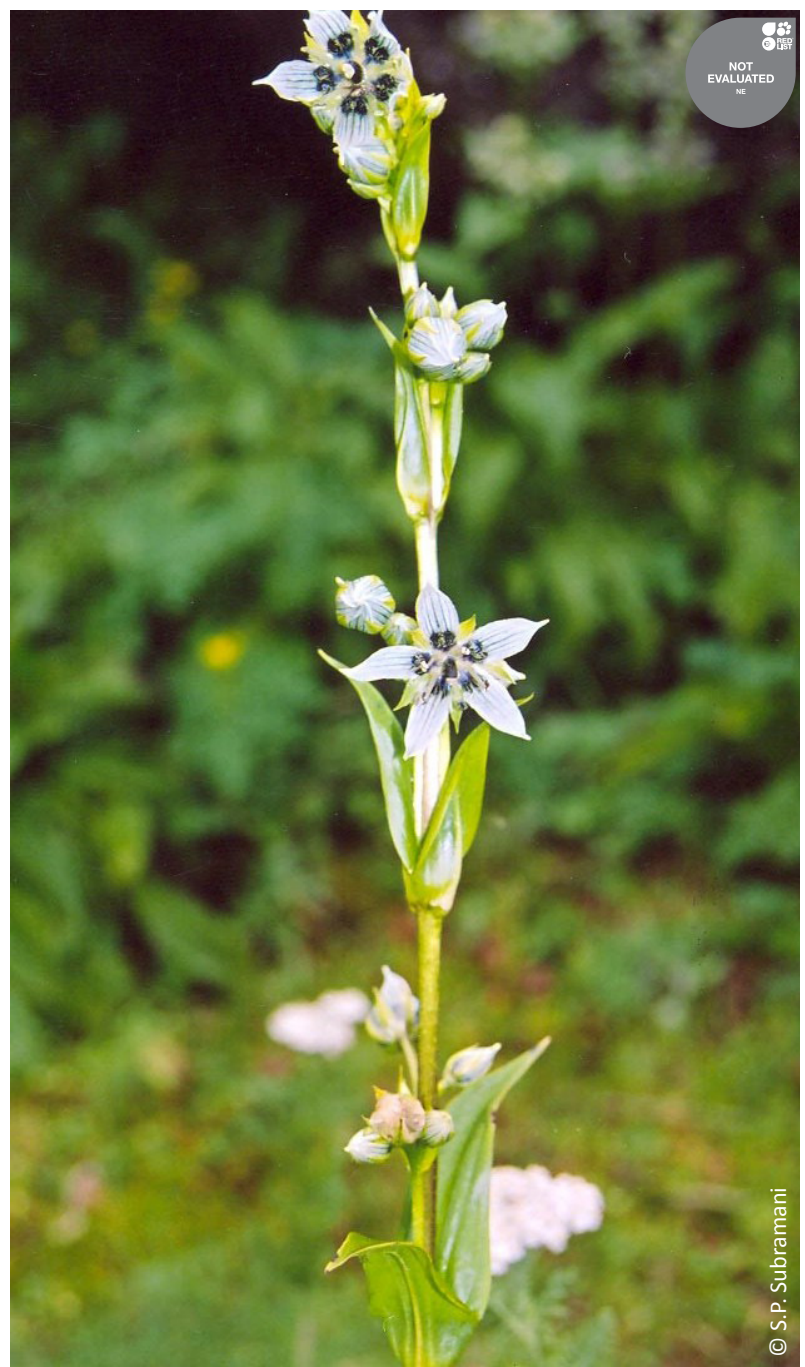

Image 17. Swertia speciosa D.Don 


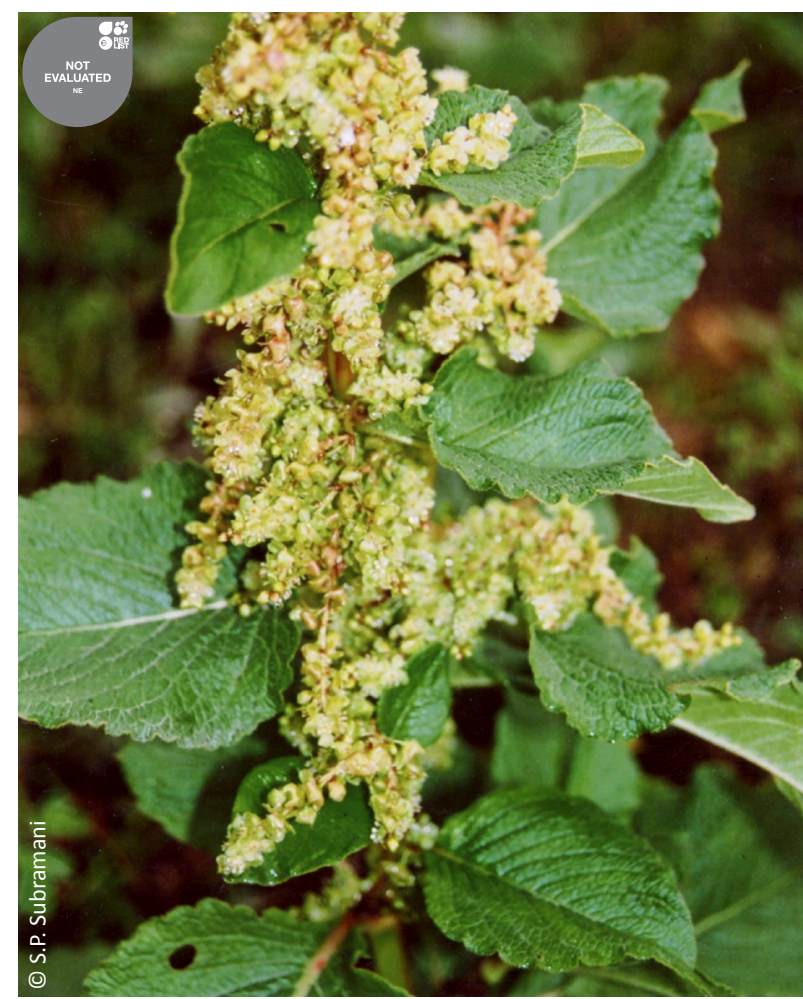

Image 18. Aconogonon rumicifolium (Royle ex Bab.) H.Hara

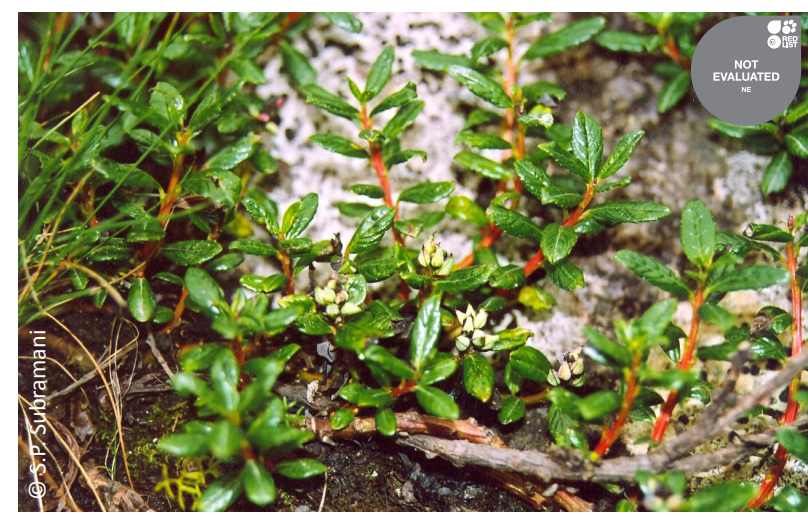

Image 20. Salix flagellaris Hultén

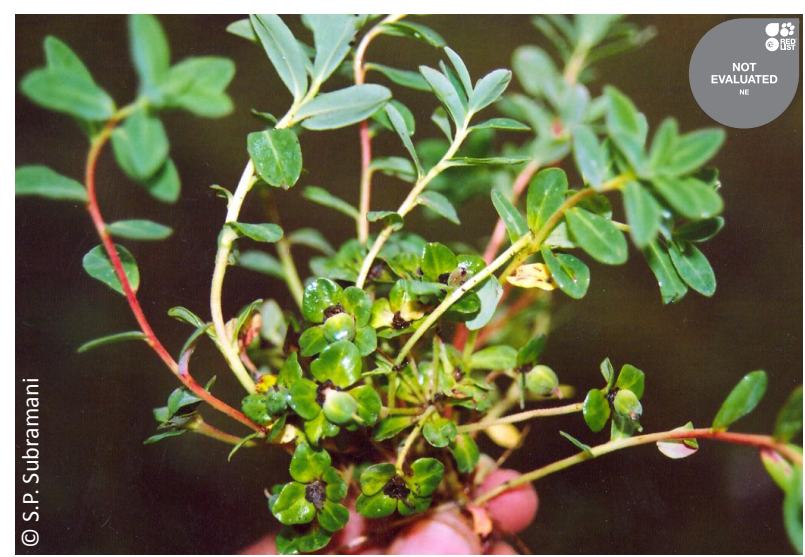

Image 22. Euphorbia stracheyi Boiss.

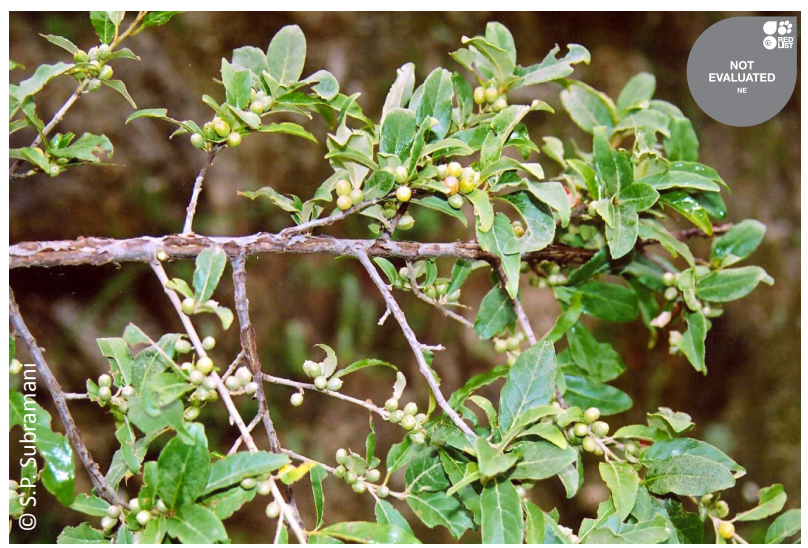

Image 19. Elaeagnus parvifolia Wall. ex Royle

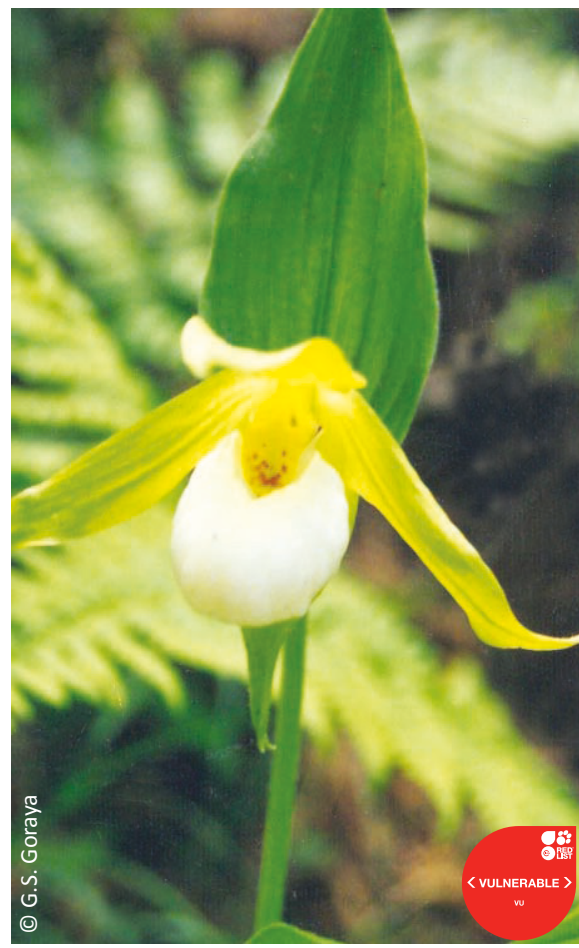

Image 21. Cypripedium cordigerum D. Don 

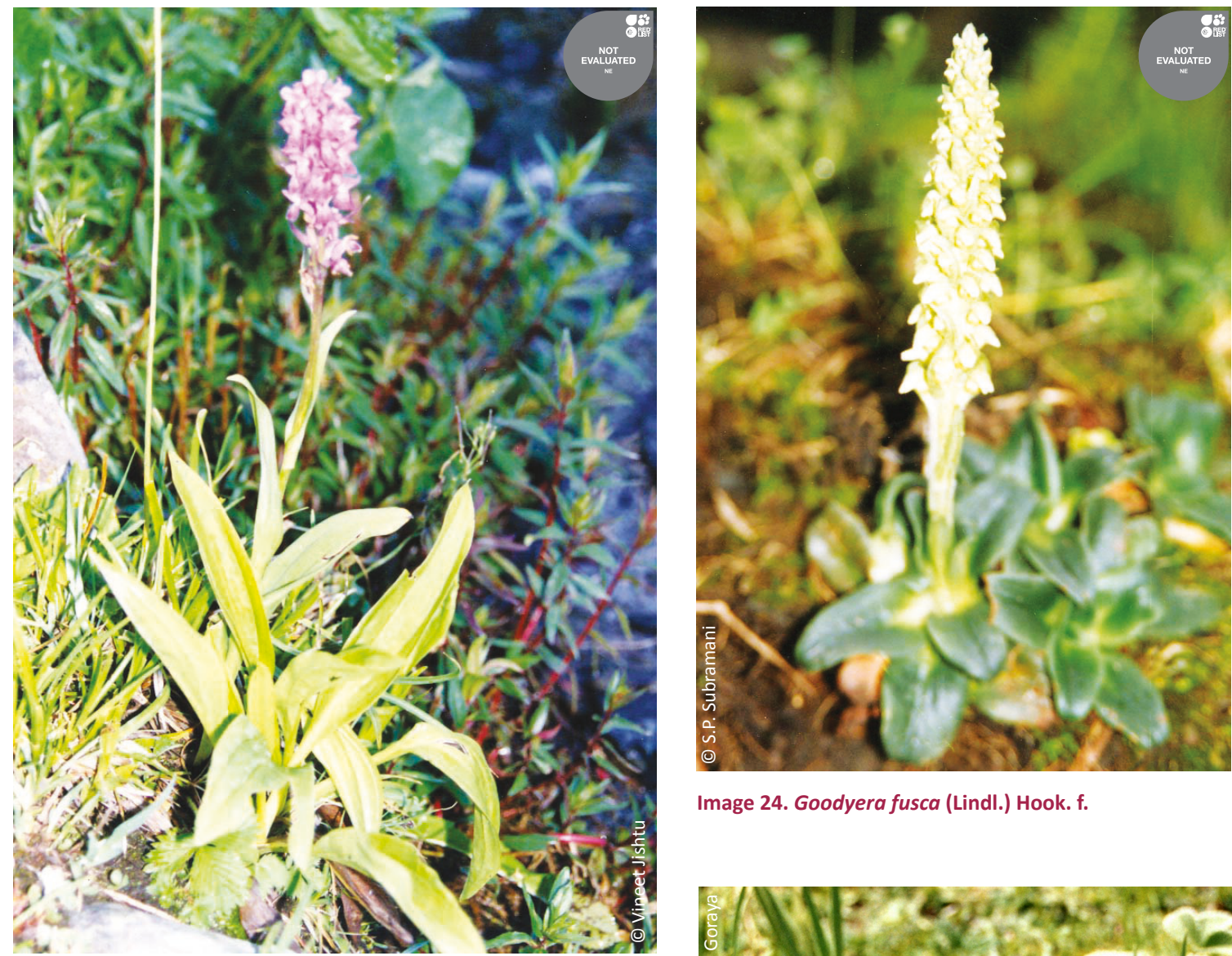

Image 24. Goodyera fusca (Lindl.) Hook. f.

Image 23. Dactylorhiza hatagirea (D.Don) Soo
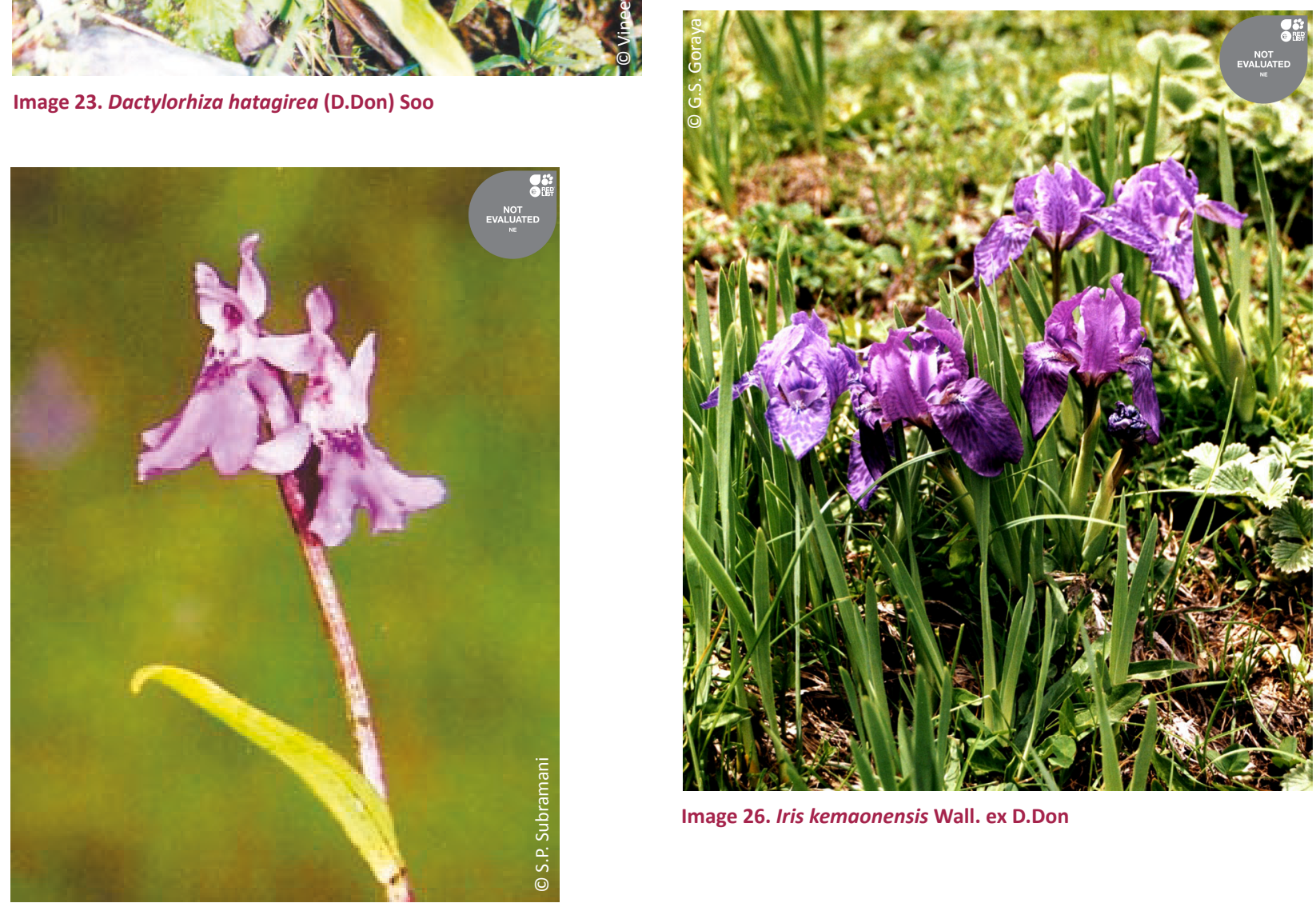

Image 26. Iris kemaonensis Wall. ex D.Don

Image 25. Ponerorchis chusua (D. Don) Soo 

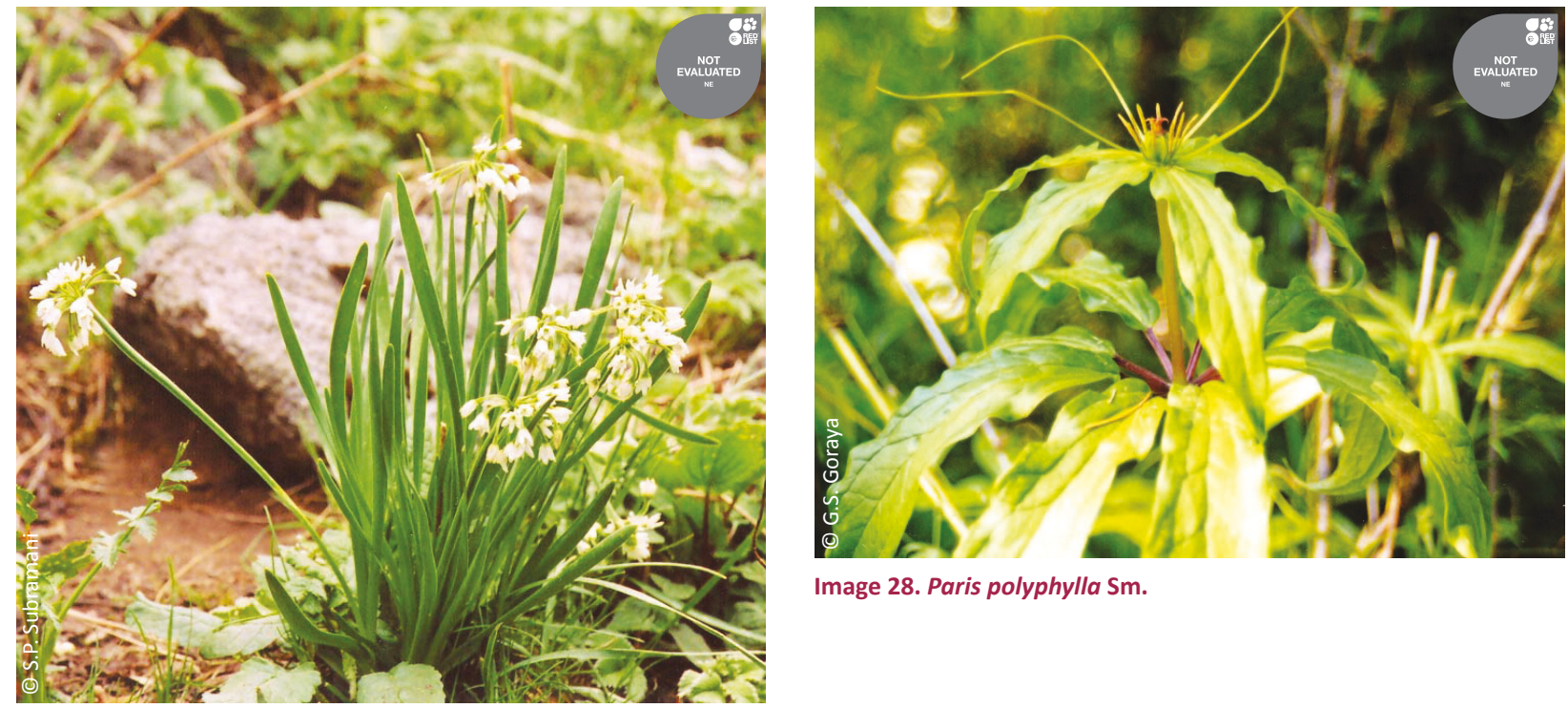

Image 28. Paris polyphylla Sm.

Image 27. Allium humile Kunth
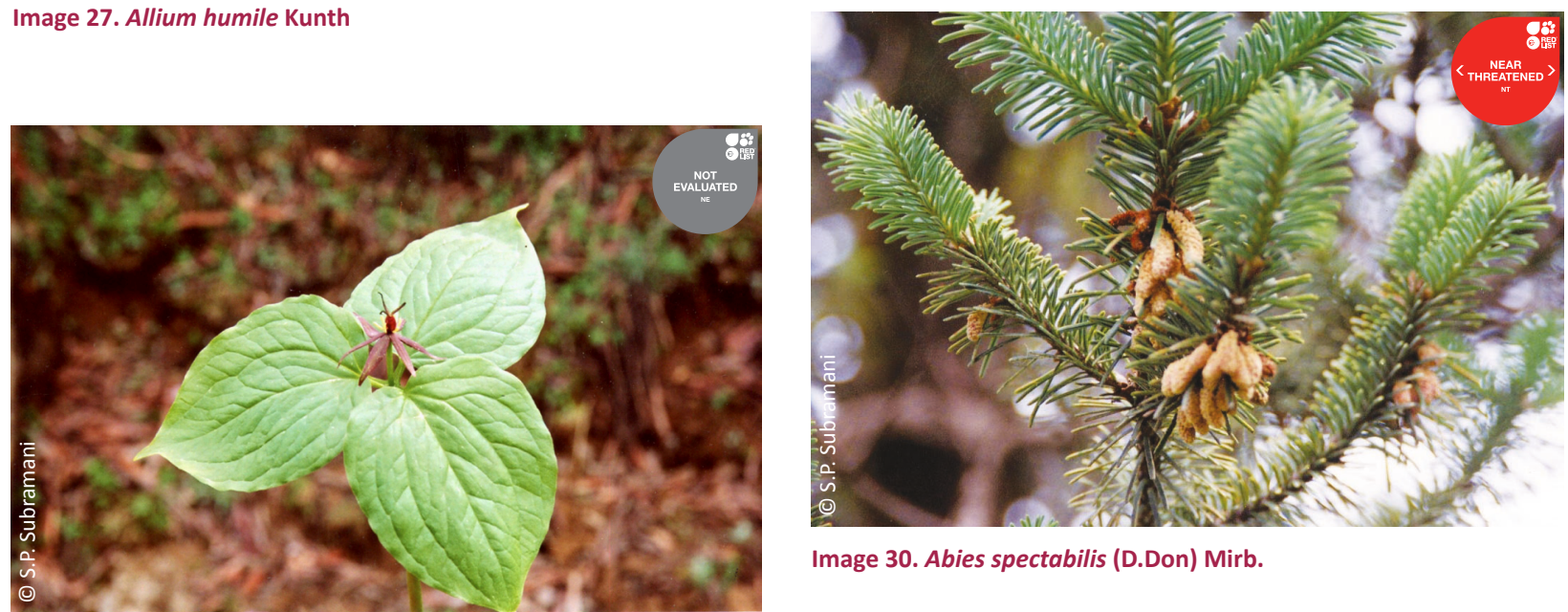

Image 30. Abies spectabilis (D.Don) Mirb.

Image 29. Trillium govanianum Wall. ex D. Don

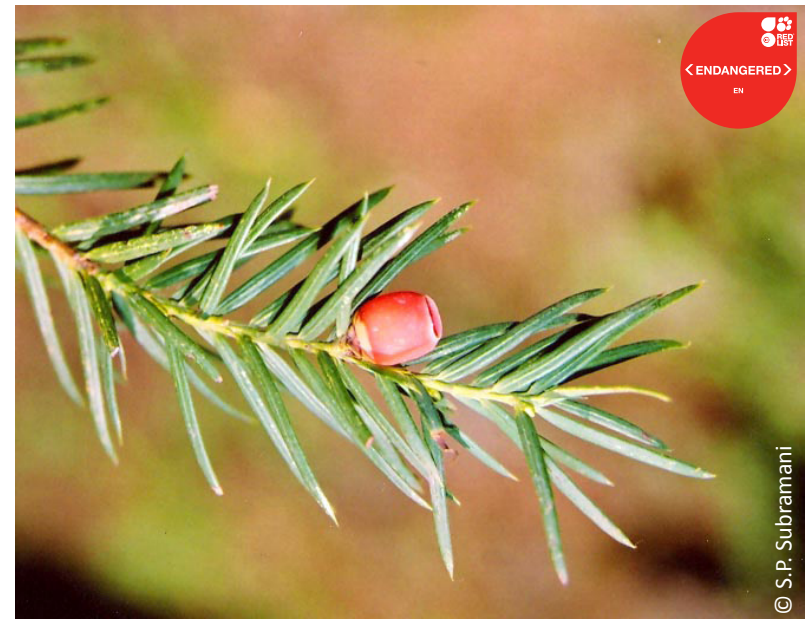

Image 31. Taxus wallichiana Zucc.

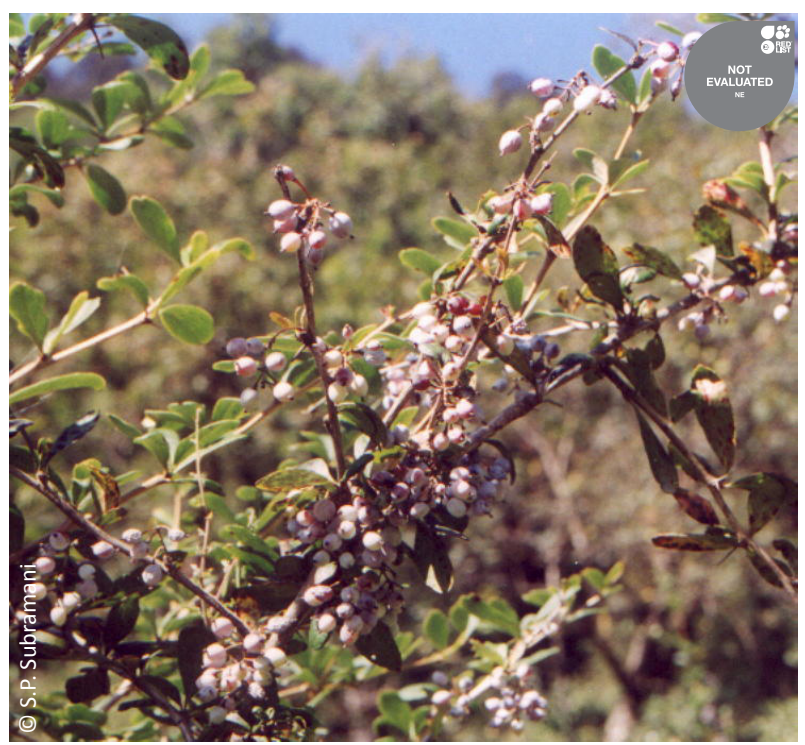

Image 32. Berberis aristata DC. 


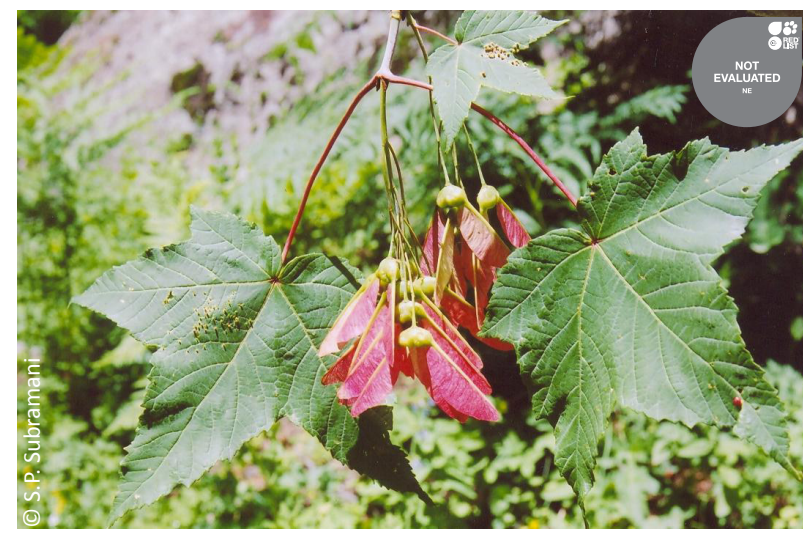

Image 33. Acer caesium Wall. ex Brandis

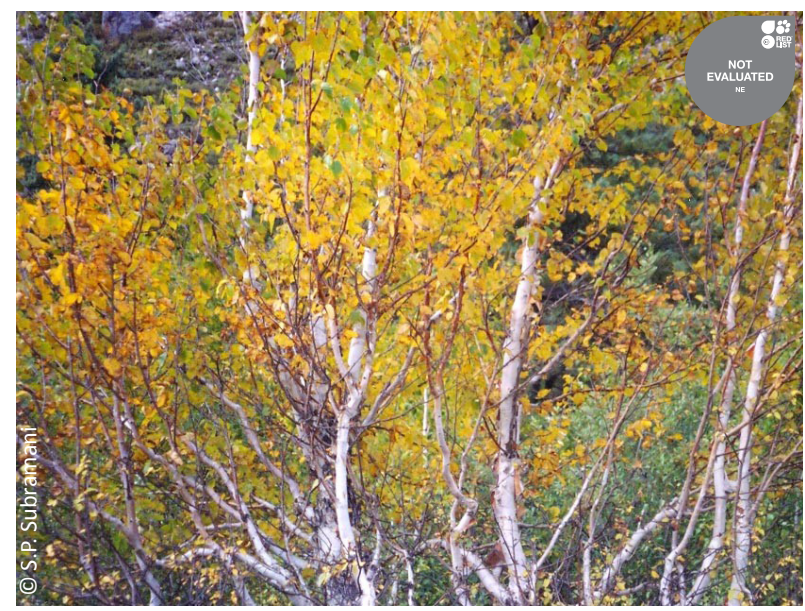

Image 35. Betula utilis D. Don

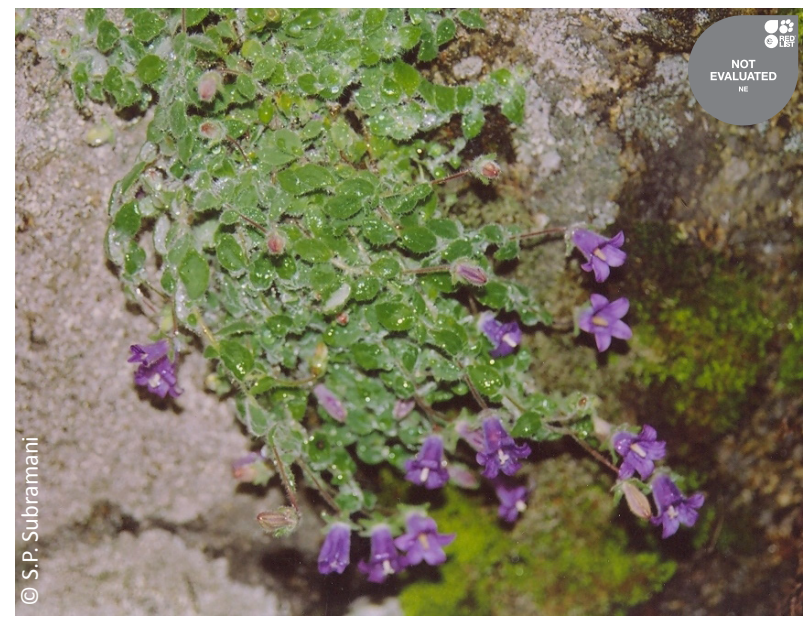

Image 37. Campanula argyrotricha Wall. ex A. DC.

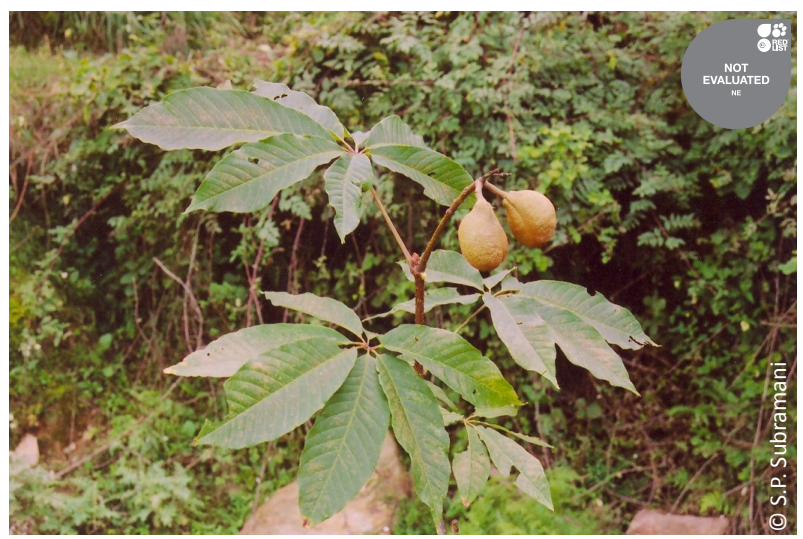

Image 34. Aesculus indica (Wall. ex Cambess.) Hook

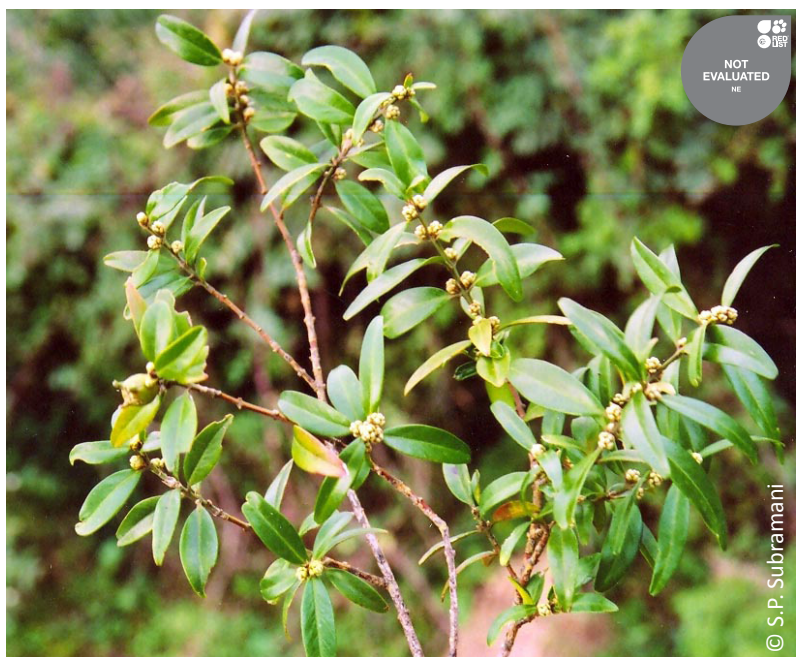

Image 36. Buxus wallichiana Baill.

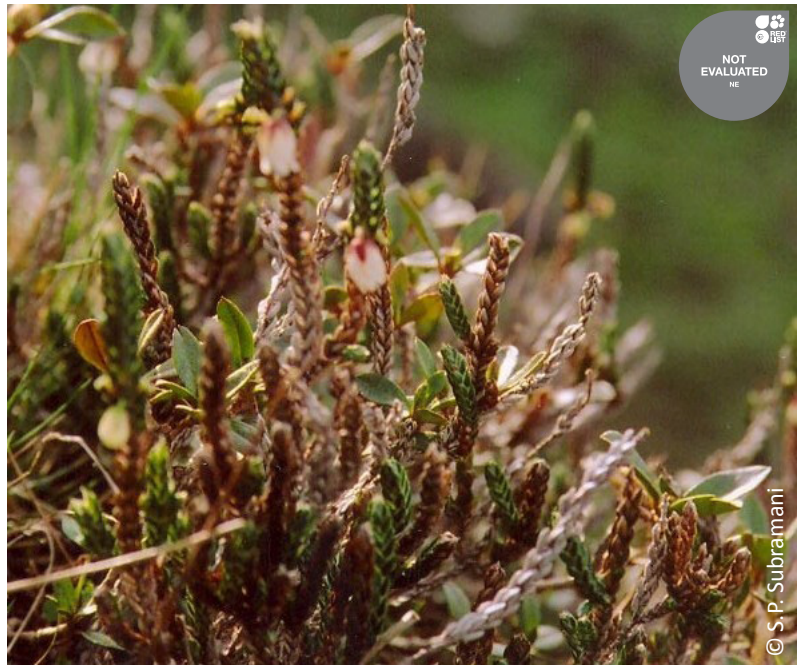

Image 38. Cassiope fastigiata (Wall.) D. Don 

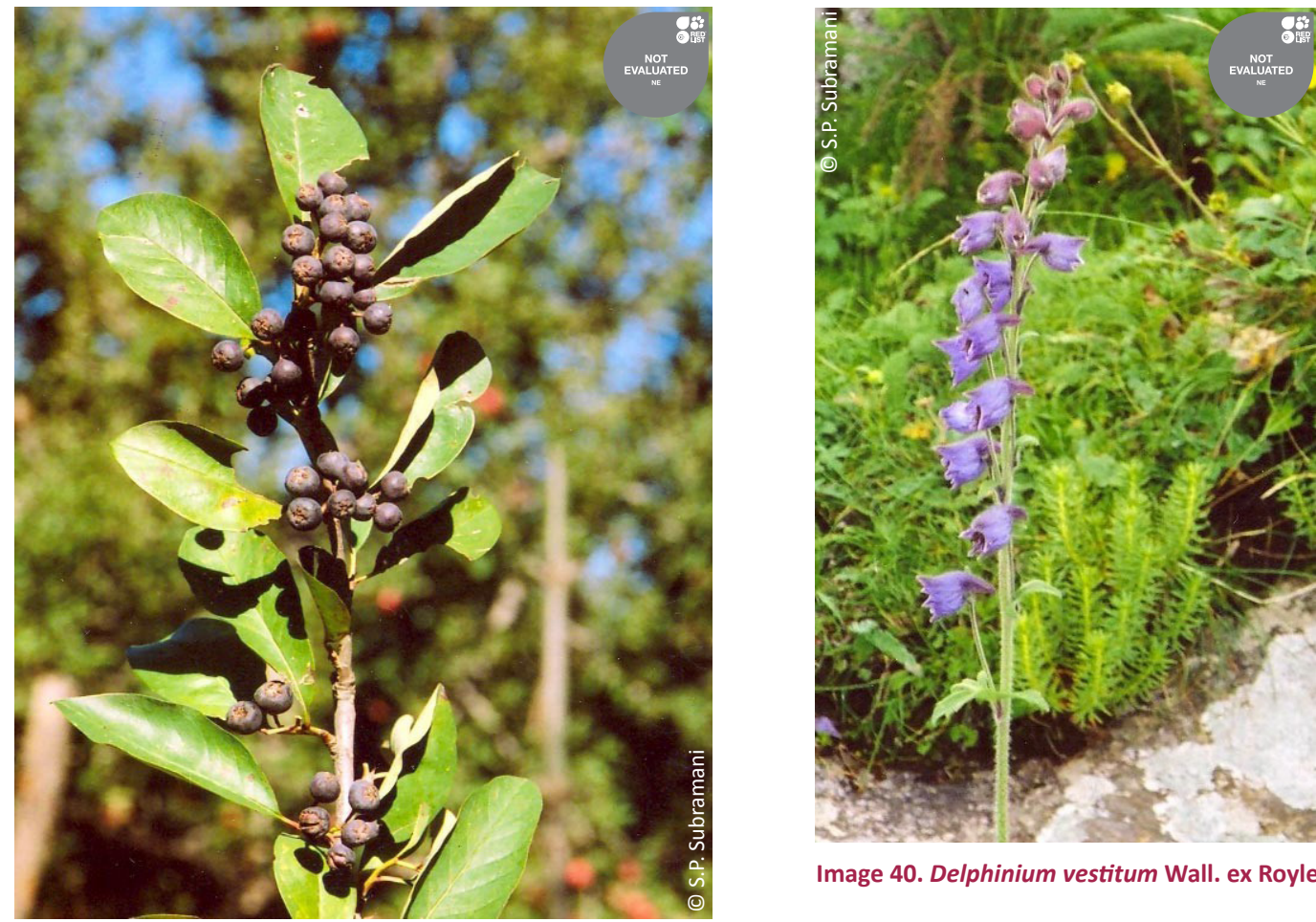

Image 40. Delphinium vestitum Wall. ex Royle

Image 39. Cotoneaster bacillaris Wall. ex Lindl.

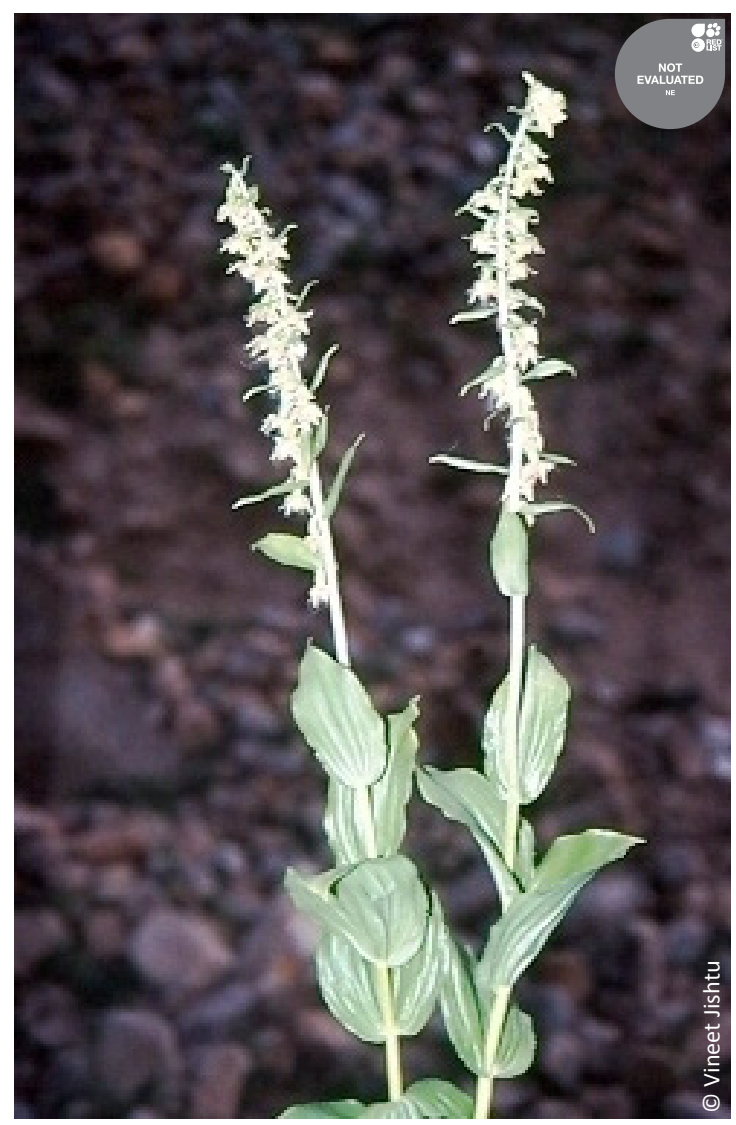

Image 41. Epipactis helleborine (L.) Crantz

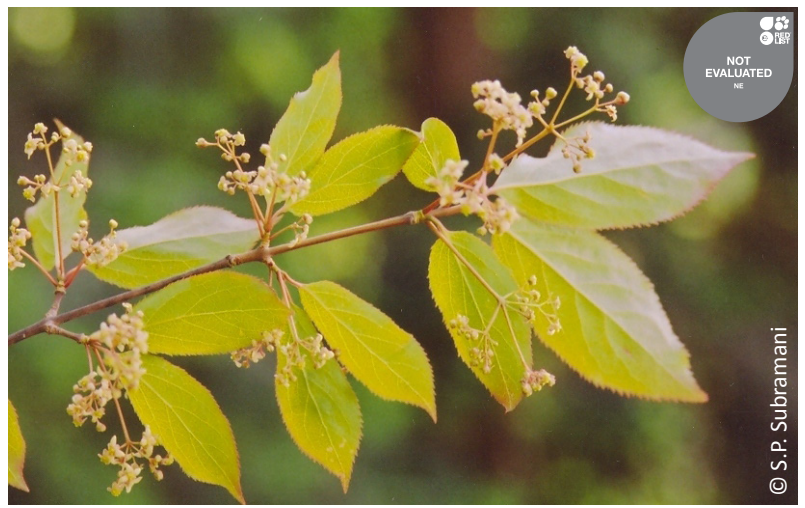

Image 42. Euonymus hamiltonianus Wall.

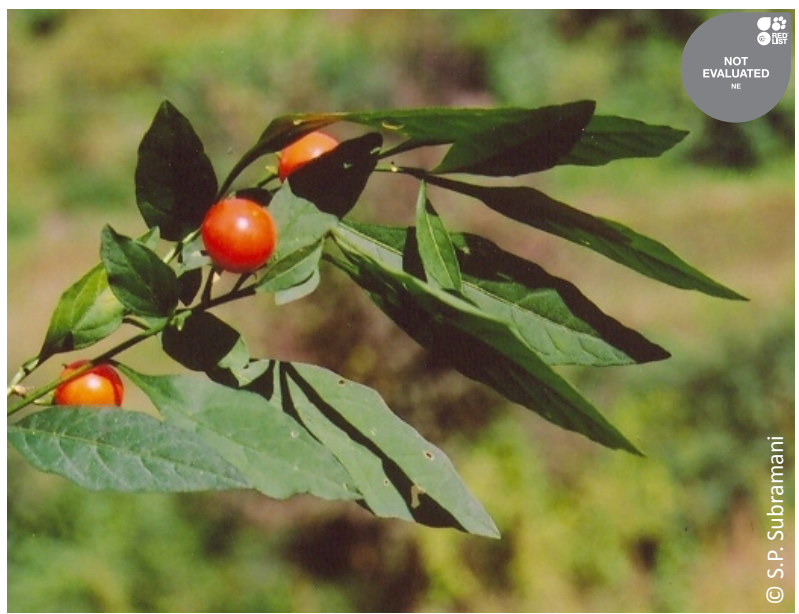

Image 43. Solanum pseudocapsicum L. 


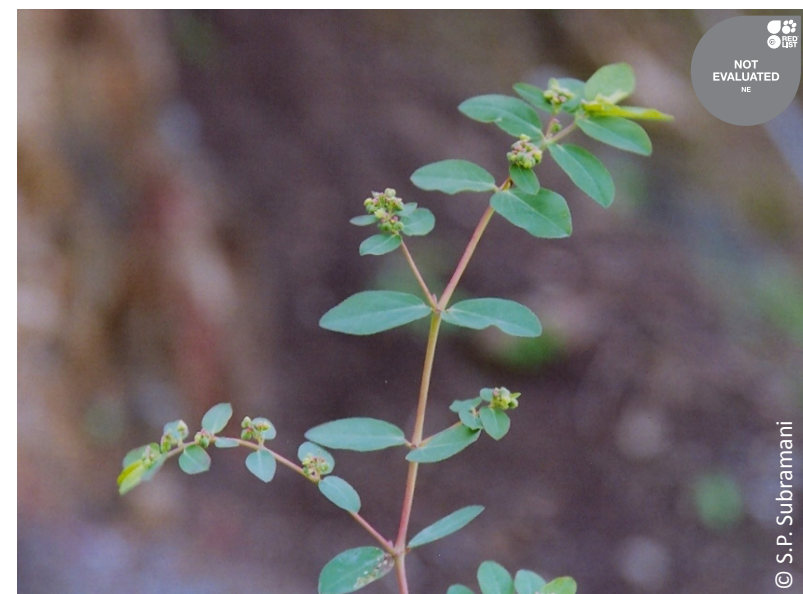

Image 44. Euphorbia parviflora L.

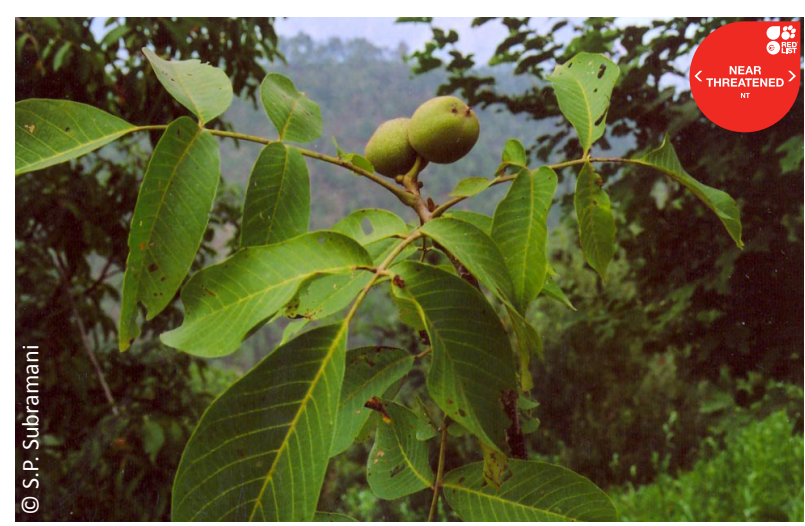

Image 46. Juglans regia L.

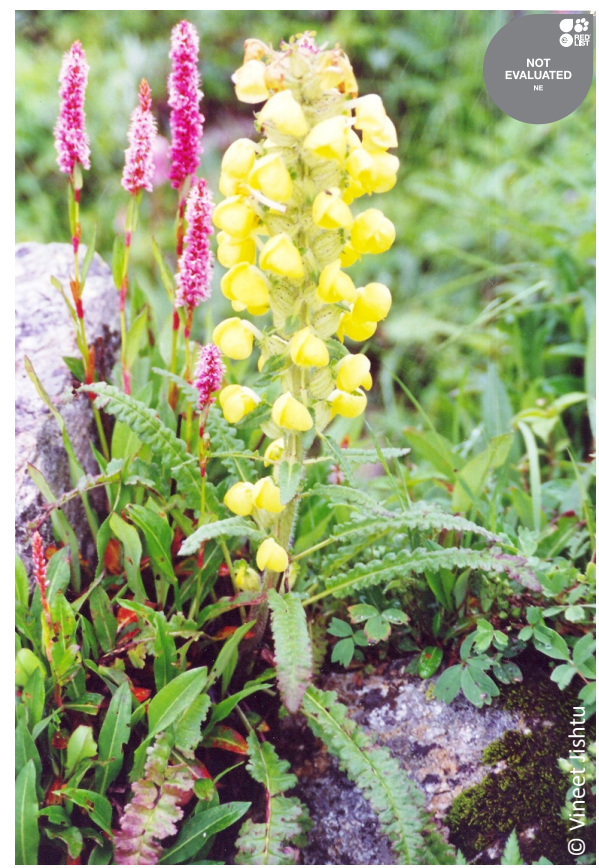

Image 48. Pedicularis bicornuta Klotzsch

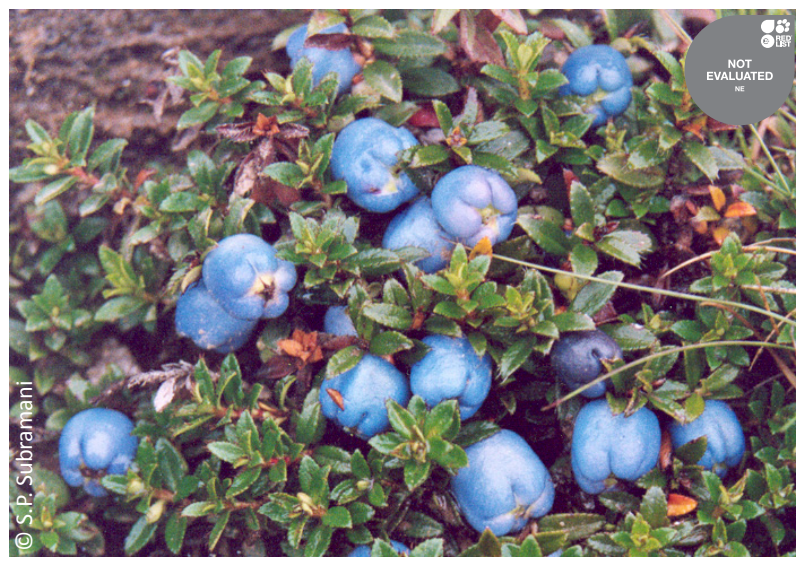

Image 45. Gaultheria trichophylla Royle

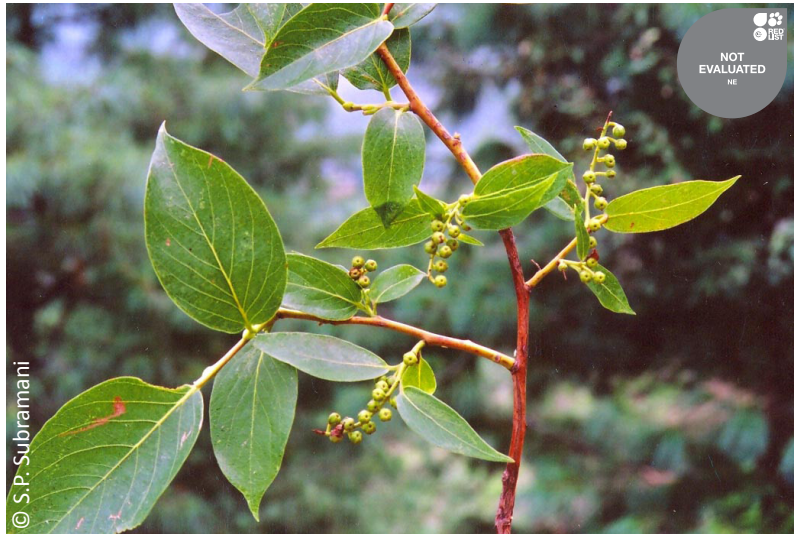

Image 47. Lyonia ovalifolia (Wall.) Drude

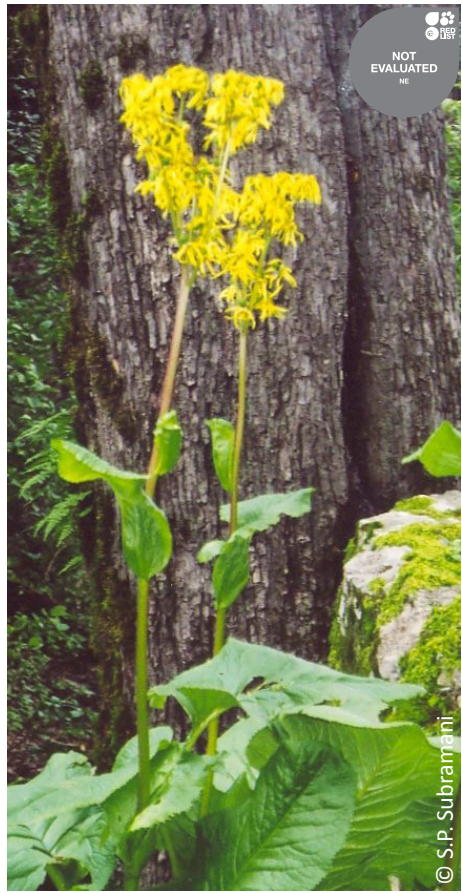

Image 49. Ligularia amplexicaulis DC 


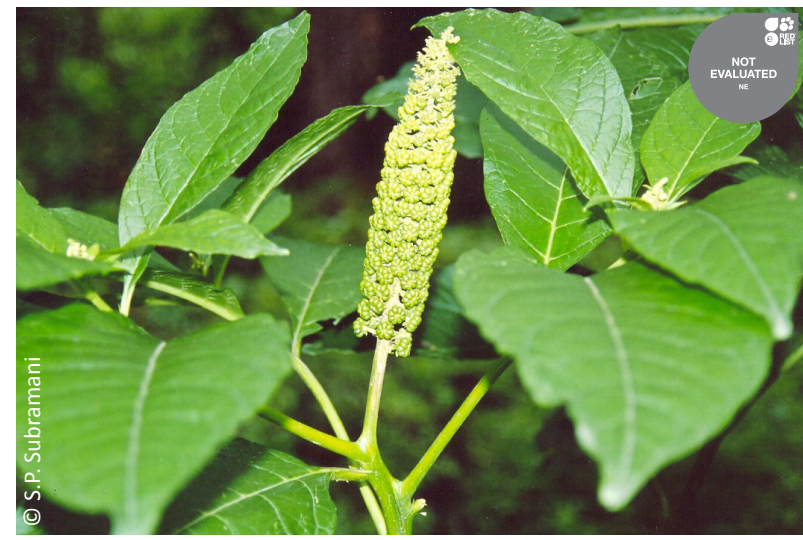

Image 50. Phytolacca acinosa Roxb.

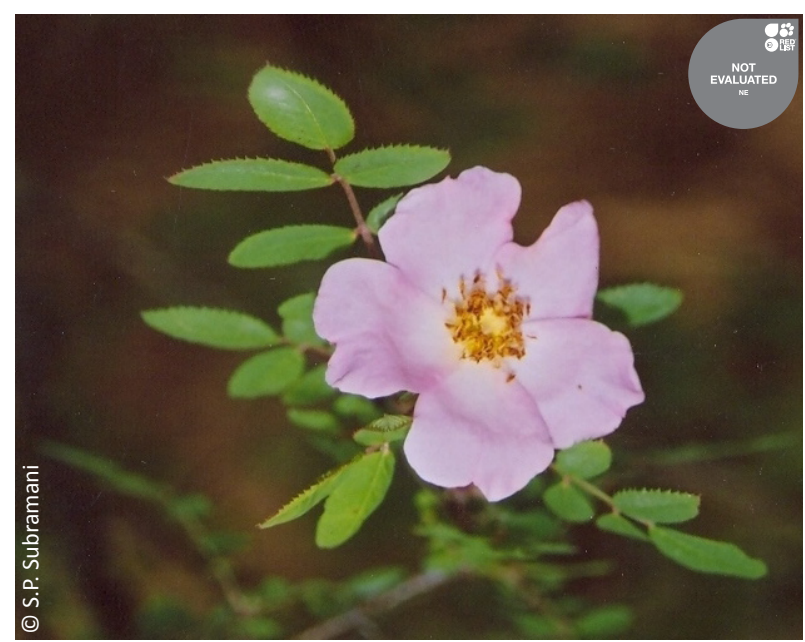

Image 52. Rosa macrophylla Lindl.

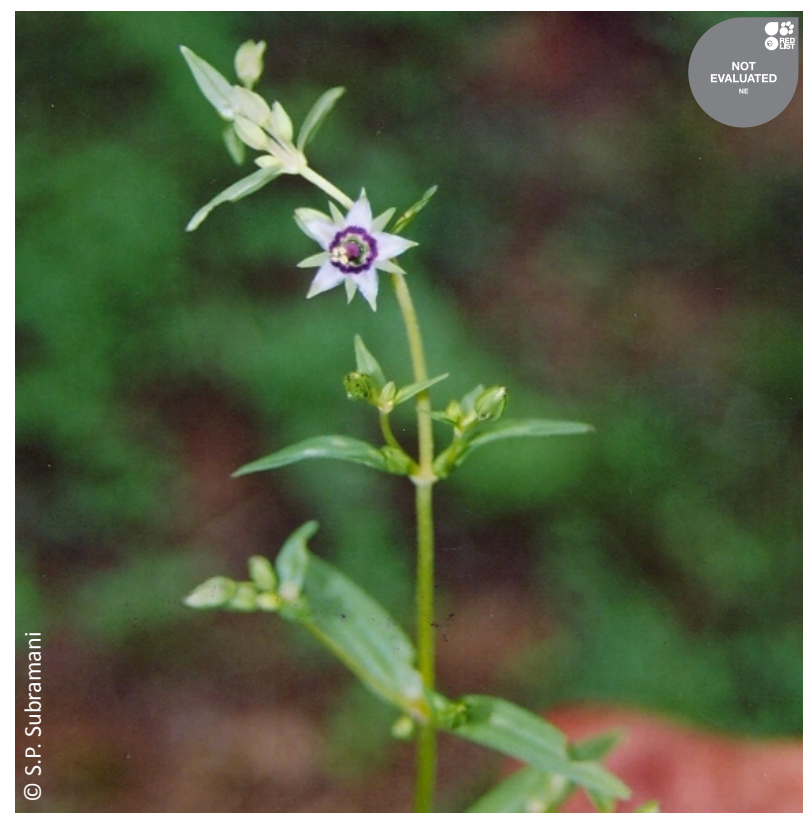

Image 54. Swertia ciliata (D.Don ex G. Don) B.L. Burtt

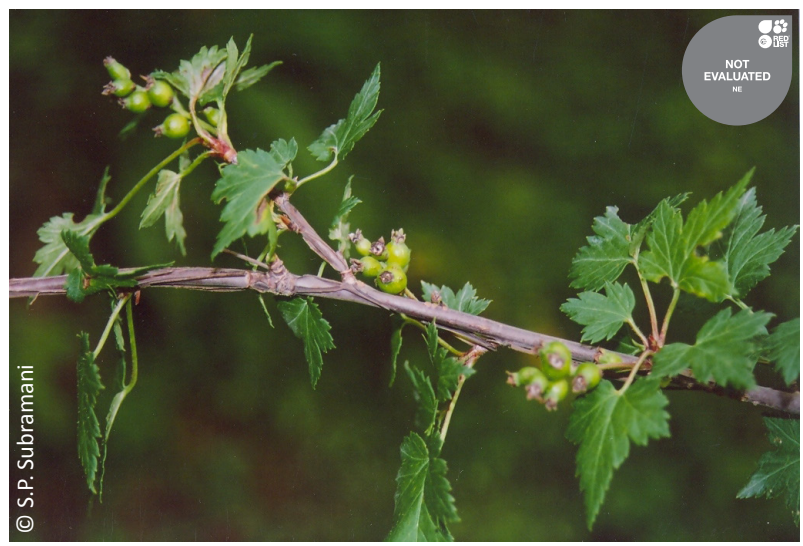

Image 51. Ribes glaciale Wall.

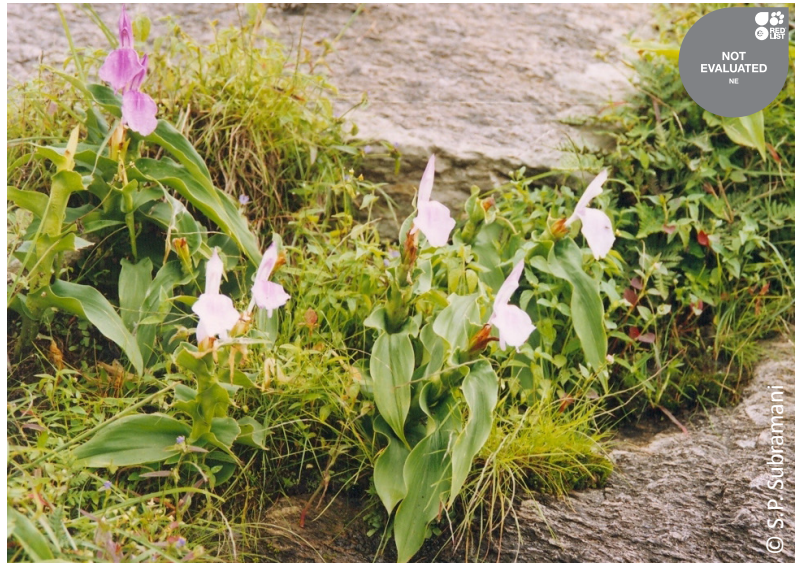

Image 53. Roscoea purpurea Sm.

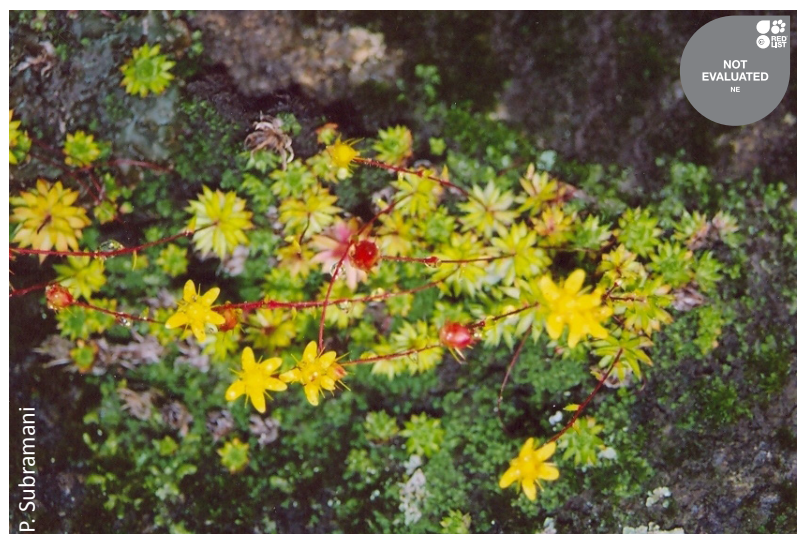

In:age 55. Saxifraga brunonis Wall. ex Ser.

Thomas, P. \& A. Farjon (2011). Taxus wallichiana. The IUCN Red List of Threatened Species. Version 2014.2. <www.iucnredlist.org>. Downloaded on 11 October 2014.

Ved, D.K., G.K. Kinhal, K. Ravikumar, V. Prabhakaran, U. Ghate, R.V. Sankar \& J.H. Indresha (eds.) (2003). Report of the Conservation and Assessment Management Prioritization for the Medicinal Plants of Jammu and Kashmir, Himachal Pradesh and Uttaranchal. FRLHT, Bangalore, 206pp. 